Measurements of Dopamine in Drosophila using Fast-Scan Cyclic Voltammetry

Trisha Lynette Vickrey

Planet Earth

B.S., University of Mississippi, 2005

A Dissertation presented to the Graduate Faculty of the University of Virginia in Candidacy for the Degree of

Doctor of Philosophy

Department of Chemistry

University of Virginia

July 2012

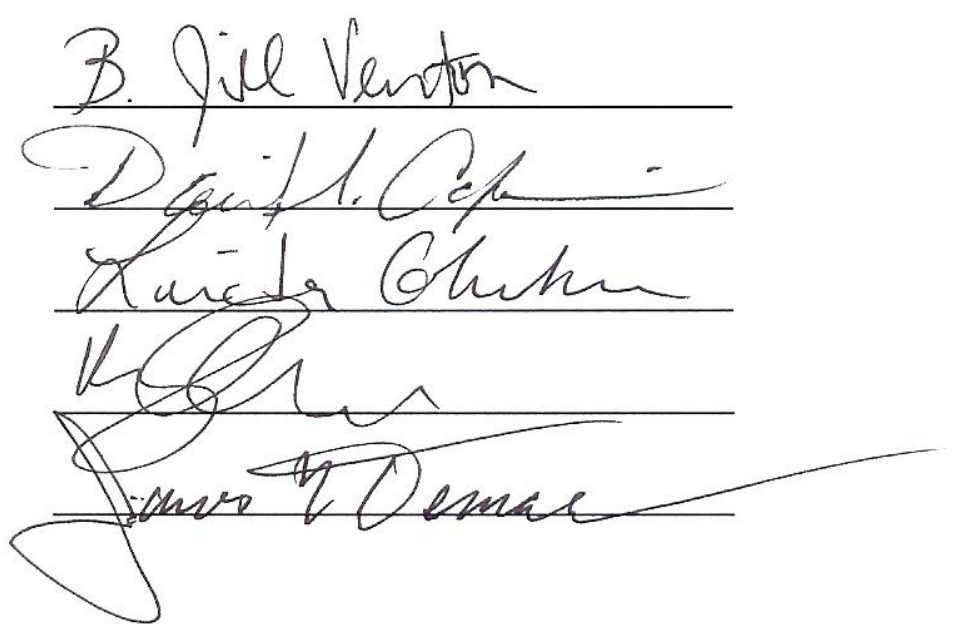




\title{
Measurements of Dopamine in Drosophila using Fast-Scan Cyclic Voltammetry
}

\begin{abstract}
:
Drosophila melanogaster, the fruit fly, is homologous to mammals in primitive neurobiology making it an advantageous model system in which to study the dynamics of dopamine regulation. However, there are few methods for measuring real-time release of dopamine in the intact fly nervous system because the size of the central nervous system (CNS) is so small. My dissertation research overcomes critical instrumentation barriers to develop the first real-time detection method for dopamine release in the fruit fly. Two methods using fast-scan cyclic voltammetry were developed to characterize dopamine homeostasis in an intact Drosophila CNS. In the first method, stimulated release was measured in Drosophila genetically modified to specifically express Channelrhodopsin2 (ChR2), a blue-light activated cation channel, in only dopaminergic neurons. In the second method, a micropipette back-filled with dopamine was implanted 15-20 $\mu \mathrm{m}$ away from a carbon-fiber microelectrode and clearance measured after dopamine was pressure ejected into the CNS. These novel methods open up the fly as a model system for studying basic mechanisms of neurotransmission.
\end{abstract}




\section{Table of Contents}

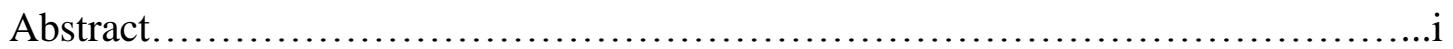

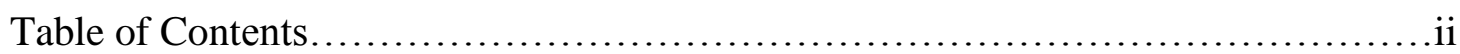

List of Figures...................................................................

List of Equations................................................................

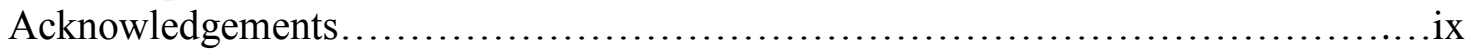

Chapter 1: $\quad$ Introduction.................................................................1

1.1 Fast-Scan Cyclic Voltammetry.................................2

1.1.1 Overview of fast-scan cyclic voltammetry...................2

1.1.2 The FSCV waveform for dopamine detection...................3

1.1.3 Current and the cyclic voltammogram.......................5

1.1.4 Carbon-fiber microelectrodes.............................9

1.1.5 Comparison to other techniques...........................11

1.2 Measuring Dopamine in vivo.................................... 14

1.2.1 Dopamine neurotransmission............................14

1.2.2 FSCV and dopamine detection..........................18

1.2.3 Kinetic modeling........................................20

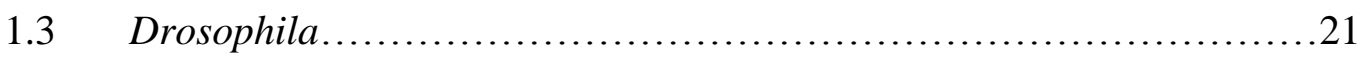

1.3.1 Drosophila as a model..................................21

1.3.2 The UAS-GAL4 system................................23

1.3.3 ChR2 and Drosophila..................................24

1.3.4 Dopamine in the larval ventral nerve cord $\ldots \ldots \ldots \ldots \ldots \ldots \ldots . . .25$

1.3.5 Using FSCV to characterize dopamine neurotransmission in

Drosophila...........................................28

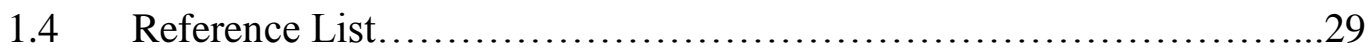




\section{Chapter 2: Detection of endogenous dopamine changes in Drosophila}

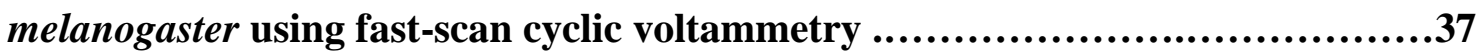

Abstract......................................................... 38

$2.1 \quad$ Introduction.................................................... 39

2.2 Results and Discussion........................................42

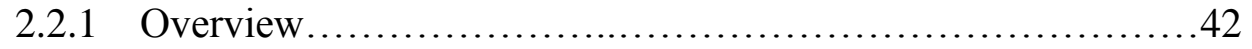

2.2.2 Anatomy of Drosophila dopamine neurons...................42

2.2.3 Measurements of endogenous dopamine evoked by

Channelrhodopsin2 stimulation

2.2.4 Effects of stimulation parameters on evoked dopamine release...48

2.2.5 Characterization of dopamine release using pharmacology......49

2.2.6 Characterization of dopamine uptake using pharmacology......52

2.2.7 Comparison to other techniques..........................55

2.3 Conclusions................................................57

$2.4 \quad$ Experimental............................................... 58

2.4.1 Chemicals.............................................58

2.4.2 Electrochemical Measurements.............................58

2.4.3 Preparation of ventral nerve cords........................60

2.4.4 Immunohistochemistry Experiments........................61

2.5 Reference List

Chapter 3: Drosophila dopamine2-like receptors function as autoreceptor.......67

Abstract..........................................................68

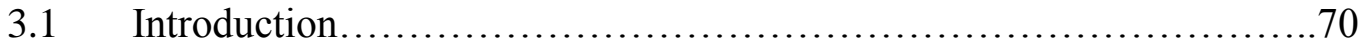




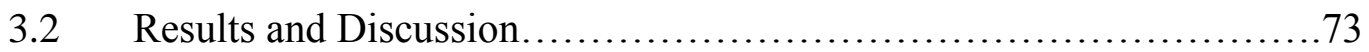

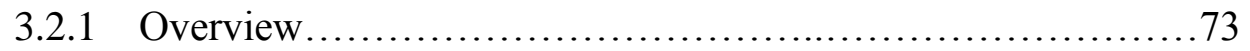

3.2.2 Dopamine agonists decrease evoked dopamine release............74

3.2.3 Dopamine antagonists increase evoked dopamine release.........78

3.2.4 Raclopride increases basal dopamine levels....................81

3.2.5 Interactions of D2 receptors and DAT ......................... 85

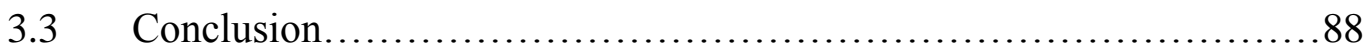

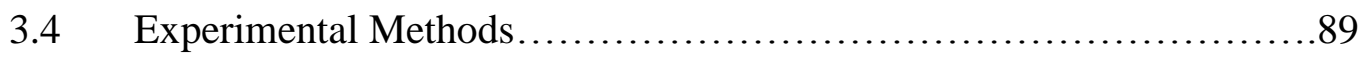

3.4.1 Chemicals......................................................... 89

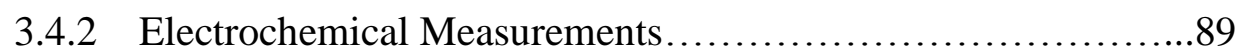

3.4.3 Preparation of Ventral Nerve Cords ..........................90

3.4.4 Statistical Analysis..........................................91

3.4.5 Rat brain slice experiment.................................92

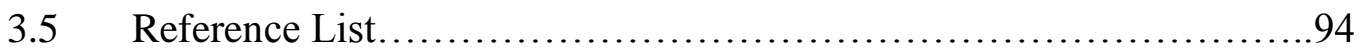

\section{Chapter 4: Voltammetric assessment of dopamine transporter activity in}

Drosphila......................................................................100

Abstract......................................................................

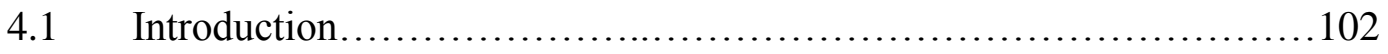

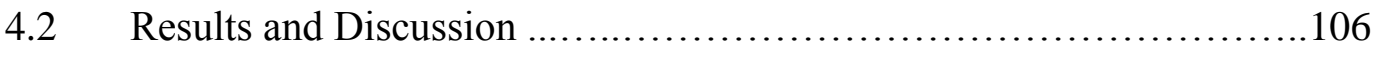

4.2.1 Drosophila uptake via stimulated release......................106

4.2.2 Drosophila uptake via application of exogenous dopamine......108

4.2.3 Drosophila uptake in fumin mutants..........................111

4.2.4 Drosophila uptake in the presence of cocaine..................114 
4.2.5 Uptake in Drosophila mutants...........................116

$4.3 \quad$ Conclusion.................................................... 119

$4.4 \quad$ Experimental Methods........................................ 120

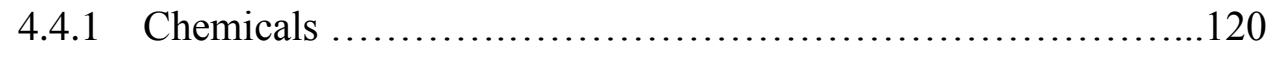

4.4.2 Electrochemical Measurements ...........................120

4.4.3 Preparation of the Drosophila CNS ........................121

4.4.4 Data Analysis........................................ 122

$4.5 \quad$ Reference List.............................................. 124

Chapter 5: Conclusions and future directions..................................128

5.1 Investigating real-time dopamine in Drosophila adults and in disease

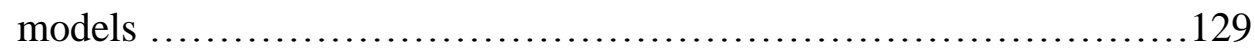

5.2 Investigation of transporters and large scale screening methods........ 132

$5.3 \quad$ Final Remarks............................................. 133

$5.4 \quad$ Reference List............................................. 134 


\section{List of Figures}

1.1 Typical triangular FSCV waveform for dopamine detection...................

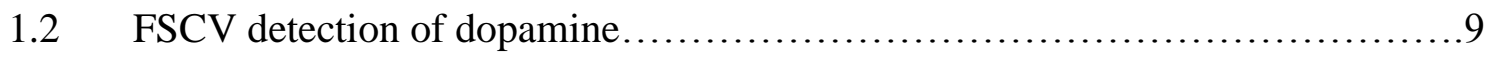

1.3 Scanning electron microscopic image of a cylindrical carbon-fiber

microelectrode...................................................10

1.4 Major components of a neuron.................................... 15

1.5 Dopaminergic Synthesis and Neuron.................................. 17

1.6 UAS-GAL4 system in Drosophila...................................24

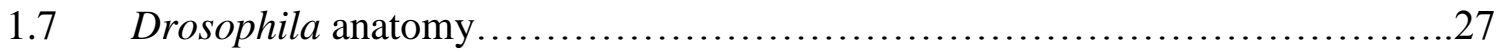

2.1 Fluorescence microscopy of CNS and electrode implantation................43

2.2 Characterization of dopamine signal evoked by ChR2 activation..............45

2.3 Effect of repeated stimulations...................................... 48

2.4 Effect of stimulation duration on dopamine signaling $\ldots \ldots \ldots \ldots \ldots \ldots \ldots \ldots \ldots . \ldots 49$

2.5 Effect of pharmacological agents on evoked dopamine concentration..........51

2.6 Effect of DAT inhibitors on dopamine signaling .........................53

3.1 Effect of D2 agonist bromocriptine on evoked dopamine.................. 75

3.2 Effect of D2 agonist quinpirole.................................... 77

3.3 Effects of the DAT inhibitor with D2R agonists and antagonists..............79

3.4 Effect of raclopride................................................ 82

3.5 Effects of the DAT inhibitor with D2R agonists and antagonists.............86

4.1 Modeling DAT with stimulated release................................. 108

4.2 Application of exogenous dopamine in a Drosophila CNS ..................110

4.3 Application of exogenous dopamine in a fmn mutant......................113 
4.4 Application of exogenous dopamine in the presence of cocaine...............115

4.5 Signal decay in Drosophila mutants...................................117

4.6 Immobilization and electrode implantation in adult Drosophila..............131 


\section{List of Equations}

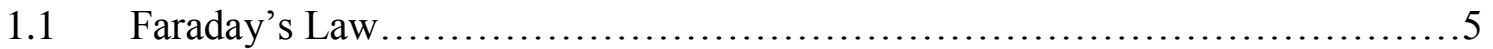

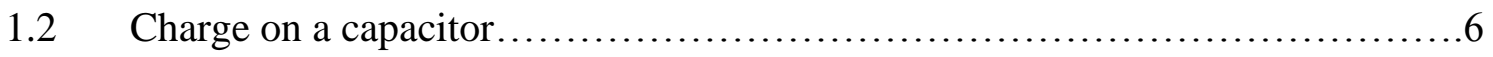

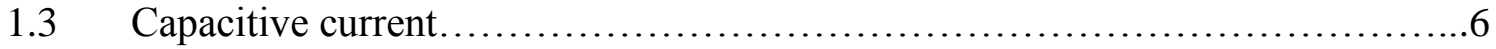

$1.4 \quad$ Electrode capacitance...................................................

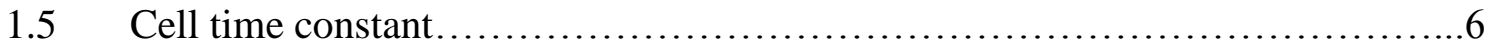

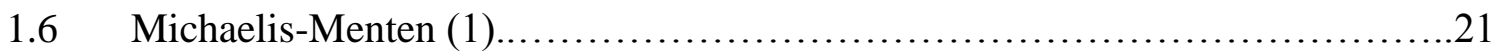

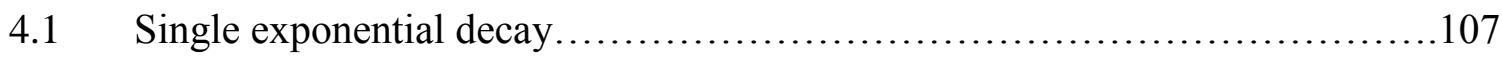

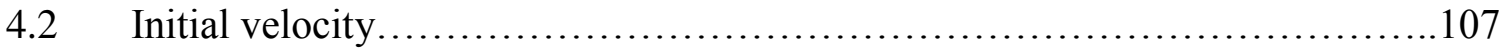

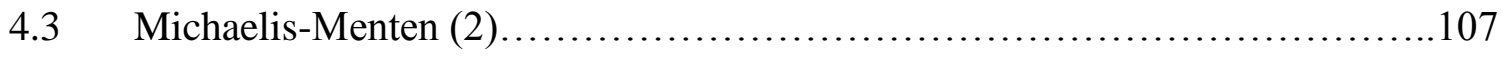

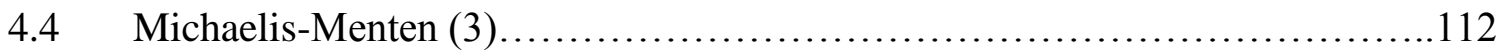




\section{Acknowledgements}

This thesis is dedicated to my dad. Although we didn't have much time together, you taught me the things that led me here. You taught me to appreciate the beauty of the natural world. We touched the sequoias in the redwood forest, stood before the Great Salt Lake, watched salmon spawn in the Sierra Nevadas, and spent countless days inspecting tide pools in Oregon. These experiences made curious about life, made me want to discover how things work, and made me want to be a scientist. You taught me that hard work and perseverance makes you good at something. Every day in the spring you picked me up from school and we played catch...even when I didn't want to. All of that hard work and consistency showed me what it takes to be really good at something. I never would have made it through graduate school without such an important lesson. You taught me to root for the underdog, the self-made man, in politics and in life. I remember once you gave all of our softball equipment to a girl on my team whose parents couldn't afford to get her any of her own. Another time, when a co-worker's wife was diagnosed with cancer, you gave him an entire week's paycheck to help them make ends meet. Witnessing your selflessness and compassion for those who were struggling shaped my life more than anything else. I would neither have arrived at this point, nor be embarking on my future path without empathy, and a desire to help others. Thank you for being such a great dad. I wish that you were still around to see what I've accomplished. 


\section{Chapter 1}

\section{Introduction}

If the world were merely seductive, that would be easy. If it were merely challenging, that would be no problem. But I arise in the morning torn between a desire to improve the world and a desire to enjoy the world. This makes it hard to plan the day.

$\sim$ E.B. White 


\section{Chapter 1: Introduction 1.1: Fast-Scan Cyclic Voltammetry}

\subsubsection{Overview of fast-scan cyclic voltammetry:}

The overall goal of this work was to develop a method to measure changes in dopamine in an intact Drosophila larval CNS using fast-scan cyclic voltammetry at carbon-fiber microelectrodes. Therefore, the introduction to this thesis will focus on the principles of fast-scan cyclic voltammetry, dopamine neurotransmission, detection of dopamine, and the neurobiology of Drosophila.

Fast-scan cyclic voltammetry (FSCV) is an electrochemical detection method that has been extensively used to detect small, electroactive molecules, such as monoamine neurotransmitters, in vivo as well as in situ (1-11). In fast-scan cyclic voltammetry (> 100 $\mathrm{V} / \mathrm{s}$ ), the potential at the working electrode is linearly swept as a function of time from a starting potential to a final, holding potential, and then swept back from the final potential to the starting potential (Figure 1.1). Typically during the sweep, the analyte of interest is oxidized, releasing electrons, and is subsequently reduced, gaining electrons. The movement of electrons during these reactions produces a current that is measured at the working electrode, which is relative to the reference electrode. The current is proportional to the number of molecules changing redox state. These redox events often occur at unique potentials for a given analyte, and can be measured on a millisecond timescale, which is necessary to evaluate neurotransmitter events. The working electrode used in FSCV is typically made from a carbon fiber (12), which is small in diameter (single-digit to tens of micrometers), and causes minimal damage to tissue (13). Therefore, FSCV offers chemical identification, and high spatial and temporal resolution. In addition, 


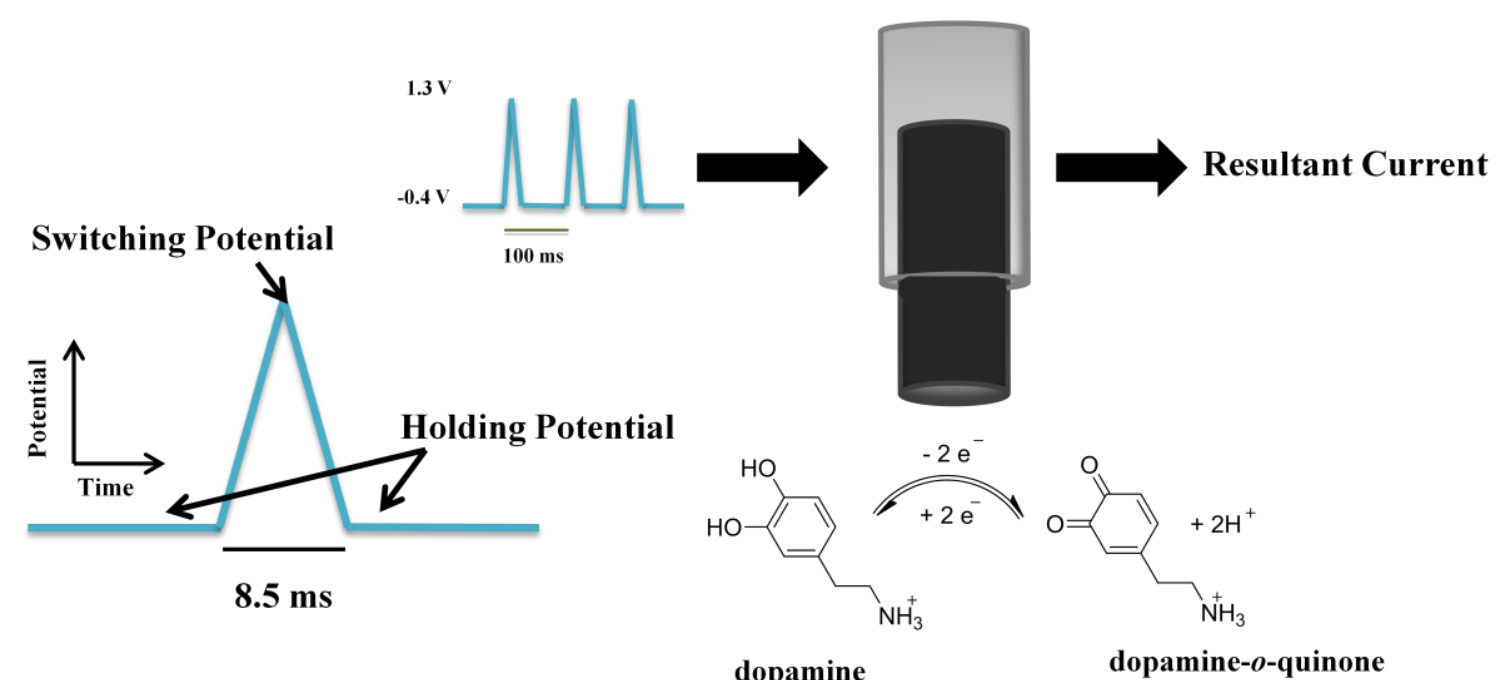

Figure 1.1: Typical triangular FSCV waveform for dopamine detection. The applied potential is ramped up from the holding potential $(-0.4 \mathrm{~V})$ to the switching potential $(1.3 \mathrm{~V})$ and then ramped back down to the holding potential. Each scan is repeated every $100 \mathrm{~ms}$. (inset) at a rate of $400 \mathrm{~V} / \mathrm{s}$. On the forward, or anodic, scan, dopamine is oxidized to form dopamine- $o$-quinone. On the reverse, or cathodic, scan, dopamine- $o$-quinone is reduced back to form dopamine. The movement of electrons from these redox reactions produces time-resolved peaks of current that are measured at the electrode.

FSCV has a limit of detection in the range of tens of nanomolar (nM) for catecholamine neurotransmitters, such as dopamine (14). These characteristics make FSCV at carbon fiber microelectrodes an adequate method for in vivo and in situ detection of dopamine.

\subsection{2: The FSCV waveform for dopamine detection}

With fast-scan cyclic voltammetry (FSCV), a triangular waveform is intermittently applied to an electrode (Figure 1.1). The electrode is kept at a holding potential for a relatively long time to ensure that analytes have sufficient time to absorb onto the carbon-fiber electrode surface (15). Once the triangular waveform is applied, the potential is increased linearly to the switching potential and decreased linearly back to the holding potential at a fast rate of several hundred volts per second. This cycle is repeated at regular intervals sufficient to detect the analyte of interest. For a reversible redox 
process, during the forward, or anodic, part of the scan, the analyte is oxidized and during the back, or cathodic, part of the scan, the analyte is reduced back to its original form. For detection of dopamine using FSCV at a carbon-fiber microelectrode ( $v s$, $\mathrm{Ag} / \mathrm{AgCl})$, the holding potential is typically $-0.4 \mathrm{~V}(15)$. Dopamine is positive at a physiological $\mathrm{pH}$; therefore, holding the electrode at a sufficiently negative potential creates an electrostatic attraction between dopamine and the electrode surface. Potentials lower than $-0.4 \mathrm{~V}$ typically are not used due to the possible reduction of other species, including oxygen (16), and instabilities in background current (17). The longer the time spent at the holding potential, the more time dopamine has to absorb onto the electrode surface (17). However, the longer the time spent at the holding potential, the slower the temporal resolution. The switching potential for dopamine detection is $1.3 \mathrm{~V}$. Scanning to a 1.3 V switching potential increases the possible adsorption sites at the carbon-fiber microelectrode, and when coupled with a $-0.4 \mathrm{~V}$ holding potential, increases the sensitivity of the electrode (17). The switching potential must be kept at or below $1.5 \mathrm{~V}$ to prevent the electrolysis of water.

Once the potential applied to the electrode during the anodic portion of the sweep reaches $0.25 \mathrm{~V}$, dopamine begins to be oxidized into dopamine-o-quinone. The current produced from oxidation reaches a maximum at $0.6 \mathrm{~V}$. During the cathodic portion of the sweep, dopamine-o-quinone is reduced back to dopamine, producing a maximum current at $-0.2 \mathrm{~V}(15)$. Typically the scan rate for dopamine detection is $400 \mathrm{~V} / \mathrm{s}(17,18)$. Thus, the time that it takes to complete the sweep is $8.5 \mathrm{~ms}$ with the sweep repeated every 100 ms. Dopamine neurotransmission occurs on the timescale of $\mathrm{ms}$ to $\mathrm{s}$; therefore, FSCV is a 
suitable technique for measuring neurotransmission (19). Variation of the scan rate, switching and holding potentials and frequency affect selectivity, temporal resolution, and sensitivity $(18,20)$.

\subsection{3: Current and the cyclic voltammogram}

Application of potential to the electrode results in both faradic and non-faradic current. Faradic current is produced during the transfer of electrons between the analyte and electrode that occur during redox reactions. Faradic current is proportional to the number of moles that react; therefore, concentration can be calculated (Equation 1). In FSCV, faradic current is typically produced in the presence of the analyte of interest or electroactive interferents. However, at carbon-fiber microelectrodes, some faradic current is also produced from the oxidation and reduction of functional groups on the surface of the electrode (21).

$\mathrm{Q}=\mathrm{nF} \Delta \mathrm{N}$

\section{Equation 1.1}

$\mathrm{Q}=$ the charge passed across the electrode

$\mathrm{n}=$ the number of electrons per molecule transferred

$\mathrm{F}=$ Faraday's constant $(96,485 \mathrm{C} / \mathrm{mol})$

$\Delta \mathrm{N}=$ number of moles that react

Non-faradic current is produced due to double layer charging (Figure 1.2A). As potential is applied to the electrode, a charge imbalance is created at the electrodesolution interface, which causes a rearrangement of charged species in the solution near the electrode. The electrode-surface interface can be modeled by a capacitor (Equation 1.2) (22). When potential, E, is applied to the electrode, charged species in solution rearrange so that $q$ satisfies Equation 1.2. The current produced during this rearrangement 
of charged species is called charging current. The charging current is proportional to the capacitance at the electrode and increases linearly with scan rate (Equation 1.3) (22).

Thus, the large scan rates used in FSCV create a large charging current. The capacitance of the electrode, however, is proportional to the area of the electrode (Equation 1.4) (23); therefore, the charging current at carbon-fiber microelectrodes, which have small surface areas, decays rapidly (Equation 1.5) (24).

$\mathrm{C}=\frac{q}{\mathrm{E}}$

Equation 1.2

$q=$ charge stored on the capacitor

$\mathrm{E}=$ the potential across the capacitor

$\mathrm{C}=$ capacitance

$\mathrm{i}_{\mathrm{c}}=v \mathrm{C}$

Equation 1.3

$\mathrm{i}_{\mathrm{c}}=$ charging current

$v=$ scan rate

$\mathrm{C}=\frac{\mathrm{A} \varepsilon_{0}}{\mathrm{~d}}$

Equation 1.4

$\mathrm{A}=$ electrode area

$\varepsilon_{0}=$ dielectric constant

$\mathrm{d}=$ distance between electrode and double-layer

$\tau=\mathrm{RC}$

Equation 1.5

$\tau=$ cell time constant

$\mathrm{R}=$ resistance of the solution

Together, the faradaic current from the oxidation of the functional groups at the surface of the carbon-fiber microelectrode and the non-faradaic current from double-layer charging produce a large background current that is stable over time (90 s). Typically in 
FSCV, the background current is large in comparison to the current produced from analyte detection, and must be subtracted. Due to background subtraction, FSCV is best for measuring quick changes in analyte, such as those associated with dopamine neurotransmission, and is not typically used to measure the baseline concentration of analytes. In order to measure dopamine, the background current from a scan without dopamine is subtracted from a scan of the faradaic current produced in the presence of dopamine (Figure 1.2A). The resulting cyclic voltammogram (Figure 1.2B) for dopamine has an oxidation peak at approximately $0.6 \mathrm{~V}$ and a reduction peak at $-0.2 \mathrm{~V}$. The dopamine signal was produced by stimulating dopamine release from an intact, Drosophila central nervous system.

The background-subtracted cyclic voltammogram provides chemical identity for dopamine, but temporal information is also available. Current, voltage and time can be visualized simultaneously on a color plot (Figure 1.2C). Applied potential is on the yaxis. The y-axis is the holding potential, where the waveform begins, the middle is the switching potential, and the bottom is the holding potential, where the waveform ends. Time is on the $\mathrm{x}$-axis. Baseline data is collected for $30 \mathrm{~s}$ followed by application of dopamine (red line). Color is used to represent changes in current. Green represents increases in current produced from the oxidation of dopamine to dopamine-o-quinone, and blue represents decreases in current produced from the reduction of dopamine-oquinone to dopamine. The vertical white line runs through the time point at which maximal dopamine oxidation occurs, and is the time point that for the cyclic voltammogram in Figure 1.2B. 


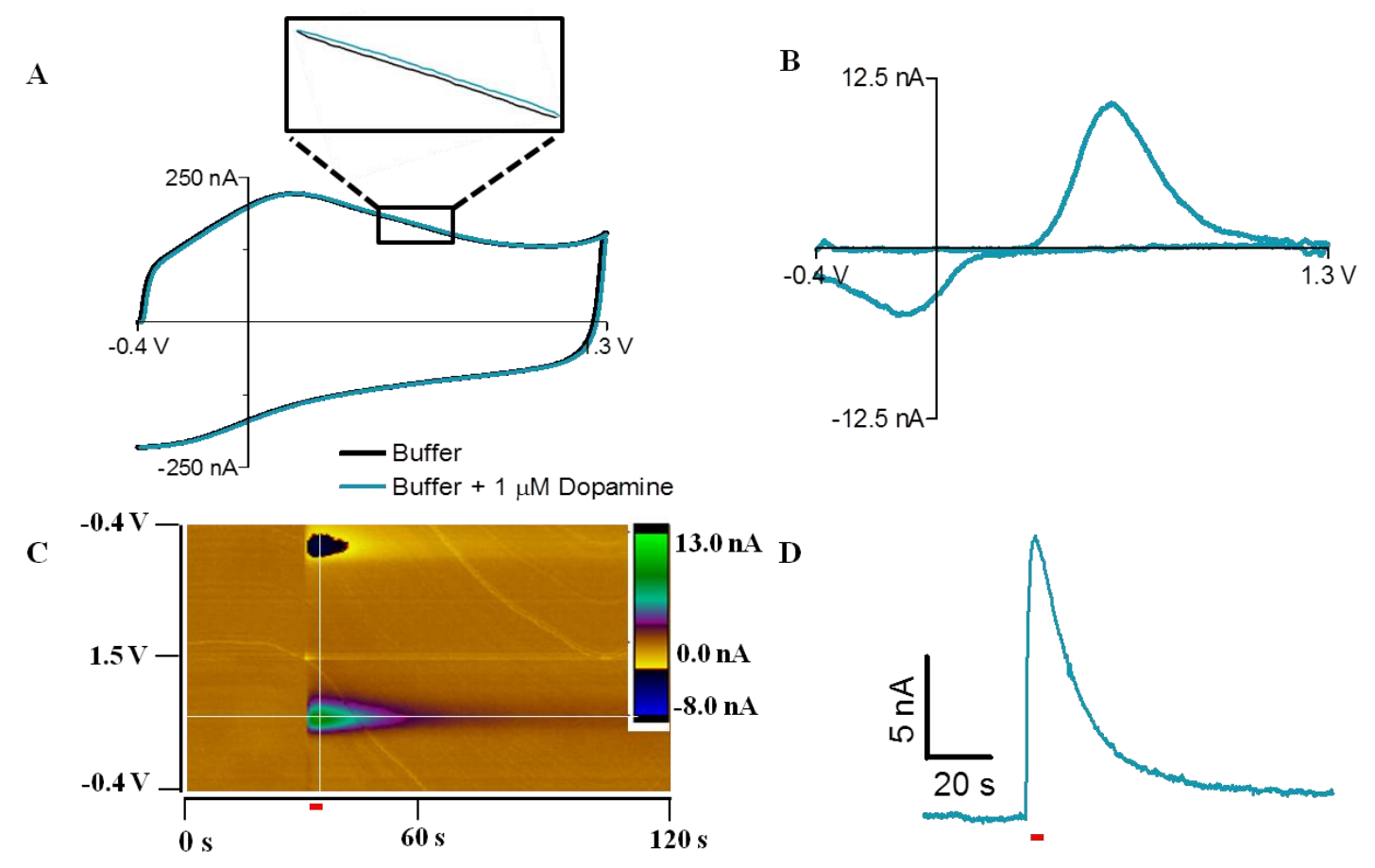

Figure 1.2: FSCV detection of dopamine. All data from exogenous dopamine in a Drosophila central nervous system. A) The black line is background current plotted against applied potential. The blue line is the current observed in the presence of dopamine. Inset shows a enlarged view of the dopamine oxidation current. B) The background current is subtracted from the current in the presence of dopamine resulting in a cyclic voltammogram for dopamine. The oxidation peak occurs near $0.6 \mathrm{~V}$ and the reduction peak occurs near $-0.2 \mathrm{~V}$. C) Color plot for dopamine detection. The $\mathrm{x}$-axis is time, the $\mathrm{y}$-axis is applied potential and current is shown in color. Current produced from oxidation reactions is shown in green, while current produced from reduction reactions is shown in blue. The red line shows when dopamine was applied to the central nervous system. The vertical white line shows the place where the cyclic voltammogram is taken from and the horizontal white line shows the place where the current vs time plot is taken from. D) Current vs. time plot showing the oxidation peak for dopamine. The signal decays as dopamine is removed by cellular processes.

In addition to the cyclic voltammogram and color plot, data can be visualized with a current vs. time plot. In Figure 1.2C, the horizontal white line runs through the potential at which maximal dopamine oxidation occurs. The resulting current vs. time plot is shown in Figure 1.2D. The red line shows the time course of dopamine release. The decay of current from maximal dopamine oxidation can be used to estimate the uptake or clearance of released dopamine by the dopamine transporter into dopaminergic neurons $(25,26)$. FSCV allows for chemical identity, and provides temporally resolved measurement of dopamine and information regarding dopamine uptake. 


\subsubsection{Carbon-fiber microelectrodes}

Carbon fiber microelectrodes (CFMEs) have been used extensively as biochemical sensors since the late 1970's $(27,28)$. In comparison to other biosensors, CFMEs have small dimensions, cost-effective construction, reduced biofouling, high surface area to volume ratios, and fast electron transfer rates. The carbon fibers used to make CMFEs are manufactured by spinning and stretching acrylic fibers, such as polyacrylonitrile, followed by heating the fibers to high temperatures $\left(>1000^{\circ} \mathrm{C}\right)$. During the heating process, the material becomes carbonized and heteroatoms are removed, yielding a material that is $>90 \%$ carbon (29). The resulting fibers have high tensile strength, are good conductors, and are biologically inert. In addition, oxygen-containing defect sites form along the length of the fibers, which provides electron transfer sites during electrochemical detection $(21,30)$.

CMFEs are constructed by aspirating a single carbon fiber into a glass capillary, and heating and pulling the capillary into two tapered electrodes. The carbon fiber protrudes out of the capillary at the tapered end and is trimmed either at the glass-fiber interface to form a disk-shaped electrode or at a short distance from the interface (40 $100 \mu \mathrm{m}$ ) to form a cylinder-shaped electrode (Figure 1.3). Both electrode geometries are commonly used with FSCV (18). The active area of disk electrodes is easy to calculate, making them more spatially resolved than cylinder electrodes; however, cylinder electrodes are more commonly used in vivo because they have a larger surface area and 
increased conduction along the length of the fiber, making them more sensitive than disk electrodes.

The average cylinder CMFE has tip diameter of 5-20 $\mu \mathrm{m}$, and an active length of 40-100 $\mu \mathrm{m}$, giving CMFE's high spatial resolution (Figure 1.3) (31). Other biosensors, such as microdialysis probes, are typically larger than 200 $\mu \mathrm{m}$, which make them less spatially resolved and more prone to cause tissue damage upon implantation (13).

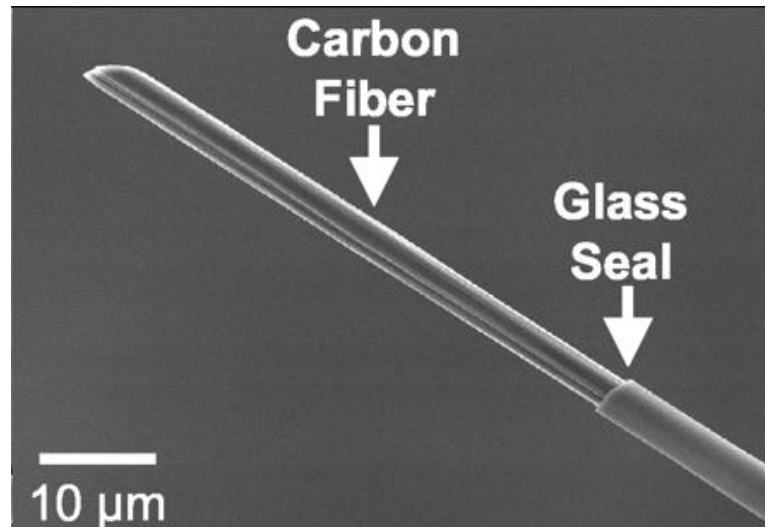

Figure 1.3: Scanning electron microscopic image of a cylindrical carbon-fiber microelectrode under $\times 1200$ magnification. The diameter of the fiber is $\sim 5 \mu \mathrm{m}$. The length of the exposed carbon fiber extending from the glass seal is the electroactive area of the electrode. Image from Robinson 2003.

Although electrodes can also be made from other materials, such as gold or platinum wire (on the order of tens of $\mu \mathrm{m}$ ), these electrodes are prone to biofouling, particularly in the presence of thiols. In addition, the potential window for redox reactions and dopamine detection with metal electrodes is smaller. For example, when scanning gold electrodes above potentials of $1.1 \mathrm{~V}$, corrosion currents are observed (32).

The CFME has negatively charged functional groups, such as hydroxyl and carbonyl, in aqueous environments. Therefore, at a physiological $\mathrm{pH}$ the $\mathrm{CMFE}$ is slightly more selective for positively charged compounds, such as dopamine. This selectivity, the small size of the fiber, and potential window make CFMEs good electrodes to use with FSCV for dopamine detection. 


\subsubsection{Comparison to other techniques}

In addition to FSCV, there are several common methods to measure dopamine such as imaging, microdialysis, amperometry, and chronoamperometry. Two major imaging methods are functional magnetic resonance imaging (fMRI) and positron emission tomography (PET). The benefit of imaging methods is that they are noninvasive and can be used with humans and non-human primates. In fMRI, brain activity in a particular region is measured by detecting the blood-oxygen-level-dependent signal. If an area of the brain is active, blood will flow to that area resulting in a change in oxygen levels (33). This technique has been used to study the role of dopamine in decision making and reward showing that dopaminergic activity in the striatum influences decision-making (34). However, the spatial resolution of this technique is limited to 2 to $3 \mathrm{~mm}$, and direct dopamine neurotransmission cannot be observed.

In PET neuroimaging, a radioactive ligand is injected into the blood stream. Once in the brain, the tracer emits positrons that are used to construct a three dimensional image of the brain and its activity. Several dopamine-specific radioligands, such as the dopamine antagonist $\left[{ }^{11} \mathrm{C}\right]$ raclopride, have been developed and used to study dopamine receptor activity. For example, PET has been used to show that the number of dopamine receptors decrease during chronic cocaine self-administration in non-human primates (35). However, PET imaging is not spatially resolved $(2.0 \mathrm{~mm})$. While the radiation from radiotracers is usually low, PET is commonly coupled with computed tomography (CT) in humans, which poses a risk of radiation exposure. Additionally, direct 
neurotransmission cannot be measured, nor can neurotransmitter concentrations be determined by this method.

Another common technique, microdialysis, has served as a powerful tool for the direct measurement of neurotransmitters. In microdialysis, a large probe (typically around $200-400 \mu \mathrm{m}$ ) with a semipermable membrane is implanted into the brain region of interest. The probe is perfused with artificial cerebral spinal fluid, and small molecules, such as neurotransmitters, diffuse across the membrane according to their concentration gradients. The fluid fractions are collected and typically separated using highperformance liquid chromatography or capillary electrophoresis. Coupling to separation techniques allows microdialysis to separate multiple analytes at one time and measure their analyte concentration simultaneously. Microdialysis has been used to determine that the basal level of dopamine is approximately $1-3.5 \mathrm{nM}(36,37)$. In addition, changes in basal levels of dopamine have been monitored using microdialysis to understand the effects of drugs of abuse, such as cocaine, on neurotransmission (38). Although microdialysis is sensitive and selective, the large size of the probe causes brain trauma and tissue damage, such as edema, adulteration of the blood-brain barrier, and glucose metabolism near the insertion site (39). Microdialysis also has low temporal resolution (usually on the order of $10 \mathrm{~min}$ ); however, recently, methods to improve temporal response have been developed $(40,41)$.

With constant-potential amperometry, a voltage sufficient to oxidize the analyte of interest is continuously applied to the electrode. As shown in Equation 1.3, current is proportional to concentration. Thus, currents can be measured continuously, which gives 
amperometry high temporal resolution. However, amperometry does not provide chemical selectivity. Any analyte, including electroactive interferents, that can undergo a redox reaction at the potential applied to the electrode, will be detected and will be indistinguishable from the analyte of interest. Therefore, amperometry is often used in measurements of single cells where the released substance is already known. For example, amperometry has been used extensively to detect dopamine during exocytosis at undifferentiated PC12 cells $(30,42-45)$.

Another voltammetric technique commonly used to detect dopamine is chronoamperometry. In chronoamperometry, a square waveform is applied to an electrode. An initial potential where no redox reactions occur is applied and then the voltage is stepped down to a potential sufficient to oxidize the analyte of interest. The current during the initial potential step is proportional to the concentration of analyte. As the potential is stepped back down, the analyte is reduced. The ratio of oxidative and reductive current is calculated for a particular time point. Because some analytes have unique ratios, this method has some chemical selectivity. Thus, chronoamperometry has been used to determine dopamine diffusion and uptake in the rat brain (46). However, the chemical resolution is not as good as that found with FSCV (7). In comparison to other techniques, FSCV offers a good mix of spatial and temporal resolution as well as chemical identity.

FSCV has a comparable temporal resolution to chronoamperometry, greater temporal resolution than microdialysis, but less temporal resolution than amperometry. In a complex, in vivo environment there are many compounds that are electroactive, and 
have the potential to interfere with dopamine detection; therefore, chemical identity is important (47). While amperometry is faster than FSCV, amperometry offers no chemical identity, and is not preferable when testing a new model system, such as Drosophila. Although FSCV offers greater chemical identity than amperometry, and greater speed than microdialysis, microdialysis offers the better chemical resolution. Several catecholamine neurotransmitters such as dopamine, norepinephrine, and epinephrine have similar cyclic voltammograms. These catecholamines cannot easily be distinguished from each other using FSCV, but can be using microdialysis. However, the timescale for microdialysis is too long to measure quick changes in neurotransmitter concentration, and the probes are too large for small tissue preparations, such as those used for Drosophila. In order to improve the chemical selectivity of FSCV and distinguish between similar compounds, pharmacological agents can be introduced that cause the analyte concentration to increase or decrease. For example, $\alpha$-methyl-DL-p-tyrosine (AMPT), an inhibitor of tyrosine hydroxylase, is often administered to confirm the identity of dopamine (1). If after drug administration, the concentration of evoked dopamine decreases and the cyclic voltammogram has redox peaks corresponding to dopamine, chemical identity is confirmed. Thus, FSCV is a suitable method for subsecond measurements in complex environments.

\section{2: Measuring Dopamine in vivo}

\subsection{1: Dopamine neurotransmission}


Dopamine is a neurotransmitter of interest because it is involved in human

behaviors such as movement, reward, addiction, appetite, arousal, learning and memory. In addition, atypical dopaminergic neurotransmission is involved in diseases such as Parkinson's disease, ADHD, schizophrenia, addiction, and restless leg syndrome. The exact mechanisms of dopaminergic signaling dysfunction for these diseases are not well understood. Therefore, studying the regulation of dopamine can lead to a better understanding of both human behavior and disease.

Neurons are composed of three main parts: the cell body, axons and dendrites (Figure 1.4). The cell body is where the nucleus of the cell is located, and the axons form connections called synapses with the cell bodies and the dendrites of other neurons. Neurons communicate with one another through either electrical or chemical transmission across a junction called a synapse. During electrical transmission, dendrites from adjacent neurons communicate through an electrical synapse (Figure 1.4). During chemical transmission,

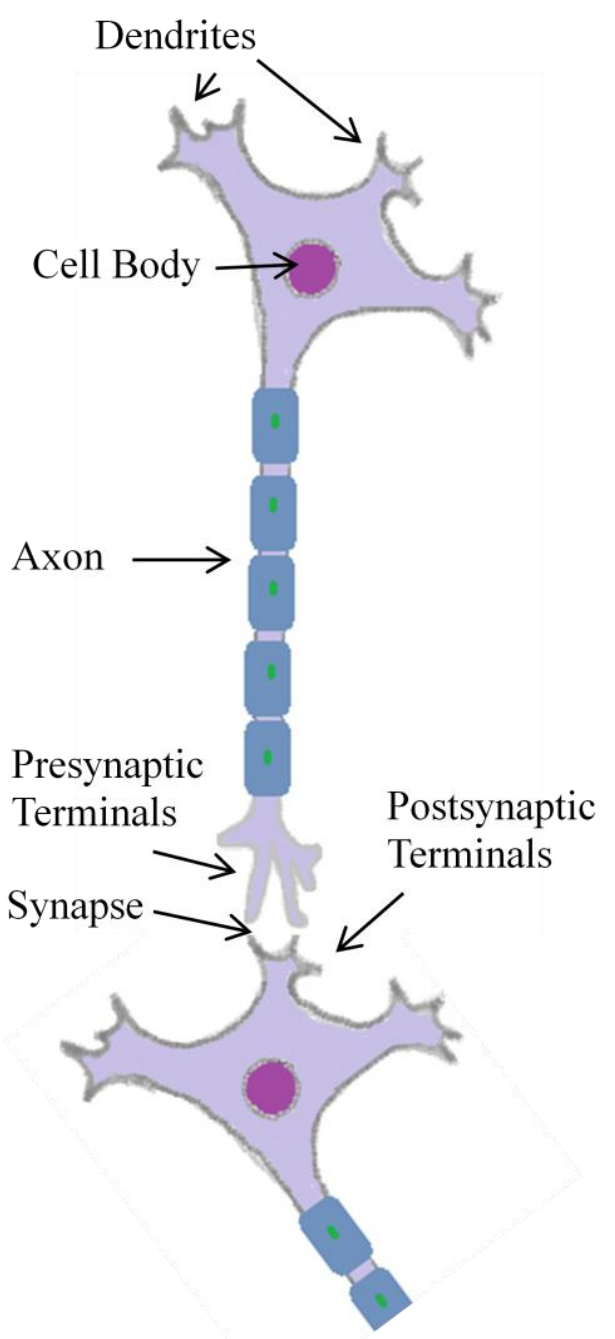

Figure 1.4: Major components of a neuron. Dendrites receive input from other neurons, the signal is propagated down the axon, and output to other neurons occurs at the terminals. 
neurotransmitters are released at the interface between an axon of one neuron and the dendrite of another neuron. The axon forms the presynaptic portion of the neuron and the dendrite forms the postsynaptic portion of the neuron. The region between the two neurons is called a synaptic cleft and is typically on the order of tens of nanometers. Neurotransmitter release is initiated by an action potential resulting from a change in potential across a cell membrane. Once initiated, the action potential propagates down the axon to terminals causing the opening of calcium ion channels. Calcium influx causes the release of neurotransmitter.

The catecholamine neurotransmitter, dopamine, is synthesized from the amino acid L-tyrosine (Figure 1.5). Once synthesized, dopamine is packaged into membranebound spheres called vesicles by the vesicular monoamine transporter protein. Upon exocytosis, the synaptic vesicles dock near the presynaptic membrane and dopamine is released into the synaptic cleft where it can interact with postsynaptic dopamine receptors, which perpetuate dopaminergic signaling. Dopamine can also interact with presynaptic dopamine receptors, which serve as autoreceptors regulating dopamine synthesis and exocytosis. Dopamine diffuses out of the synaptic cleft into the extracellular space and can be taken back into the dopaminergic neuron by the transmembrane protein called the dopamine transporter. Once taken back into the neuron, dopamine can be repackaged in vesicles or metabolized. 


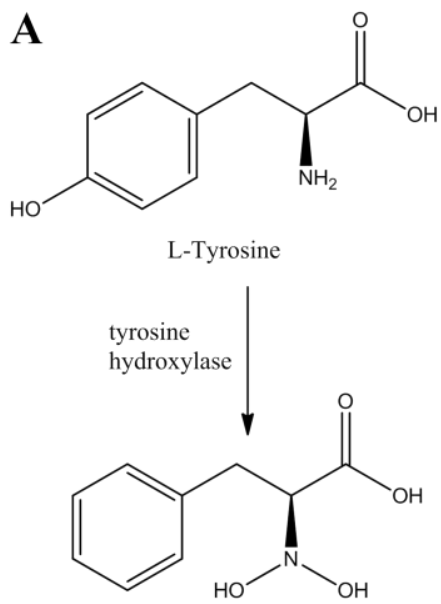

L-dihydroxyphenylalanine
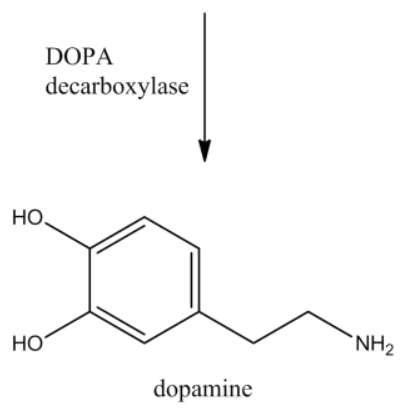

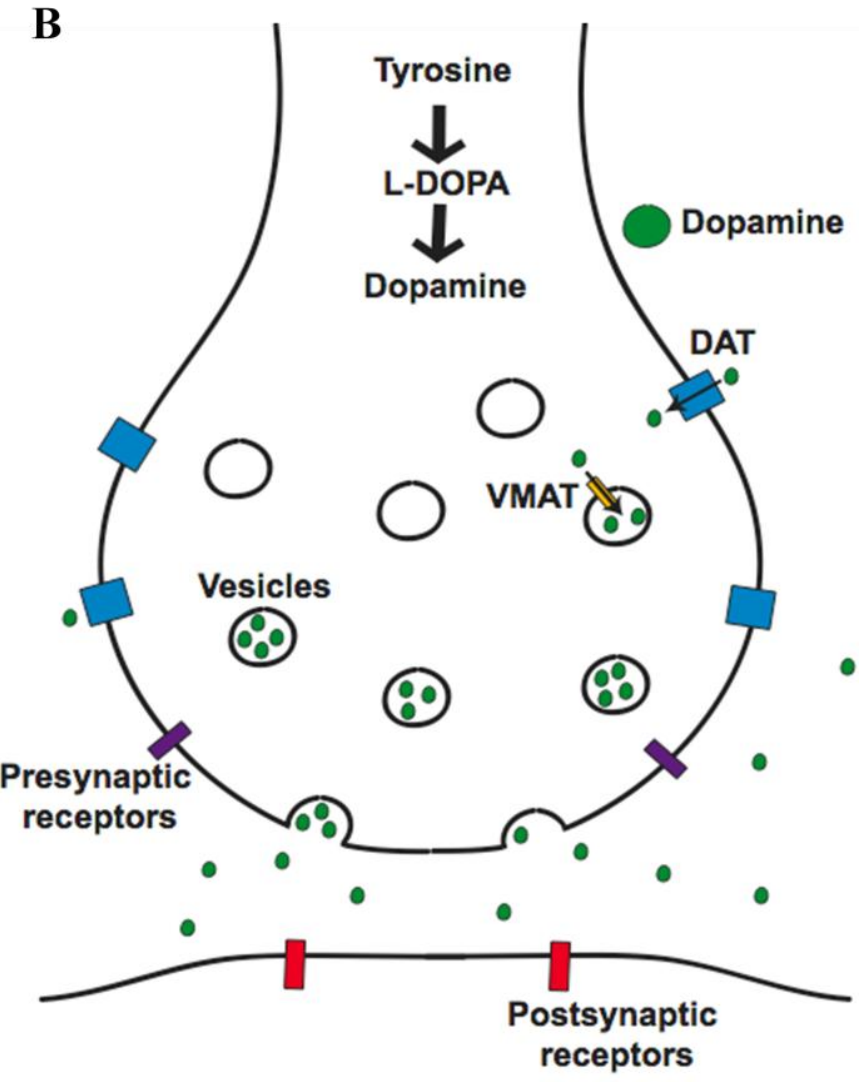

Figure 1.5: Dopaminergic Synthesis and Neuron. A) Dopamine synthesis pathway. B) Parts of the dopaminergic neuron involved in dopaminergic signalling.

Dopaminergic signaling between neurons occurs in two modes: tonic and phasic signaling $(14,48)$. During tonic signaling, a steady-state level of dopamine is achieved by slow dopamine release. Phasic signaling occurs on top of tonic signaling, and is caused by concerted burst firing of dopaminergic neurons, which leads to high concentrations of dopamine to be released over a brief periods of time, $<10 \mathrm{~s}(49)$. Because tonic signaling is slow and steady, microdialysis is commonly used to measure basal dopamine levels, which are estimated to be $1-3.5 \mathrm{nM}(36,37)$. Microdialysis is too slow to measure phasic signaling, which often occurs during behavior or sensory input. FSCV, on the other hand, 
is commonly used to measure phasic signaling, which can be induced by electrical stimulation in an anesthesititzed animal or introduction of novel stimuli in an awake, behaving animal (50). Understanding the dynamics of tonic and phasic dopaminergic signaling in animal models will help us understand, and eventually treat, the many diseases involving dopamine dysregulation.

\subsection{2: FSCV and dopamine detection}

As previously discussed, FSCV is an advantageous method to measure dopamine neurotransmission in vivo and in vitro. Wightman et al. conducted the first in vivo measurements of dopamine using FSCV in anesthetized rats (1). They electrically stimulated the median forebrain bundle of an anesthetized rat, giving rise to dopamine release in the striatum. Dopamine release was confirmed with a cyclic voltammogram as well as with pharmacology. Since this initial study, FSCV with electrically stimulated dopamine release has been used extensively because it mimics neuronal firing and is highly reproducible. Typically, electrically stimulated release occurs by stimulation of the substantia nigra, ventral tegmental area or the medial forebrain bundle using a stimulation train, and release is measured at an electrode implanted along the nerve terminals. FSCV with electrical stimulation has been used, mostly in rodent models, to characterize dopamine release, dopamine uptake kinetics, the role of dopamine receptors, and changes in dopamine concentration in the presence of pharmacological agents $(2,25,50)$. Studies using FSCV to detect dopamine, have led to an understanding of the underlying regional specificity of dopaminergic signaling. For example, FSCV has been used to determine 
how uptake of dopamine varies within brain regions. Analysis of clearance curves (the signal decay in Figure 1.2 D), has provided the maximum rate of uptake and transporter affinity for dopamine in various brain regions as well as in the presence of cocaine $(51$, 52).

FSCV has also been used to investigate dopamine neurotransmission in rat and mouse brain slices $(1,53,54)$. In these studies, thin slices of the brain are perfused with oxygenated artificial cerebral spinal fluid, an electrode is implanted into an area of interest, and release is electrically stimulated. Brain slices are often used to study the local effect of drug application. The exact concentration of the drug is known and systemic effects of the drug are eliminated. An advantage of using brain slices is that anatomical structures can be visualized more easily compared to using stereotaxic coordinates in whole animal preparations, allowing more precise electrode implantation. Brain slice experiments have been used to study dopamine uptake kinetics, and to study dopamine release in genetic mutants, such as dopamine transporter knock-out mice $(52$, $55)$.

Early FSCV studies focused on experiments in anesthetized animals and brain slice preparations. However, more recently, FSCV has been used to investigate dopamine transients or burst firing in awake, behaving rats. The first measurements of dopamine transients were reported by Rebec et al. (56). Upon entry to a novel environment, sharp increases in current at the oxidation potential of dopamine were observed. Subsequent studies, have measured dopamine levels during sexual arousal (49), novel stimuli (57), and cocaine self-administration (58). During self-administration of cocaine by rats, 
dopamine levels increased about $4 \mathrm{~s}$ before the lever press, and after the lever press (58). This observation shows that one role of dopamine is as an anticipatory signal. Real-time studies of dopamine transients in awake, behaving animals provide an idea of the role of dopamine in learning, reward and addiction.

Despite the advances made in understanding dopaminergic signaling using FSCV, the role that dopamine plays in human diseases is not fully understood, nor is the way that dopaminergic machinery such autoreceptors and transporters work together to regulate signaling. Although mouse lines mimicking human diseases, such as Parkinson's disease, are increasing in number, it still takes 1-2 years before these lines are available. Therefore, it would be advantageous to expand this method to other model organisms, such as Drosophila. This goal of this thesis is to open up the fly as a model system for studying basic mechanisms of dopamine neurotransmission using FSCV.

\subsection{3: Kinetic Modeling}

After dopamine is released, dopamine is removed from the extracellular space by the dopamine transporter. Recent studies have shown that uptake is the primary pathway for the clearance of released dopamine, and occurs on a second time scale (26). Uptake by the dopamine transporter can be modeled using the Michaelis-Menten equation (Equation 1.6) (59). At high concentrations, dopamine is transported back into the neuron at rate that is linear with time and that is given by the maximal rate of uptake, $V_{\max }$. At lower concentrations, the dopamine transporter is unsaturated and can be modeling using first order kinetics so that $v=\frac{V_{\max }}{K_{m}}(60)$. The substrate concentration at half of the 
maximal rate of uptake, $\mathrm{K}_{\mathrm{m}}$, can be used as an approximation for transporter affinity of DAT for dopamine. Low values of $K_{m}$ indicate high affinity and high values of $K_{m}$ indicate low affinity. The overall reaction rate can be described by:

$$
\begin{array}{rlr}
v=-\frac{d[D A]}{d t} & =\frac{V_{\max }[D A]_{\max }}{K_{m}+[D A]_{\max }} & \text { Equation 1.6 } \\
V_{\max } & =\text { maximal rate of dopamine uptake } & \\
K_{m} & =\text { substrate concentration at which the reaction rate is half } V_{\max } \\
{[D A]_{\max }} & =\text { maximal dopamine concentration }
\end{array}
$$

Dopamine release and uptake has been studied extensively in animal models using Michaelis-Menten kinetics. $V_{\max }$ and $K_{m}$ values have been determined for various regions of the rat brain, such as the caudate putamen, and release events from single nerve terminals have been studied. In addition, dopamine release and uptake in the presence of pharmacological agents has been studied. For example, psychostimulants such as cocaine and amphetamine inhibit dopamine transporter activity, prolong dopamine clearance and increase stereotypic behavior (52). Mice lacking functional dopamine transporters exhibit prolonged dopamine signaling and show limited responsiveness to dopamine transporter inhibitors; mice overexpressing dopamine transporters show increased rates of uptake and increased locomotor response to amphetamine $(5,61)$. While mice with genetically altered transporter expression levels can provide a model system to study dopamine release and uptake, mouse genetic models are difficult and time-consuming to make. Therefore, more tractable models are desirable.

\section{3: Drosophila}




\subsection{1: Drosophila as a model}

Simple model systems are useful in understanding basic neurobiological mechanisms. Models can help elucidate the genetic basis for a neurodegenerative disease, cellular mechanisms for disease phenotypes, and a model system for treatment. Since the early $20^{\text {th }}$ century, the fruit fly has been as a useful model system used to understand chromosome theory and developmental genetics. Studies looking at systematic disruption during development led to the discovery that genes essential to normal development were conserved between humans and Drosophila $(62,63)$. After both the human and Drosophila genomes were decoded, it was determined that $75 \%$ of all human disease genes have a Drosophila homolog, and approximately 700 out of 2,309 human disease genes are well-conserved enough to use Drosophila directly as a model (64). Many of these human diseases are neurological in origin and include Parkinson's, Alzheimer's and Huntington's disease.

Drosophila has been used to study neurotransmitter systems for over 30 years (65, 66). Many neurotransmitter systems are highly conserved between Drosophila and mammals. These include glutamate, GABA, dopamine, serotonin, acetylcholine, and histamine (67). The location and development of biogenic amines, such as dopamine, in Drosophila was first studied by Budnik et. al $(68,69)$, and refined by Lundell et. al $(70)$. The development of histochemical and immunocytochemical techniques allowed for the identification of aminergic neurons and biosynthetic pathways in the nervous system during all stages of Drosophila development (71-73). In addition to labeling, the ease of developing genetic models targeting a specific aminergic pathway or set of neurons has 
eased the barrier to understanding how neurotransmitter signaling precisely influences function and behavior. Currently, several thousand mutant flies are available from Drosophila stock centers. The biological conservation, short life span, and ease of making genetic models make Drosophila an excellent model.

\subsection{2: The UAS-GAL4 system}

One of the most useful genetic tools for Drosophila is the UAS-GAL4 system, which allows the over expression of a transgene or gene of interest in a specific tissue type (74). This system was developed by Andrea Brand and Norbert Perrimon in 1993 and consists of two parts: GAL4 and UAS (74). Gal4 is a protein from the yeast Saccharomyces cerevisiae. In yeast, GAL4 is responsible for regulation of galactose metabolism. In flies, GAL4 can activate transcription, but only under promoters that have GAL4 binding sites (75). The upstream activating sequence (UAS) is an enhancer that GAL4 specifically binds to activate transcription. Therefore, GAL4 is benign unless in the presence of UAS.

For this thesis, I bred a line of flies that express Channelrhodopsin-2 (ChR2), a blue-light activated cation channel found in blue-green algae, in neurons containing tyrosine hydroxylase (TH). A Drosophila line containing the TH-GAL4 mutation was crossed with a line containing the UAS-ChR2 (Figure 1.6). Once crossed, the GAL4 protein binds to UAS. Transcription of ChR2 is then activated in tissue containing tyrosine hydroxylase (Figure 1.6). 
Female
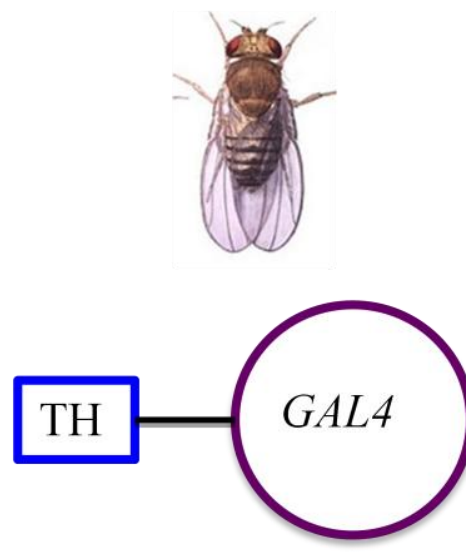

GAL4 Driver Line
Male
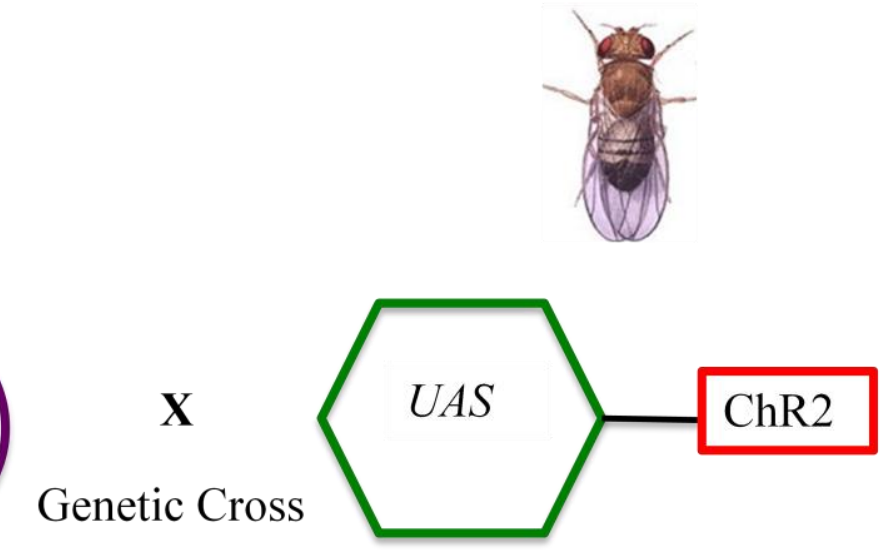

UAS Responder Line

\section{Gal4 protein}

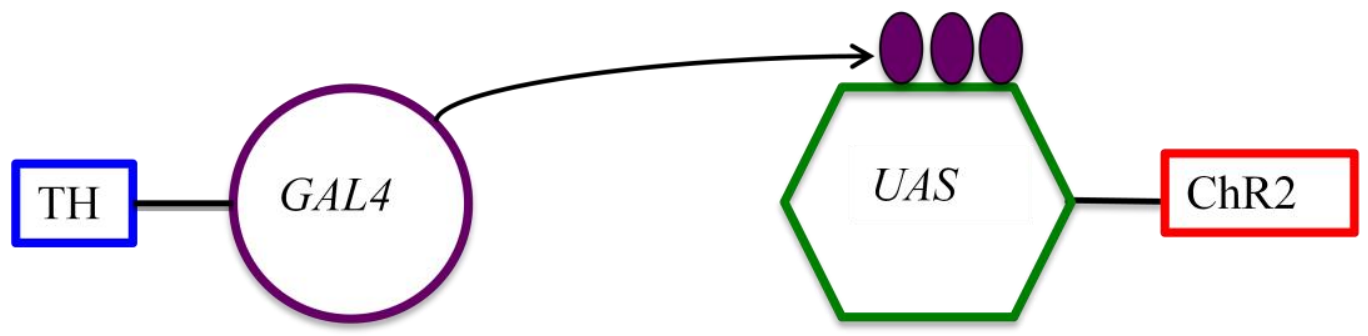

Figure 1.6: UAS-GAL4 system in Drosophila. Flies carrying the GAL4 enhancer gene are crossed with flies carrying the upstream activating sequence (UAS) gene. Once crossed, the GAL4 protein binds to UAS, which activates transcription of the transgene in the specific cell or tissue associated with GAL4.

\subsection{3: ChR2 and Drosophila}

Optical stimulation methods, which control cellular function with light, were pioneered by the Miesenbock lab $(76,77)$. Miesenbock used naturally occurring photosensitive proteins from Drosophila, and transfected them into both Xenopus oocytes and hippocampal cultures. Upon illumination, both cell types responded to the light, depolarizing for several seconds (76). The Miesenbock lab then expressed and successfully activated photosensitive proteins in Drosophila neurons responsible for 
escape movements such as jumping and wing beating (77). Around the same time, Nagel et al. showed that the seven transmembrane, light-gated ion channel from Chlamydomonas reinhardtii, ChR2, could also be used to depolarize cells with lightstimulation (78).

Due to the genetic palpability of Drosophila, Schroll et al. generated a transgenic Drosophila mutant expressing ChR2 under the control of the UAS-GAL4 system (79). The UAS-ChR2 lines allow expression of ChR2 in specific neuron types when UASChR2 lines are crossed with GAL4 driver lines. In the Schroll et al. study, ChR2 was expressed in motorneurons to show that distinct neuronal populations can be activated by blue light. ChR2 was then expressed in Th neurons and behavioral studies demonstrated that dopaminergic neurons paired with an odor stimulus induced aversive memory formation (79). The ability to use optical illumination to control neuron firing in Drosophila makes this organism a highly favorable model. Currently, there are thousands of GAL4 lines - many of these are dopamine-related, including those mimicking dopamine diseases. Therefore, developing a method to measure real-time dopamine release in disease models, will hopefully lead to a better understanding of the role that dopamine plays in these diseases.

\subsection{4: Dopamine in the larval ventral nerve cord}

Flies go through several stages of development (80). Embryogenesis and the first two larval stages (L1 and L2) are each 24 hours. The third larval stage takes two days followed by a pupae stage (4-5 days) and adulthood. Typically, larvae are hatched onto a 
food source. After reaching the third larval stage of development, larvae leave the food source and are called $3^{\text {rd }}$ instar wandering larvae (L3W). At this late stage of $\mathrm{L} 3$ development, the $3^{\text {rd }}$ instar larval central nervous system (CNS) becomes fully developed and persists into the adult stage (81). Thus, the $3^{\text {rd }}$ instar larval CNS can be used to study the neurobiology of a fully developed nervous system.

Figure 1.7A shows a light microscope image of a larval CNS that has been dissected from a wandering $3^{\text {rd }}$ instar larva. The larval CNS is composed of optic lobes (left) and a ventral nerve cord (VNC, right). The ventral nerve cord is bilaterally symmetric with individual segments that contain approximately 400 neurons. The three segments of the VNC are the subesophageal, thoracic and abdominal ganglia. Of the approximate 400 neurons, there are 80 neurons that have been identified as dopaminergic in the CNS (70). The dopaminergic cell pattern consists of four clusters of neurons in each side of the optic lobes, and a stereotyped pattered of lateral and medial neurons in each of the segments of the VNC.

Although the dopaminergic neurons are highly ordered throughout the CNS, their projections (dendrites and axons) extend throughout the CNS alongside other neuron types and their projections. Thus typical methods for inducing neurotransmitter release, which work by stimulating a specific neuron type, cannot be easily used. In addition, the size of the larval CNS is approximately $200 \mu \mathrm{m}$ by $50 \mu \mathrm{m}$ and $8 \mathrm{~nL}$. Both stimulating electrodes and microdialysis probes are on the order of $200 \mu \mathrm{m}$ and cannot be used with the Drosophila CNS. 
A

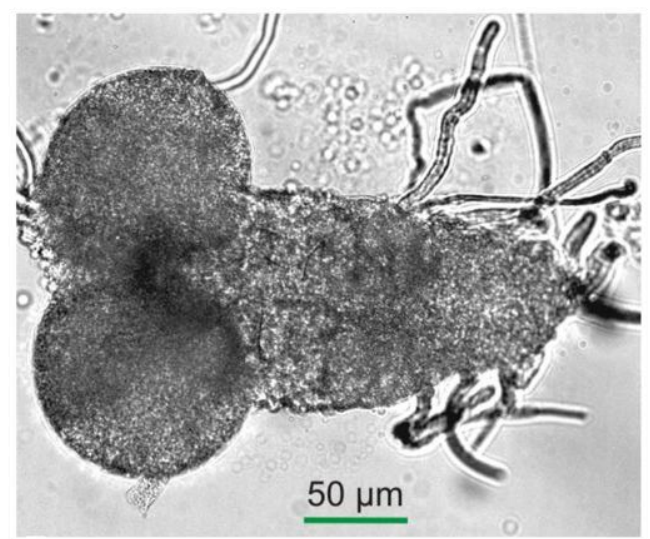

B

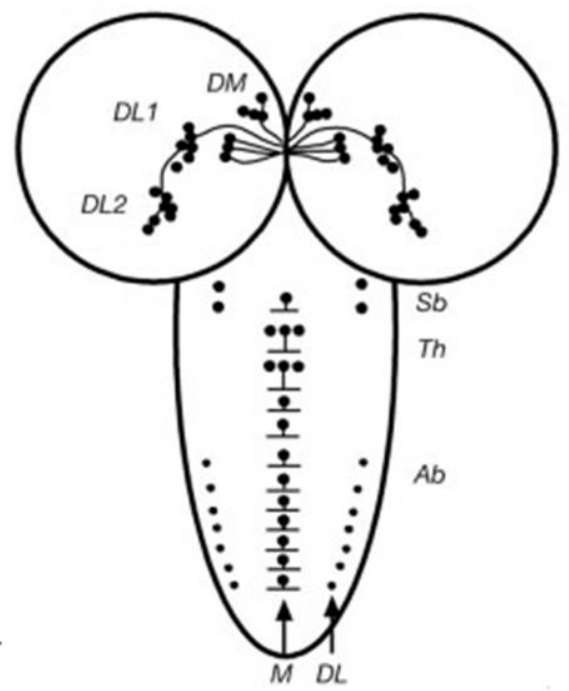

Figure 1.7: Drosophila anatomy. A) Light microscope picture of a 5-day-old, $3^{\text {rd }}$ instar larval CNS. The optic lobes are on the left and the nerve cord extends out. B) Schematic for dopaminergic neuron pattern in a $3^{\text {rd }}$ instar Drosophila larval CNS. Ab: abdominal ganglia, DL: dorsolateral neurons, DM: dorsomedial neurons, M: ventral medial neurons, Sb: subesophageal ganglion, Th: thoracic ganglia. Cell cluster nomenclature after Budnik 1988 for the larval CNS. Figure adapted from Hirsh 2003.

Analytical techniques for measuring dopamine in Drosophila have, therefore, focused on homogenization of one or more CNS samples followed by analysis with HPLC or capillary electrophoresis. Capillary electrophoresis with electrochemical detection can detect neurotransmitters, neuromodulators and metabolites from head homogenates, individual heads and individual brains (82-84). From such analyses, it has been reported that the larval CNS of wild type flies (Canton S) has dopamine concentrations ranging from $125 \pm 15$ to $74 \pm 2 \mathrm{fmol} / \mathrm{brain}(85)$. Analysis of tissue homogenates provides total neurotransmitter concentration, but this method lacks information regarding real-time signaling. 


\subsection{5: Using FSCV to characterize dopamine neurotransmission in Drosophila}

Direct, real-time measurement of dopamine signaling in Drosophila remains little understood despite the great potential for this model system to aid in understanding the underlying neurobiology of dopamine regulation. In this thesis, I develop a method to use FSCV at carbon-fiber microelectrodes for detection of real-time dopamine in the intact, larval CNS of Drosophila. This work overcomes a critical instrumentation barrier for measuring real-time dopamine release in Drosophila opening it up as a model system for studying mechanisms of neurotransmitter release. 


\section{4: Reference List}

1. Stamford, J. A., Kruk, Z. L., Millar, J., and Wightman, R. M. (1984) Striatal dopamine uptake in the rat: In vivo analysis by fast cyclic voltammetry, Neuroscience Letters 51, 133-138.

2. Bull, D. R., Bakhtiar, R., and Sheehan, M. J. (1991) Characterization of dopamine autoreceptors in the amygdala: A fast cyclic voltammetric study in vitro, Neurosci. Lett. 134, 41-44.

3. Pihel, K., Schroeder, T. J., and Wightman, R. M. (1994) Rapid and Selective Cyclic Voltammetric Measurements of Epinephrine and Norepinephrine as a Method To Measure Secretion from Single Bovine Adrenal Medullary Cells, Anal. Chem. 66, 4532-4537.

4. Jackson, B. P., and Mark Wightman, R. (1995) Dynamics of 5-hydroxytryptamine released from dopamine neurons in the caudate putamen of the rat, Brain Research 674, 163-166.

5. Giros, B., Jaber, M., Jones, S. R., Wightman, R. M., and Caron, M. G. (1996) Hyperlocomotion and indifference to cocaine and amphetamine in mice lacking the dopamine transporter, Nature 379, 606-612.

6. Garris, P. A., Budygin, E. A., Phillips, P. E. M., Venton, B. J., Robinson, D. L., Bergstrom, B. P., Rebec, G. V., and Wightman, R. M. (2003) A role for presynaptic mechanisms in the actions of nomifensine and haloperidol, Neuroscience 118, 819-829.

7. Robinson, D. L., Venton, B. J., Heien, M. L. A. V., and Wightman, R. M. (2003) Detecting Subsecond Dopamine Release with Fast-Scan Cyclic Voltammetry in Vivo, Clinical Chemistry 49, $1763-1773$.

8. Venton, B. J., and Wightman, R. M. (2007) Pharmacologically induced, subsecond dopamine transients in the caudate-putamen of the anesthetized rat, Synapse 61, 37-39.

9. Takmakov, P., Zachek, M. K., Keithley, R. B., Bucher, E. S., McCarty, G. S., and Wightman, R. M. (2010) Characterization of Local pH Changes in Brain Using Fast-Scan Cyclic Voltammetry with Carbon Microelectrodes, Anal. Chem. 82, 9892-9900. 
10. Maina, F. (2010) Functional fast scan cyclic voltammetry assay to characterize dopamine D2 and D3 autoreceptors in the mouse striatum, ACS Chem. Neuro. 1, $450-462$.

11. Herr, N. R., Park, J., McElligott, Z. A., Belle, A. M., Carelli, R. M., and Wightman, R. M. (2012) In vivo voltammetry monitoring of electrically evoked extracellular norepinephrine in subregions of the bed nucleus of the stria terminalis, Journal of Neurophysiology 107, $1731-1737$.

12. Armstrong-James, M., and Millar, J. (1979) Carbon fibre microelectrodes, Journal of Neuroscience Methods 1, 279-287.

13. Peters, J. L., Miner, L. H., Michael, A. C., and Sesack, S. R. (2004) Ultrastructure at carbon fiber microelectrode implantation sites after acute voltammetric measurements in the striatum of anesthetized rats, Journal of Neuroscience Methods 137, 9-23.

14. Wightman, R. M., and Robinson, D. L. (2002) Transient changes in mesolimbic dopamine and their association with "reward", Journal of Neurochemistry 82, 721-735.

15. Kawagoe, K., Zimmerman, J., and Wightman, R. (1993) Principles of voltammetry and microelectrode surface states., J Neurosci Methods 48, 225-40.

16. Venton, B. J., Michael, D. J., and Wightman, R. M. (2003) Correlation of local changes in extracellular oxygen and $\mathrm{pH}$ that accompany dopaminergic terminal activity in the rat caudate-putamen, Journal of Neurochemistry 84, 373-381.

17. Heien, M. L. A. V., Phillips, P. E. M., Stuber, G. D., Seipel, A. T., and Wightman, R. M. (2003) Overoxidation of carbon-fiber microelectrodes enhances dopamine adsorption and increases sensitivity, Analyst 128, 1413-1419.

18. Bath, B. D., Michael, D. J., Trafton, B. J., Joseph, J. D., Runnels, P. L., and Wightman, R. M. (2000) Subsecond Adsorption and Desorption of Dopamine at Carbon-Fiber Microelectrodes, Anal. Chem. 72, 5994-6002.

19. Kuhr, W. G., and Wightman, R. M. (1986) Real-time measurement of dopamine release in rat brain, Brain Research 381, 168-171.

20. Kile, B. M., Walsh, P. L., McElligott, Z. A., Bucher, E. S., Guillot, T. S., Salahpour, A., Caron, M. G., and Wightman, R. M. (2012) Optimizing the Temporal Resolution of Fast-Scan Cyclic Voltammetry, ACS Chem. Neurosci. 3, 285-292. 
21. Banks, C. E., Davies, T. J., Wildgoose, G. G., and Compton, R. G. (2005) Electrocatalysis at graphite and carbon nanotube modified electrodes: edge-plane sites and tube ends are the reactive sites, Chem. Commun. 829-841.

22. Bard, A. J., and Faulkner, L. R. (2001) Electrochemical methods : fundamentals and applications, Wiley, New York.

23. Bagotski, V. S. (2006) Fundamentals of electrochemistry, Wiley-Interscience, Hoboken, N.J.

24. Serway, R. A., and Jewett, J. W. (2004) Physics for scientists and engineers., Thomson-Brooks/Cole, Belmont, CA.

25. John, C. E., and Jones, S. R. (2007) Voltammetric characterization of the effect of monoamine uptake inhibitors and releasers on dopamine and serotonin uptake in mouse caudate-putamen and substantia nigra slices, Neuropharmacology 52, 1596-1605.

26. John, C. E., Budygin, E. A., Mateo, Y., and Jones, S. R. (2006) Neurochemical characterization of the release and uptake of dopamine in ventral tegmental area and serotonin in substantia nigra of the mouse, Journal of Neurochemistry 96, 267-282.

27. Ponchon, J. L., Cespuglio, R., Gonon, F., Jouvet, M., and Pujol, J. F. (1979) Normal pulse polarography with carbon fiber electrodes for in vitro and in vivo determination of catecholamines, Anal. Chem. 51, 1483-1486.

28. Gonon, F., Buda, M., Cespuglio, R., Jouvet, M., and Pujol, J.-F. (1980) In vivo electrochemical detection of catechols in the neostriatum of anaesthetized rats: dopamine or DOPAC?, Nature 286, 902-904.

29. Donnet, J.-B. (1998) Carbon fibers.

30. Chen, T. K., Luo, G., and Ewing, A. G. (1994) Amperometric Monitoring of Stimulated Catecholamine Release from Rat Pheochromocytoma (PC12) Cells at the Zeptomole Level, Anal. Chem. 66, 3031-3035.

31. Budai, D. (2010, January) Carbon Fiber-based Microelectrodes and Microbiosensors, Intelligent and Biosensors Chapter 14.

32. Zachek, M. K., Hermans, A., Wightman, R. M., and McCarty, G. S. (2008) Electrochemical dopamine detection: Comparing gold and carbon fiber microelectrodes using background subtracted fast scan cyclic voltammetry, Journal of Electroanalytical Chemistry 614, 113-120. 
33. Matthews, P. M., Honey, G. D., and Bullmore, E. T. (2006) Applications of fMRI in translational medicine and clinical practice, Nat Rev Neurosci 7, 732-744.

34. Nagano-Saito, A., Cisek, P., Perna, A. S., Shirdel, F. Z., Benkelfat, C., Leyton, M., and Dagher, A. (2012) From Anticipation to Action, the Role of Dopamine in Perceptual Decision-Making: an fMRI - Tyrosine Depletion Study, Journal of Neurophysiology.

35. Nader, M. A., Morgan, D., Gage, H. D., Nader, S. H., Calhoun, T. L., Buchheimer, N., Ehrenkaufer, R., and Mach, R. H. (2006) PET imaging of dopamine D2 receptors during chronic cocaine self-administration in monkeys, Nat Neurosci 9, 1050-1056.

36. Tang, A., Bungay, P. M., and Gonzales, R. A. (2003) Characterization of probe and tissue factors that influence interpretation of quantitative microdialysis experiments for dopamine, Journal of Neuroscience Methods 126, 1-11.

37. Parsons, L. H., Smith, A. D., and Justice Jr., J. B. (1991) The in vivo microdialysis recovery of dopamine is altered independently of basal level by 6hydroxydopamine lesions to the nucleus accumbens, Journal of Neuroscience Methods 40, 139-147.

38. Cadoni, C., and Chiara, G. D. (2007) Differences in dopamine responsiveness to drugs of abuse in the nucleus accumbens shell and core of Lewis and Fischer 344 rats, Journal of Neurochemistry 103, 487-499.

39. Clapp-Lilly, K. L., Roberts, R. C., Duffy, L. K., Irons, K. P., Hu, Y., and Drew, K. L. (1999) An ultrastructural analysis of tissue surrounding a microdialysis probe, Journal of Neuroscience Methods 90, 129-142.

40. Venton, B. J., Robinson, T. E., and Kennedy, R. T. (2006) Transient changes in nucleus accumbens amino acid concentrations correlate with individual responsivity to the predator fox odor 2,5-dihydro-2,4,5-trimethylthiazoline, Journal of Neurochemistry 96, 236-246.

41. Hotsenpiller, G., and Wolf, M. . (2003) Baclofen attenuates conditioned locomotion to cues associated with cocaine administration and stabilizes extracellular glutamate levels in rat nucleus accumbens, Neuroscience 118, 123134.

42. Cui, H.-F., Ye, J.-S., Chen, Y., Chong, S.-C., and Sheu, F.-S. (2006) Microelectrode Array Biochip: Tool for In Vitro Drug Screening Based on the Detection of a Drug Effect on Dopamine Release from PC12 Cells, Anal. Chem. 78, 6347-6355. 
43. Zhang, B., Heien, M. L. A. V., Santillo, M. F., Mellander, L., and Ewing, A. G. (2010) Temporal Resolution in Electrochemical Imaging on Single PC12 Cells Using Amperometry and Voltammetry at Microelectrode Arrays, Anal. Chem. 83, 571-577.

44. Kozminski, K. D., Gutman, D. A., Davila, V., Sulzer, D., and Ewing, A. G. (1998) Voltammetric and Pharmacological Characterization of Dopamine Release from Single Exocytotic Events at Rat Pheochromocytoma (PC12) Cells, Anal. Chem. 70, 3123-3130.

45. Finnegan, J. M., Pihel, K., Cahill, P. S., Huang, L., Zerby, S. E., Ewing, A. G., Kennedy, R. T., and Wightman, R. M. (1996) Vesicular Quantal Size Measured by Amperometry at Chromaffin, Mast, Pheochromocytoma, and Pancreatic $\beta$ Cells, J. Neurochem. 66, 1914-1923.

46. Zahniser, N. R., Dickinson, S. D., and Gerhardt, G. A. (1998) [48] High-speed chronoamperometric electrochemical measurements of dopamine clearance. In Neurotransmitter Transporters, pp 708-719, Academic Press.

47. Kissinger, P. T., Hart, J. B., and Adams, R. N. (1973) Voltammetry in brain tissue — a new neurophysiological measurement, Brain Research 55, 209-213.

48. Grace, A. A. (2000) The tonic/phasic model of dopamine system regulation and its implications for understanding alcohol and psychostimulant craving, Addiction 95, 119-128.

49. Robinson, D. L., Phillips, P. E. M., Budygin, E. A., Trafton, B. J., Garris, P. A., and Wightman, R. M. (2001) Sub-second changes in accumbal dopamine during sexual behavior in male rats, Neuroreport 12, 2549-2552.

50. Heien, M. L. A. V., Khan, A. S., Ariansen, J. L., Cheer, J. F., Phillips, P. E. M., Wassum, K. M., and Wightman, R. M. (2005) Real-time measurement of dopamine fluctuations after cocaine in the brain of behaving rats, P. Natl. Acad. Sci. USA 102, 10023-10028.

51. Garris, P. A., Ciolkowski, E. L., and Wightman, R. M. (1994) Heterogeneity of evoked dopamine overflow wihin the striatal and striatoamygdaloid regions, Neuroscience 59, 417-427.

52. Jones, S. R., Garris, P. A., and Wightman, R. M. (1995) Different effects of cocaine and nomifensine on dopamine uptake in the caudate-putamen and nucleus accumbens., J. Pharmacol. Exp. Ther. 274, $396-403$. 
53. Tso, M. M., Blatchford, K. L., Callado, L. F., McLaughlin, D. P., and Stamford, J. A. (2004) Stereoselective effects of ketamine on dopamine, serotonin and noradrenaline release and uptake in rat brain slices, Neurochemistry International $44,1-7$.

54. Stamford JA, Kruk ZL, and Millar J. (1988) Actions of dopamine antagonists on stimulated striatal and limbic dopamine release: an in vivo voltammetric study, Brit. J. Pharmacol. 94, 924-32.

55. Giros, B., Jaber, M., Jones, S. R., Wightman, R. M., and Caron, M. G. (1996) Hyperlocomotion and indifference to cocaine and amphetamine in mice lacking the dopamine transporter, Nature 379, 606-612.

56. Rebec, G. V., Christensen, J. R. ., Guerra, C., and Bardo, M. T. (1997) Regional and temporal differences in real-time dopamine efflux in the nucleus accumbens during free-choice novelty, Brain Research 776, 61-67.

57. Robinson, D. L., and Wightman, R. M. (2004) Nomifensine amplifies subsecond dopamine signals in the ventral striatum of freely-moving rats, Journal of Neurochemistry 90, 894-903.

58. Phillips, P. E. M., Stuber, G. D., Heien, M. L. A. V., Wightman, R. M., and Carelli, R. M. (2003) Subsecond dopamine release promotes cocaine seeking, Nature 422, 614-618.

59. Wightman, R. M., and Zimmerman, J. B. (1990) Control of dopamine extracellular concentration in rat striatum by impulse flow and uptake, Brain Research Reviews 15, 135-144.

60. Nicholson, C. (1995) Interaction between diffusion and Michaelis-Menten uptake of dopamine after iontophoresis in striatum, Biophysical Journal 68, 1699-1715.

61. Salahpour, A., Ramsey, A. J., Medvedev, I. O., Kile, B., Sotnikova, T. D., Holmstrand, E., Ghisi, V., Nicholls, P. J., Wong, L., Murphy, K., Sesack, S. R., Wightman, R. M., Gainetdinov, R. R., and Caron, M. G. (2008) Increased amphetamine-induced hyperactivity and reward in mice overexpressing the dopamine transporter, P. Natl. Acad. Sci. USA 105, $4405-4410$.

62. Morgan, T. H. (1915) The mechanism of Mendelian heredity, by T. H. Morgan [et al.], Holt,, New York,.

63. Lewis, E. B. (1978) A gene complex controlling segmentation in Drosophila, Nature 276, 565-570. 
64. Bier, E. (2005) Drosophila, the golden bug, emerges as a tool for human genetics, Nat Rev Genet 6, 9-23.

65. Uzzan, A., and Dudai, Y. (1982) Aminergic Receptors in Drosophila melanogaster: Responsiveness of Adenylate Cyclase to Putative Neurotransmitters, Journal of Neurochemistry 38, 1542-1550.

66. Dudai, Y. (1982) High-affinity octopamine receptors revealed in Drosophila by binding of $[3 \mathrm{H}]$ octopamine, Neuroscience Letters 28, 163-167.

67. Monastirioti, M. (1999) Biogenic amine systems in the fruit fly Drosophila melanogaster, Microsc. Res. Tech. 45, 106-121.

68. Budnik, V., and White, K. (1988) Catecholamine-containing neurons in Drosophila melanogaster: Distribution and development, J. Comp. Neurol. 268, 400-413.

69. Budnik, V., Martin-Morris, L., and White, K. (1986) Perturbed pattern of catecholamine-containing neurons in mutant Drosophila deficient in the enzyme dopa decarboxylase, The Journal of Neuroscience 6, 3682-3691.

70. Lundell, M. J., and Hirsh, J. (1994) Temporal and Spatial Development of Serotonin and Dopamine Neurons in the Drosophila CNS, Developmental Biology 165, 385-396.

71. Zalokar, M., and Erk, I. (1977) Phase-Partition Fixation and Staining of Drosophzla Eggs, Biotechnic \& Histochemistry 52, 89-95.

72. Mitchison, T. J., and Sedat, J. (1983) Localization of antigenic determinants in whole Drosophila embryos, Developmental Biology 99, 261-264.

73. Thomas, J. B., Bastiani, M. J., Bate, M., and Goodman, C. S. (1984) From grasshopper to Drosophila: a common plan for neuronal development, Nature 310, 203-207.

74. Brand, A. H., and Perrimon, N. (1993) Targeted gene expression as a means of altering cell fates and generating dominant phenotypes, Development 118, $401-$ 415.

75. Fischer, J. A., Giniger, E., Maniatis, T., and Ptashne, M. (1988) GAL4 activates transcription in Drosophila, Nature 332, 853-856.

76. Zemelman, B. V., Lee, G. A., Ng, M., and Miesenböck, G. (2002) Selective Photostimulation of Genetically ChARGed Neurons, Neuron 33, 15-22. 
77. Lima, S. Q., and Miesenböck, G. (2005) Remote Control of Behavior through Genetically Targeted Photostimulation of Neurons, Cell 121, 141-152.

78. Nagel, G., Szellas, T., Huhn, W., Kateriya, S., Adeishvili, N., Berthold, P., Ollig, D., Hegemann, P., and Bamberg, E. (2003) Channelrhodopsin-2, a directly lightgated cation-selective membrane channel, Proceedings of the National Academy of Sciences 100, 13940-13945.

79. Schroll, C., Riemensperger, T., Bucher, D., Ehmer, J., Völler, T., Erbguth, K., Gerber, B., Hendel, T., Nagel, G., Buchner, E., and Fiala, A. (2006) LightInduced Activation of Distinct Modulatory Neurons Triggers Appetitive or Aversive Learning in Drosophila Larvae, Current Biology 16, 1741-1747.

80. Hartenstein, V. (1993) Atlas of Drosophila development, Cold Spring Harbor Laboratory Press, Plainview, N.Y.

81. Truman, J. W., and Bate, M. (1988) Spatial and temporal patterns of neurogenesis in the central nervous system of Drosophila melanogaster, Developmental Biology 125, 145-157.

82. Paxon, T. L., Powell, P. R., Lee, H.-G., Han, K.-A., and Ewing, A. G. (2005) Microcolumn Separation of Amine Metabolites in the Fruit Fly, Anal. Chem. 77, 5349-5355.

83. Ream, P. J., Suljak, S. W., Ewing, A. G., and Han, K.-A. (2003) Micellar Electrokinetic Capillary Chromatography- Electrochemical Detection for Analysis of Biogenic Amines in Drosophila melanogaster, Anal. Chem. 75, 3972-3978.

84. Hardie, S. L., and Hirsh, J. (2006) An improved method for the separation and detection of biogenic amines in adult Drosophila brain extracts by high performance liquid chromatography, Journal of Neuroscience Methods 153, 243249.

85. Fang, H., Vickrey, T. L., and Venton, B. J. (2011) Analysis of Biogenic Amines in a Single Drosophila Larva Brain by Capillary Electrophoresis with Fast-Scan Cyclic Voltammetry Detection, Anal. Chem. 83, 2258-2264. 


\section{Chapter 2}

\section{Detection of endogenous dopamine changes in Drosophila melanogaster using fast-scan cyclic voltammetry}

I could not, at any age, be content to take my place by the fireside and simply look on. Life was meant to be lived. Curiousity must be kept alive. One must never, for whatever reason, turn his back on life. $\sim$ Eleanor Roosevelt 


\title{
Chapter 2: Detection of endogenous dopamine changes in Drosophila melanogaster using fast-scan cyclic voltammetry
}

\author{
Abstract \\ In this chapter, fast-scan cyclic voltammetry (FSCV) was used to develop a \\ method to detect endogenous dopamine in the fruit fly. Drosophila melanogaster, the \\ fruit fly, is a commonly used model organism because of its homology to mammals and \\ facile genetic manipulations. However, the size of the nervous system is very small. We \\ report a method to evoke and detect rapid changes in extracellular dopamine in a single \\ nerve cord isolated from a Drosophila larva. Flies were genetically modified to express \\ Channelrhodopsin-2, a blue-light activated cation channel, in only dopaminergic neurons. \\ Extracellular dopamine changes were measured with fast-scan cyclic voltammetry at an \\ implanted carbon-fiber microelectrode. Seven-second stimulations with blue light result \\ in an average peak dopamine concentration of $810 \pm 60 \mathrm{nM}$, similar to electrically- \\ stimulated release in mammals. Stimulations repeated at 15-minute intervals are stable \\ for 65 minutes, allowing pharmacological experiments in the same sample. Peak duration \\ is extended after cocaine or nisoxetine, inhibitors of the dopamine transporter (DAT). \\ Release was reduced upon exposure to reserpine, which inhibits vesicular packaging. \\ Chronic administration of NSD-1015, a dopamine synthesis inhibitor, decreased \\ dopamine release and inhibited pupation, showing a link between neurotransmission and \\ physiology. This is the first method to measure endogenous dopamine in an intact larval \\ Drosophila nervous system and will allow studies of genetic and pharmacological \\ manipulations of dopamine release and uptake.
}




\section{1: Introduction}

Drosophila melanogaster, the fruit fly, has been used for nearly 100 years as a model organism for understanding complex biological processes. For example, Drosophila has been used to study synaptic structure and neural circuitry (1-3) and as a model for human neurodegenerative diseases such as Amyotrophic lateral sclerosis, Parkinson's disease and Alzheimer's disease (4-6). About $75 \%$ of human genes have a functional ortholog in the fruit fly $(7,8)$. Drosophila has a short life span, large number of progeny, and can be easily genetically manipulated. These traits are amenable for the development of high throughput screenings because Drosophila genetic mutants can be created much quicker than in mammalian systems. For example, genetically-altered Drosophila can be produced in 1-2 months, while creating a knockout mouse can take up to 2 years. The limitation for using Drosophila to study neurotransmission has been lack of rapid measurement techniques.

Dopamine is an important neurotransmitter that is implicated in many human behaviors such as cognition, reward, addiction, motivation and motor function. Understanding dopamine regulation is essential for the treatment of many diseases including schizophrenia, attention-deficit disorder, and Parkinson's disease. Dopamine regulatory functions such as synthesis and uptake are conserved between humans and Drosophila $(9,10)$. Dopaminergic neurons have been characterized in Drosophila by immunohistochemistry $(11,12)$, which provides an understanding of neuronal morphology but not a direct measurement of neurotransmission. To quantitate dopamine tissue content in the fly central nervous system (CNS), tissue homogenates have been 
analyzed with HPLC or CE. These studies have estimated that total dopamine content ranges from 10 (13) to $74 \mathrm{pg}$ (14) in adult fly brains and $12-14 \mathrm{pg}(15)$ in the larval CNS.

While these methods can measure multiple neurotransmitters, homogenization destroys the tissue so repeated measurements cannot be made in the same sample. In addition, they do not measure the functional pool of dopamine that is released by exocytosis and acts in the extracellular space as a neurotransmitter.

Direct measurement of dopamine in an intact Drosophila melanogaster CNS has been hindered both by the small size of the CNS and lack of a method to evoke endogenous release specifically from dopaminergic neurons. For Drosophila, small probes are needed because the CNS is only $100 \mu \mathrm{m}$ wide and approximately $8 \mathrm{~nL}$ in volume. Therefore, the VNC is too small for both microdialysis probes and electrophysiology arrays, which are typically 100-200 $\mu \mathrm{m}$. Detection of fast changes in dopamine concentrations has traditionally been performed in mammalian systems using fast-scan cyclic voltammetry (FSCV) at carbon-fiber microelectrodes (16). FSCV is advantageous because of its high sensitivity and subsecond temporal resolution (17). The $7 \mu \mathrm{m}$ diameter carbon-fiber microelectrode is small enough to be implanted in the tiny fly CNS. The Ewing lab has developed a method using carbon-fiber microelectrodes to detect exogenously-applied dopamine in the adult Drosophila CNS (18). They evaluated dopamine clearance in normal flies and after pharmacological or genetic manipulation of the dopamine transporter (DAT), which is responsible for uptake. However, because this method does not measure endogenous dopamine, dopamine release cannot be studied. 
Recently, our lab has developed a microelectrode method to detect endogenous serotonin release and uptake in the intact ventral nerve cord of a single Drosophila larva (19). While mammalian experiments have often evoked release using electrical stimulation, the bipolar electrical stimulating electrode used in rodents is larger than the length of the entire larval Drosophila VNC. Utilizing Drosophila's genetic palpability, we have developed a method to evoke endogenous neurotransmitter release by genetically modifying flies to express Channelrhodopsin-2 (ChR2). ChR2 is a blue-light activated ion channel that can be inserted specifically into dopaminergic neurons using the commonly employed yeast GAL4/UAS system (20). Upon blue-light stimulation, ChR2 opens and the inward flow of calcium and sodium depolarizes the membrane, leading to exocytosis $(21)$.

In this study, we develop and characterize a method to measure dopamine release in a single, isolated larval Drosophila CNS. Dopamine release is evoked by blue-light activation of $\mathrm{ChR} 2$ and detected using FSCV at carbon-fiber microelectrodes. The advantages of this method are that endogenous dopamine can be measured repeatedly in the same sample in an intact Drosophila CNS. Characterization using pharmacological agents that disrupt synthesis, uptake and vesicular packaging shows that the Drosophila dopaminergic system is similar to mammals. In addition, chronic administration of a synthesis inhibitor also disrupts pupation, a physiological process requiring dopamine. Therefore, our technique can be used to measure physiologically relevant dopamine changes and will allow Drosophila to be utilized as a model system to study dopamine regulation. This chapter is adapted from a paper published in Analytical Chemistry (22). 


\section{2: Results and Discussion}

\subsection{1: Overview}

Dopaminergic signaling regulates a variety of complex behaviors in both flies and mammals including addiction, learning and sexuality $(23,24)$. In flies, dopamine also regulates pupation. Because these signaling pathways have been previously shown to be conserved between species, Drosophila is a good model system to study dopamine regulation $(10,25,26)$. We used 5 -day old, $3^{\text {rd }}$ instar larvae, which have been shown to have fully developed dopaminergic neurons $(12,27)$. Drosophila larvae are also advantageous over adult flies because their CNS is easily removed and immobilized.

\subsection{2: Anatomy of Drosophila dopamine neurons}

The larval Drosophila CNS is composed of optic lobes and a segmented ventral nerve cord (VNC). The CNS is small: approximately $100 \mu \mathrm{m}$ wide and $250 \mu \mathrm{m}$ in length. To visualize the location of dopamine neurons in a larva, a CNS was imaged from a fly expressing green-fluorescent protein in dopamine neurons. In Figure 2.1 A, the optic lobes are the large round features on the left and the ventral nerve cord (VNC) extends out from them. Dopaminergic cell bodies are visualized as white circles and are evident throughout the CNS. In the ventral nerve cord (box), which is similar to the spinal cord in vertebrates, many cell bodies are located near the midline (dashed line). On either side of the midline, there is a region called the neuropil composed of a dense network of 
synapses and neurotransmitter release sites including dopaminergic terminals. For electrochemistry experiments, the whole CNS was dissected from the larva and the optic lobes of the CNS were removed to facilitate electrode insertion. Dissection and incubation of CNS tissue in a modified Schneider's buffer has previously been shown to keep tissue viable for up to 6 hours after dissection (19, 27). The electrode was implanted in the neuropil to measure release from the dopamine terminals (Figure 2.1 B).

\subsection{3: Measurements of endogenous dopamine} evoked by Channelrhodopsin2 stimulation

In order to elicit release from only one type of neuron, we expressed Channelrhodopsin-2 (ChR2) specifically in dopaminergic neurons using the yeast-based $G A L 4 / \mathrm{UAS}$ system. Flies
A

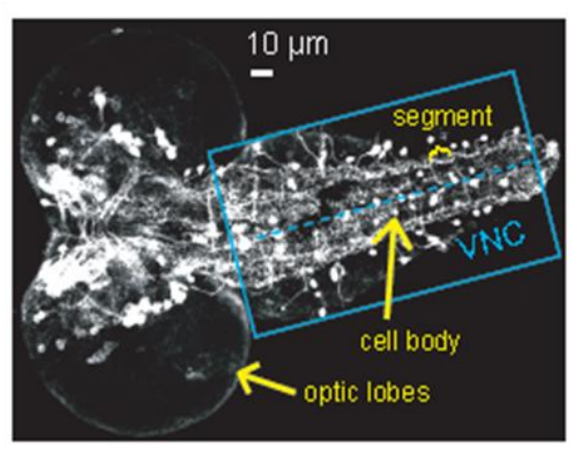

B

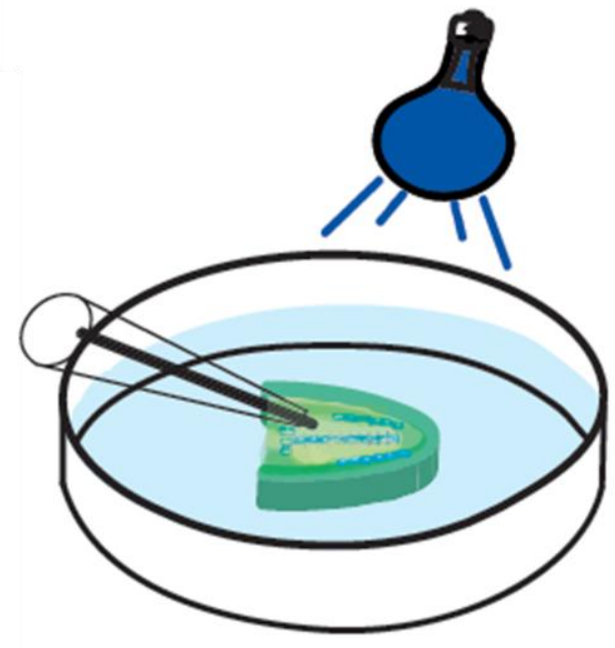

Figure 2.1: A) Fluorescence microscopy image of GFP-labeled dopaminergic neurons in a 5-day-old, $3^{\text {rd }}$ instar larva CNS. The blue box indicates the ventral nerve cord, and the dashed line marks the midline, where many cell bodies (white circles) are located. On either side of the midline is the neuropil region, which is rich in dopamine terminals. B) Schematic of microelecrode placement into the neuropil region of the ventral nerve cord with blue-light stimulation. The optic lobes have been removed from the CNS.

containing ChR2, which are controlled by the

upstream activating sequence (UAS) element, were crossed with flies containing GALA.

GAL4 was specifically targeted to cells containing tyrosine hydroxylase (th), a 
dopaminergic synthesis enzyme. Transcription of UAS requires GAL4; therefore, both UAS and GAL4 must be present in a cell for ChR2 to be expressed. The activation of ChR2 expressed in dopaminergic neurons causes depolarization and exocytosis (21).

This method of neuron-specific ChR2-mediated stimulation has numerous advantageous in comparison to electrical stimulation. First, it is specific and activates only one type of neuron. The dopaminergic projections are closely intertwined with other neurons, such as serotonergic projections, so electrical stimulation would be nonspecific. Second, larval dopaminergic neurons are small and the whole is nerve cord is smaller than most electrical stimulating electrodes, which are hundreds of microns in diameter. Finally, $\mathrm{ChR} 2$ stimulation is not action potential dependent, which might facilitate future studies that manipulate basal firing to determine the effects on stimulated release.

Evoked dopamine was measured at a carbon-fiber microelectrode that was inserted in the neuropil (Figure 2.1 B). A triangular waveform (-0.4 V to $1.3 \mathrm{~V}$ and back at $400 \mathrm{~V} / \mathrm{s}, 10 \mathrm{~Hz}$ ) was constantly applied to the electrode. Figure 2A shows cyclic voltammograms collected after a $7 \mathrm{~s}$ stimulation with blue light. The cyclic voltammogram collected from a larva expressing $\mathrm{ChR} 2$ exhibits a peak shape and redox features consistent with dopamine release. However, a cyclic voltammogram from a parent fly lacking the $\mathrm{ChR} 2$ insertion (th-GAL4) contains no characteristic oxidation or reduction peaks for dopamine. Therefore, $\mathrm{ChR} 2$ expression is required to elicit dopamine release. Figures 2.2 B and $\mathrm{C}$ show all the data collected in a three-dimensional falsecolor plot for the ChR2-expressing larva and the parent larva without ChR2, respectively. Consecutive cyclic voltammograms are plotted in time on the $\mathrm{x}$-axis and the applied 
voltage is plotted on the y-axis. Change in current is represented by color. In Fig. 2.2 B, the green area on the color plot corresponds to the oxidation of dopamine and the blue area to the reduction of dopamine. In the control sample with ChR2, a small change in current is observed at the switching potential $(1.3 \mathrm{~V})$ but there is an absence of dopamine oxidation or reduction peaks.
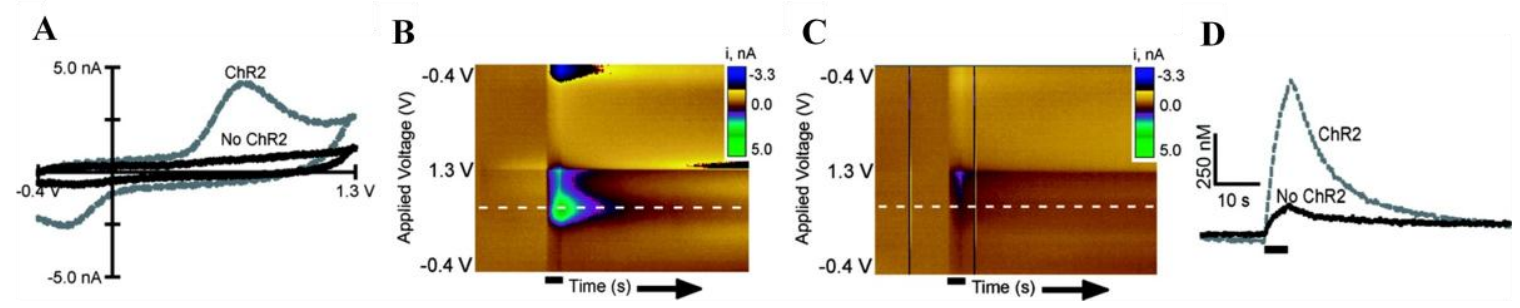

Figure 2.2: Characterization of dopamine signal evoked by ChR2 activation. Each larva was stimulated for $7 \mathrm{~s}$. The electrode was scanned from -0.4 to $1.3 \mathrm{~V}$ and back at $400 \mathrm{~V} / \mathrm{s}$ every $100 \mathrm{~ms}$. (A) Background subtracted cyclic voltammogram from a larva expressing ChR2 (dashed line) and one without ChR2 (solid line). The larva lacking the ChR2 insertion does not exhibit characteristic dopamine reduction and oxidization peaks. (B) Color plot showing a $7 \mathrm{~s}$ blue light stimulation (denoted by line under figure) in a larva expressing ChR2. The green and blue areas show the oxidation and reduction peaks, respectively, for dopamine. (C) Color plot from a larva without ChR2. The largest change in current corresponds to the switching potential. No dopamine peaks are present. The white dashed line marks the oxidation potential for dopamine. (D) Current vs. time plots show that the dopamine signal in a ChR2 expressing larva increases upon blue-light stimulation and decreases after the blue light is turned off. The duration of the blue light stimulus is marked as a line under the trace. The current at the oxidation potential has been converted to concentration using a postcalibration value. The larva without $\mathrm{ChR} 2$ has a small change at the oxidation potential for dopamine, not from dopamine release but due to ionic changes in response to blue light.

A concentration vs time profile can be plotted by converting the current at the maximal oxidation potential for dopamine to concentration using an in vitro calibration.

Figure 2.2 D shows the evoked dopamine signal in the ChR2-expressing VNC increases quickly upon stimulation and decays rapidly after the blue-light is turned off, which is consistent with evoked dopamine release and fast clearance. In the larva without the ChR2 insertion, a small change in current is observed when the current at the oxidation potential for dopamine is plotted over time (Figure 2.2 D). The change in current is not 
due to dopamine release, as confirmed by the cyclic voltammogram, but is detected at all potentials. This error is observed upon exposure to blue light, and not other wavelengths, and is likely due to changes in the background current caused by ionic changes during blue light activation. The current change in larva without ChR2 is only about $13 \%$ of the average stimulated release and defines the lower limit of detection $(\sim 110 \mathrm{nM})$.

The average concentration of evoked dopamine measured in ChR2 expressing larvae is $810 \pm 60 \mathrm{nM}(\mathrm{n}=7)$, which is similar to concentrations observed in mammalian experiments. For example, stimulating a rat brain slice with a single electrical pulse results in approximately $2300 \pm 400 \mathrm{nM}$ dopamine in the caudate-putamen and from 540 $\pm 90 \mathrm{nM}$ to $1500 \pm 600 \mathrm{nM}$ in the nucleus accumbens core $(28,29)$. In vivo, peak dopamine concentrations between 250 and $600 \mathrm{nM}$ have been observed after short, electrical stimulation pulse trains ( 0.4 to $2 \mathrm{~s}$ duration) (30-32). This similarity in magnitude of endogenous dopamine release between Drosophila and mammals validates using Drosophila as a model system. Longer stimulations were necessary to evoke ChR2mediated neurotransmitter changes in Drosophila similar to electrically-stimulated experiments in mammals. This is most likely due to the low wattage of the halogen lamp and the difficulty of the light to penetrate the outermost glial cell layer of the nerve cord. Additionally, the extent to which ChR2 is expressed is unknown, so not all neurons may be depolarized as with electrical stimulation.

\subsection{4: Effects of stimulation parameters on evoked dopamine release.}


Stimulated release must be stable over a prolonged time interval in order to evaluate changes induced by pharmacological agents in the same sample. Stability parameters were established by varying stimulation length and recovery time. A stable, reproducible signal was achieved with repeated $7 \mathrm{~s}$, blue-light stimulations every 15 minutes. For example, Figure 2.3 A shows an initial $7 \mathrm{~s}$ stimulation and the fifth stimulation evoked 60 minutes later in the same VNC. The peak concentration evoked by each stimulation is nearly identical. Figure 3B demonstrates that repeating 7 -s long stimulations at 15 -minute intervals produces stable release for approximately 65 minutes. At longer times, evoked dopamine begins to decrease.

In order to evaluate the stability of the time course of signaling, the time to half decay $\left(t_{50}\right)$ was calculated from the individual current versus time profiles. $t_{50}$ is defined as the time from the end of the stimulus until the half maximal concentration is reached (Figure $2.3 \mathrm{C}$ ). This value can be used as an estimate of uptake by the dopamine transporter. Figure $2.3 \mathrm{D}$ shows $\mathrm{t}_{50}$ is constant for approximately 65 minutes, demonstrating that uptake is stable. Prolonged stability of both release and uptake during stimulated release experiments confirms that Drosophila is a viable model system for making repeated measurements of dopamine in the same sample. 

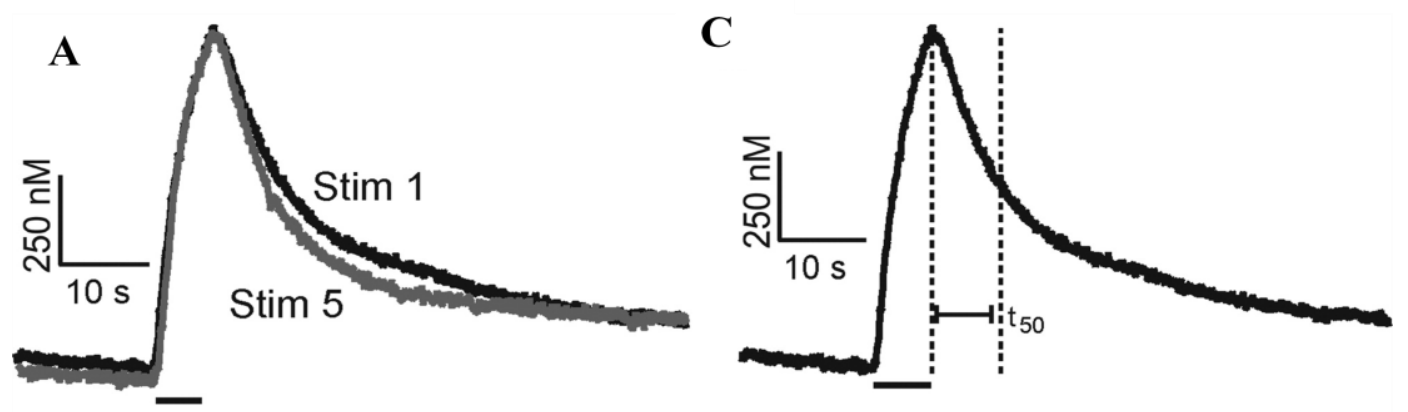

B

D
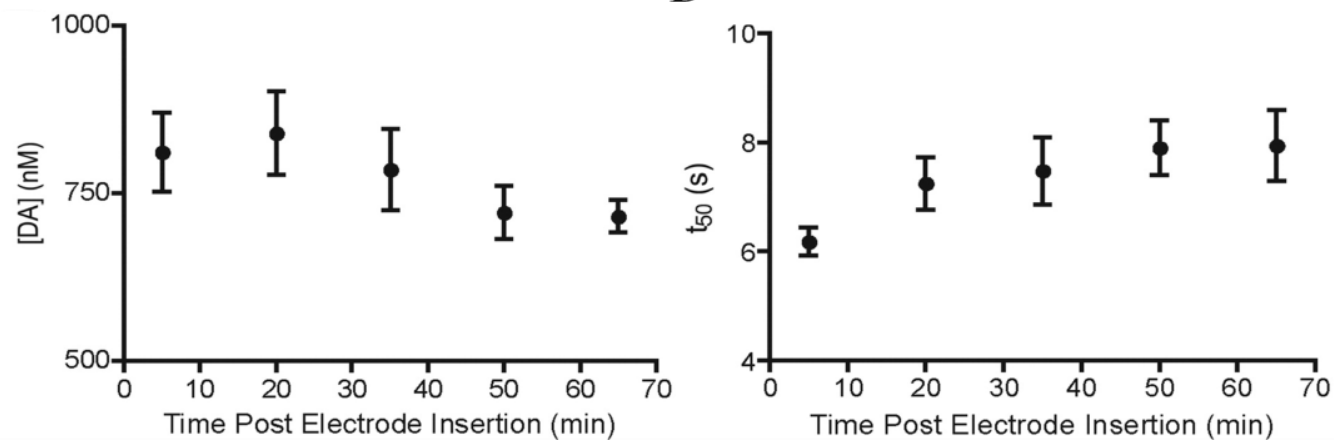

Figure 2.3: Effect of repeated stimulations. Each VNC was stimulated for $7 \mathrm{~s}$ at 15 min intervals. (A) Concentration vs time profiles from stimulations within the same VNC taken for $60 \mathrm{~min}$. (B) Pooled data (mean $+/-$ SEM, $n=7$ ) show that the evoked dopamine concentration is stable for over an hour following nerve cord isolation. (C) Diagram of the definition of $t 50$. The half decay time is defined as the time from the end of the stimulation until the signal decays to $50 \%$. (D) Pooled data (mean $+/-$ SEM $n=$ 7) show that $t 50$ is stable for 65 min.

To test the effect of stimulation length on evoked dopamine, the duration of the blue-light stimulation was varied. VNCs were exposed to $3,5,7,10,12$ or 15 s of bluelight stimulation, in that order, with 15 minutes rest between stimulations to allow the releasable pool of dopamine to recover (Figure 2.4). As the duration of activation increases, the amount of dopamine released also increases until a saturation point is reached around $10 \mathrm{~s}$. For stimulations over $10 \mathrm{~s}$, the peak height plateaus but the duration of signaling is longer. This suggests that either a maximal amount of release has been reached or that uptake is able to clear enough of the released dopamine during the longer stimulations that a steady-state curve is evident. Previously, ChR2 activation has been 
shown to occur on a millisecond time scale after which the channel reaches a steady state

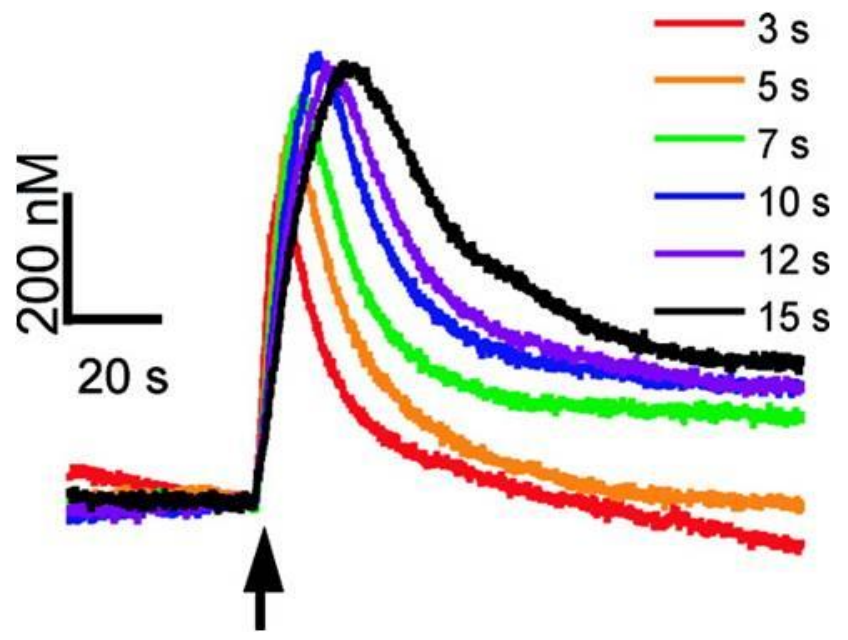

Figure 2.4: Effect of stimulation duration on dopamine signaling. Stimulations were performed for $3,5,7,10$, 12 , or $15 \mathrm{~s}$ in one ventral nerve cord with $15 \mathrm{~min}$ of recovery time in between each stimulation. The stimulation start time is indicated by the black arrow.
(33). Once open, ChR2 is responsive

to continuous light for almost a full

minute. Therefore, it is unlikely that

the plateaus in peak height observed

for longer stimulations are a result of desensitization of ChR2. A complete

return by the signal to baseline is not

observed for every stimulation,

which is commonly observed in in

vitro FSCV experiments (34).

Therefore, the $7 \mathrm{~s}$ stimulation was chosen for pharmacology experiments, due to its robust, reproducible signal. However, in future experiments, 5 s stimulations may also be useful because evoked dopamine is well below maximal peak height.

\subsection{5: Characterization of dopamine release using pharmacology}

While fast-scan cyclic voltammetry provides a CV that allows chemical identification of the measured species, many neurotransmitters have CVs that are difficult to distinguish. For example, dopamine and norepinephrine have identical CVs, although the fly does not contain norepinephrine or epinephrine (23) so they are not possible interferents in Drosophila. Another method to identify the species being detected is to pharmacologically manipulate the specific neurotransmitter system (35). Therefore, in 
order to verify that dopamine was being detected, we inhibited dopamine synthesis pharmacologically with NSD-1015, which blocks the aromatic L-amino acid decarboxylase (AADC) enzyme. AADC catalyzes the decarboxylation of L-DOPA into dopamine, which is the second step in the dopamine synthesis pathway.

Twenty $\mu$ M NSD-1015 was mixed with yeast and fed to larvae for 2 days prior to the experiment. When a larva is chronically exposed to NSD-1015, the amount of evoked dopamine decreases significantly (Figure 2.5 A). The concentration of dopamine measured on the initial stimulation was $410 \pm 80 \mathrm{nM}(\mathrm{n}=6)$, significantly less than normal flies (unpaired t-test, $p=0.0038$, Figure 5B). While stimulations repeated at 15 minute intervals evoke constant release in normal flies, subsequent stimulations from larvae fed NSD-1015 showed even lower release. For example, the second stimulation evokes on average only $43 \pm 8$ percent of the first, a significant decrease $(n=5$, paired $t$ test, $\mathrm{p}=0.004)$. The decrease in evoked release after NSD-1015 demonstrates that the measured neurotransmitter is dopamine and that synthesis is necessary to maintain the releasable pool of dopamine.

In order to pupate, Drosophila metabolizes dopamine into melanin (23). Interestingly, larvae fed NSD-1015 exhibited delayed pupation by 3-4 days or failed to pupate at all. Our electrochemistry data show a loss of releasable dopamine and the lack of pupation demonstrates that lower dopamine has a physiological effect. Because NSD1015 was chronically administered to larvae, this technique is suitable for future studies of the effects of chronic drug exposure on dopamine levels. 

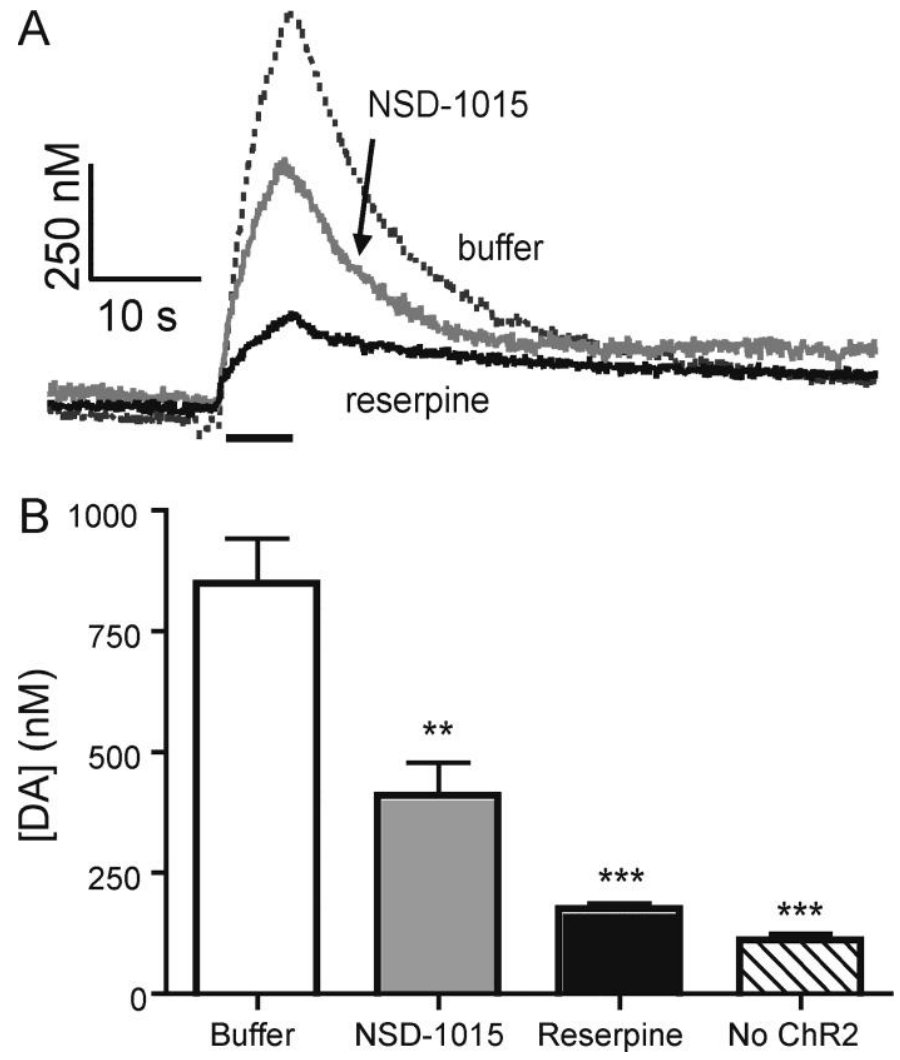

Figure 2.5: Effect of pharmacological agents on evoked dopamine concentration. (A) Concentration vs time profiles from individual VNCs show that peak dopamine concentration is reduced when larva are chronically fed NSD$1015(6.6 \mu \mathrm{M})$, a synthesis inhibitor, or are incubated in reserpine $(100 \mu \mathrm{M})$, a VMAT inhibitor that disrupts vesicular packaging. (B) The concentration of dopamine elicited by the first stimulation is significantly less for NSD$1015(* *, p<0.01)$ and reserpine $(* * *, p<0.001)$ than in samples dissected in buffer but more than control experiments where $\mathrm{ChR} 2$ was not expressed. $*, p<0.05 ; * *, p$ $<0.01 ; * * *, p<0.001$.

To test whether the dopamine release is vesicular, we dissected and incubated VNCs in reserpine. Reserpine inhibits the vesicular monoamine transporter (VMAT) and prevents dopamine from being packaged into vesicles. In mammals, reserpine has been shown to diminish stimulated dopamine release (36). Because Drosophila possess a functional ortholog of VMAT, the isoform dVMAT-A, the response to reserpine should be conserved between species (37). Figure 5A shows that 30 min incubation in reserpine significantly reduces dopamine signaling. Evoked release was $180 \pm 10 \mathrm{nM}$, significantly less than buffer-incubated animals ( $\mathrm{n}=7$, unpaired t-test, $\mathrm{p}<0.0001)$, but higher than in control subjects without $\operatorname{ChR} 2(\mathrm{n}=7$, unpaired t-test, $\mathrm{p}<0.0001)$, indicating that a small amount of dopamine is still detected (Figure 2.5 B). Because reserpine effectively 
reduced the evoked dopamine signal, dopamine release is indeed vesicular. Therefore, Drosophila is useful in studying mechanisms of vesicular packaging and exocytosis.

\subsection{6: Characterization of dopamine uptake using pharmacology}

Dopamine is taken up from the extracellular space back into the cell by the dopamine transporter (DAT). Therefore, if the transporter is blocked, the dopamine clearance rate decreases and dopamine signaling is prolonged, which has been observed in mammalian systems (38). To test the effect of blocking uptake in Drosophila, VNCs were dissected and incubated in $50 \mu \mathrm{M}$ cocaine, a drug of abuse, and known DAT inhibitor $(25,38)$. Figure 2.6A shows example traces of dopamine after uptake inhibition. The evoked peak height is similar for the cocaine and buffer traces, but the signal takes longer to return to baseline after cocaine. The peak concentration elicited by the initial stimulation was $910 \pm 80 \mathrm{nM}$, which is not significantly different from buffer controls $(n=7$, unpaired $t$ test, $p=0.6107)$ (Figure 2.6B). The half decay time $\left(t_{50}\right)$ for cocaine-exposed nerve cords increased by nearly $50 \%$ and was significantly larger than buffer-incubated controls ( $n=6$, unpaired t-test, $p=0.0001$, Figure 2.6C), which indicates uptake plays a role in dopamine clearance. 
While cocaine has been traditionally used to inhibit DAT in mammals, in

Drosophila, cocaine has a higher affinity for the serotonin transporter (SERT) than DAT

(21). Therefore, to prove that DAT is specifically responsible for dopamine clearance, we

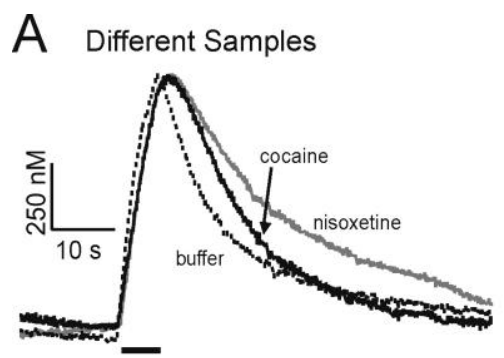

D same Sample
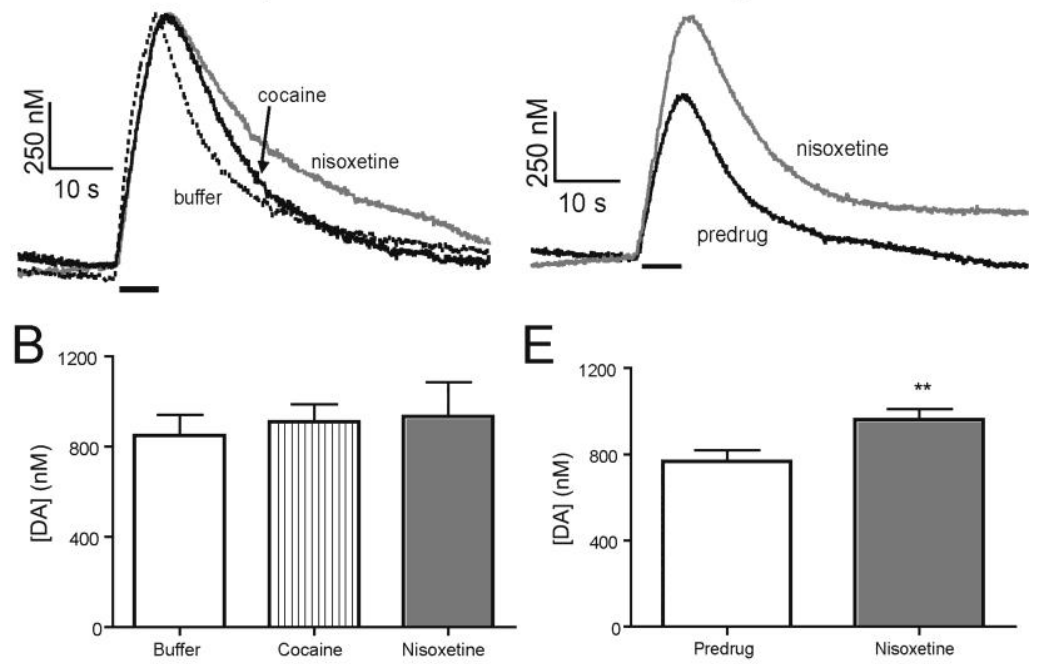

$\mathrm{E}$

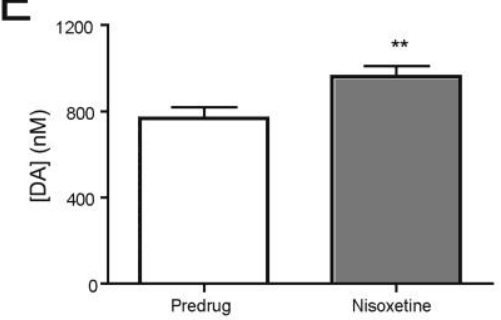

C

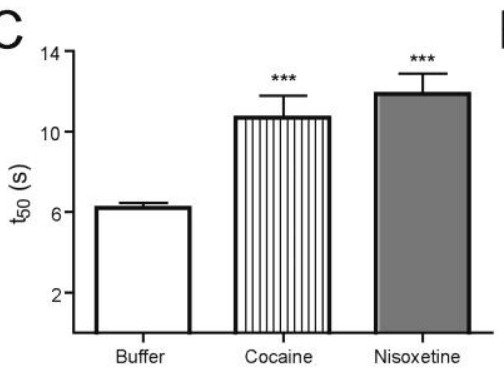

$\mathrm{F}$

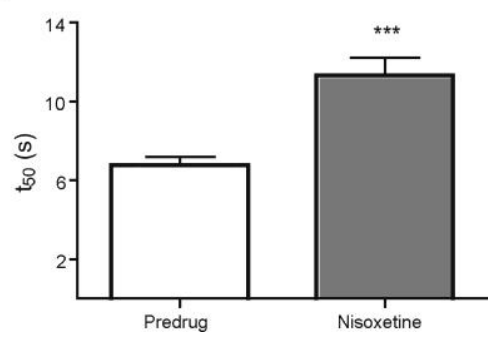

performed measurements

in a different experimental

group that was treated

with nisoxetine.

Nisoxetine has a $K_{i}$ value

three orders of magnitude

higher for DAT than

SERT in Drosophila (5.6

and $5000 \mu \mathrm{M}$,

respectively). An example

trace after $20 \mu \mathrm{M}$

nisoxetine shows an

Figure 2.6: Effect of DAT inhibitors on dopamine signaling. (A) Concentration versus time profiles from individual VNCs dissected in buffer or incubated in $50 \mu \mathrm{M}$ cocaine or $20 \mu \mathrm{M}$ nisoxetine. DAT inhibition increases the clearance time for dopamine. (B) The initial concentration of dopamine measured in VNCs in the presence of 50 $\mu \mathrm{M}$ cocaine $(n=6)$ or $20 \mu \mathrm{M}$ nisoxetine $(n=6)$ was not significantly different than that measured in buffer $(n=7)$. (C) The half decay time ( $t 50)$ for VNCs dissected in buffer $(n=7)$ is significantly less $(* * *, p<0.001)$ than for cocaine $(n=6)$ or nisoxetine $(n=6)$. (D) Concentration versus time profiles from a same-sample experiment. An initial $7 \mathrm{~s}$ blue-light stimulation was performed, then the nerve cord was incubated in $20 \mu \mathrm{M}$ nisoxetine, and another stimulation was performed $30 \mathrm{~min}$ later. DAT inhibition increases the clearance time and concentration of dopamine. (E) Evoked release significantly increased $(* *, p<0.01)$ in the same-sample experiment after nisoxetine was added $(n=6)$. (F) The half decay time $(t 50)$ significantly increased $(* * *, p<0.001)$ after nisoxetine. increase in clearance time

compared to samples

incubated in buffer (Figure

2.6 A). The concentration

of evoked dopamine after

nisoxetine incubation is 930 
$\pm 150 \mathrm{nM}$, which is not significantly different from the buffer group ( $\mathrm{n}=7$ unpaired $\mathrm{t}$ test, $\mathrm{p}=0.6351)$ (Figure 6B). Nisoxetine also significantly increased $\mathrm{t}_{50}$ (Figure 2.6C, $\mathrm{n}=$ 7 , unpaired t test, $\mathrm{p}=0.0001$ ) in a similar manner as cocaine. However the dose is lower for nisoxetine, indicating nisoxetine has a higher affinity for DAT, as has been reported for in vitro studies (10). These studies indicate that the dopamine transporter is specifically responsible for dopamine uptake.

Slowed clearance time after uptake inhibition is consistent with results found in mammalian studies. Measurements in the rat striatum show that cocaine increases the time course of signal decay by $50 \%$ (39). Additionally, uptake inhibition is expected to increase the peak concentration, since no dopamine will be cleared during the stimulation. For example, brain slice measurements in the nucleus accumbens and striatum of rats have shown significant increases in maximal dopamine concentration after cocaine administration $(28,40)$. Peak height increases after cocaine have been also been attributed to diffusion from distant sites or release effects $(41,42)$. We observed a trend towards higher release after uptake inhibition, but observing a significant increase in Drosophila might require a large number of samples because of the biological variability between samples. Within subject studies might better elucidate these differences.

Thus, we repeated the experiments with nisoxetine in the same sample for comparison. For these experiments, a VNC was dissected in buffer and an initial $7 \mathrm{~s}$, blue-light stimulation performed. Then, nisoxetine was added to the Petri dish to provide a final concentration of $20 \mu \mathrm{M}$ and the same VNC stimulated at 15 min intervals. The 
example traces from the same-sample experiment show both an increase in peak height and decay time 30 min after nisoxetine application (Figure 2.6D). Evoked dopamine increased on average from $810 \pm 60 \mathrm{nM}$ to $960 \pm 50 \mathrm{nM}$, which is statistically significant $(\mathrm{n}=6$, paired t test, $\mathrm{p}=0.0046$, Figure 2.6E). This $20 \%$ increase is similar to increases in peak height after uptake inhibition observed in mammalian experiments (42). In samesample measurements, addition of nisoxetine significantly increased clearance time as well $(n=6$, paired t-test, $p=0.0005)$. The $t_{50}$ values from the separate group and same sample experiments are not significantly different $(\mathrm{p}=6$, unpaired t-test $\mathrm{p}=0.6943$, compare Figures 2.6 C and F). Therefore, the prior stimulation in the same sample experiment has no effect on the clearance time. These experiments demonstrate that our technique makes Drosophila amenable for studies of dopamine uptake inhibitors and drugs of abuse.

\subsection{7: Comparison to other techniques.}

We demonstrate the first direct, real-time measurement of endogenous dopamine release in a single Drosophila larva. Previously, HPLC and CE studies measured total dopamine tissue content in Drosophila, but provided no time course information about extracellular dopamine signaling $(13,14)$. Dopamine uptake has been measured in adult fruit flies by the Ewing group (18). They implanted a microelectrode in the protocerebral anterior medial brain area of an immobilized, intact adult fly. The time course of clearance of exogenously applied dopamine was measured using FSCV or amperometry. This experimental design provides information about uptake and they found that 
dopamine signaling was prolonged in mutant flies lacking DAT function or after administration of cocaine. Our data using cocaine in the larval CNS is consistent with their findings that dopamine uptake in Drosophila is slowed in the presence of a DAT inhibitor. For measuring uptake, our method and the Ewing method are complementary and both indicate Drosophila is a valid model system.

The primary advantage of our method is that we are able to evoke and detect endogenous dopamine release. The $\mathrm{ChR} 2$ stimulation is specific to one neuronal type and permits optical control of dopamine release. Our method will allow a range of experiments that test the effects of different pharmacological and genetic manipulations on dopamine release. For example, we could study exocytosis or the function of dopamine autoreceptors on the regulation of release. While it is not possible to make measurements in the intact larva because of body-wall contractions, our method takes advantage of whole CNS culture techniques that have been standardized in Drosophila. Therefore, neuronal connections are maintained, an advantage of using Drosophila larvae over mammalian brain slice experiments. 


\section{3: Conclusions}

In this chapter, I have shown that fast-scan cyclic voltammetry at carbon-fiber microelectrodes can be used to measure endogenous dopamine release evoked in an isolated Drosophila melanogaster larval ventral nerve cord. The release of dopamine measured in the presence of pharmacological agents in Drosophila is consistent with data collected from mammalian studies; therefore, dopaminergic signaling in Drosophila is homologous to signaling in mammals. Drosophila is a valuable model system to study dopamine regulation and the results could be useful for designing better mammalian experiments to reduce the use of higher order animals. For example, our method could be used to examine how chronic cocaine administration or cocaine withdrawal influences dopamine clearance in Drosophila. In the next chapter of this thesis, I use this method to study the function of dopamine2-like receptors, proving that this method is also useful for determining previously unknown neurobiology in Drosophila. Once dopamine regulation is better characterized in Drosophila, this system could be used to study flies with genetic mutations mimicking human diseases. 


\section{4: Experimental}

\subsection{1: Chemicals}

Unless otherwise noted, all chemicals were purchased from Sigma-Aldrich (St. Louis, MO) and used as received. All solutions were prepared using Milli-Q water (Millipore Billerica, MA). A modified Schneider's buffer (15.2 $\mathrm{mM} \mathrm{MgSO}_{4}, 21 \mathrm{mM}$ $\mathrm{KCl}, 3.3 \mathrm{mM} \mathrm{KH}_{2} \mathrm{PO}_{4}, 36 \mathrm{mM} \mathrm{NaCl}, 5.8 \mathrm{mM} \mathrm{NaH}_{2} \mathrm{PO}_{4}, 5.4 \mathrm{mM} \mathrm{CaCl}_{2}, 11.1 \mathrm{mM}$ glucose, $5.3 \mathrm{mM}$ trehalose, $\mathrm{pH}=6.2$ ) was used in all calibration experiments and dissections. $50 \mu \mathrm{M}$ cocaine and $20 \mu \mathrm{M}$ nisoxetine solutions were made using the modified Schneider's buffer. Similarly, a $100 \mu \mathrm{M}$ reserpine solution in buffer was made by dilution of a $10 \mathrm{mM}$ stock solution of reserpine dissolved in DMSO. A $20 \mu \mathrm{M}$ NSD1015 solution was made in water and mixed with the Drosophila food, Red Star yeast (Milwaukee, WI) mNSD-1015 concentration $6.6 \mu \mathrm{M}$. The drug-yeast mixture was fed to 3-day-old Drosophila larvae for 2 days prior to experimentation. Larvae were observed under a microscope and appeared healthy, but failed to show signs of pupation after 5 days.

\subsection{2: Electrochemical Measurements}

Carbon-fiber microelectrodes were fabricated by aspirating a single T-650 carbon fiber (Cytec Engineering Materials, West Patterson, NJ) into a $1.2 \mathrm{~mm}$ x $0.68 \mathrm{~mm}$ glass capillary (A-M systems, Carlsburg, WA). A vertical pipette puller (Narishige, model PE- 
21, East Meadow, NY) was used to form two electrodes. The carbon fiber was trimmed to a length of $40-60 \mu \mathrm{m}$ with a scalpel. In order to seal the fiber in the glass, the electrodes were epoxied with Epon Resin 828 (Miller-Stephenson, Danbury, CT) mixed with $14 \%$ (w/w) m-phenylenediamine hardener (Fluka, Milwaukee, WI). The epoxy and hardener were heated to approximately $85^{\circ} \mathrm{C}$ and the electrodes were dipped into the mixture for $30 \mathrm{~s}$ and allowed to dry overnight. They were then cured in an oven at $100^{\circ} \mathrm{C}$ for two hours followed by $150^{\circ} \mathrm{C}$ overnight. The electrodes were soaked in isopropanol for 10 minutes and backfilled with $1 \mathrm{M} \mathrm{KCl}$ prior to use.

A Dagan Chem-Clamp potentiostat with modified gain settings was used to collect data (Dagan, Minneapolis, MN). Tar Heel CV software (gift of Mark Wightman, University of North Carolina) was used for data collection and analysis. A homemade breakout box and 2 computer interface boards (PCI 6052 and PCI 6711, National Instruments, Austin TX) were used to apply the cyclic voltammogram waveform and to collect data as previously described (43). The electrode was scanned at a scan rate of 400 $\mathrm{V} / \mathrm{s}$ from -0.4 to $1.3 \mathrm{~V}$ and back at a repetition rate of $10 \mathrm{~Hz}$. $\mathrm{A} \mathrm{Ag} / \mathrm{AgCl}$ wire was used as a reference electrode and was placed in the buffer bath outside the nerve cord. Electrodes were calibrated with a $1 \mu \mathrm{M}$ dopamine solution before and after implantation into a VNC.

Statistics were performed using GraphPad Prism software. Data are reported as mean $+/$ - standard error of the mean (SEM) for $n$ number of different samples. Data were considered significantly different at the $95 \%$ confidence level. 


\subsection{3: Preparation of ventral nerve cords}

Flies containing UAS-ChR2 (a gift from Christian Schroll, Universitat Wurzburg) were crossed to flies expressing th-GAL4 (a gift from Jay Hirsh, University of Virginia) to generate homozygous lines with a th-GAL4; UAS-ChR2 genotype. Flies expressing thGALA were crossed with flies containing UAS-GFP (Bloomington, IN) to generate homozygous lines with a th-GAL4;UAS-GFP genotype. 3-day-old larvae were fed yeast containing a $1 \mathrm{mM}$ trans-retinal supplement for 2 days prior to dissection and were shielded from light. 5-day-old wandering $3^{\text {rd }}$ instar larvae were dissected in the modified Schneider's buffer unless otherwise noted. The entire CNS was removed and the optic lobes were removed by a horizontal cut across the anterior thorax region. The isolated ventral nerve cord (VNC) was adhered to the bottom of a plastic Petri dish (Becton Dickinson, Franklin Lakes, NJ) containing $4 \mathrm{~mL}$ of buffer. The dish was placed on a fluorescent microscope and a 40x water immersion lens was used to visualize the nerve cord while an electrode was inserted into the neuropil region using a micromanipulator. The electrode was inserted a distance of 4-5 segments away from the cut edge. For acute pharmacological experiments in different nerve cords, samples were dissected in the presence of drug and incubated for 20-30 minutes prior to electrode implantation to allow the drug to diffuse into the nerve cord. For experiments in the same nerve cord, a sample was dissected and placed in $3 \mathrm{~mL}$ modified Schneider's buffer, an electrode implanted and $1 \mathrm{~mL}$ of $80 \mu \mathrm{M}$ nisoxetine was added to the Petri dish to provide a final concentration of $20 \mu \mathrm{M}$ nisoxetine. 
All experiments were performed in a dark room. The electrode was allowed to equilibrate after implantation for at least 5 minutes before the initiation of data collection. Unless otherwise indicated, $30 \mathrm{~s}$ of baseline electrochemistry data was collected before VNCs were exposed to $7 \mathrm{~s}$ of blue-light illumination from a $10 \mathrm{~W}$ halogen microscope bulb with a standard fluorescein emission filter $(450-490 \mathrm{~nm})$. Stimulations were repeated at 15 minute intervals to allow for recovery.

\subsection{4: Immunohistochemistry Experiments}

Flies expressing green fluorescent protein (GFP) in their dopaminergic neurons (th-GALA;UAS-GFP) were mounted and stained using anti-chicken GFP polyclonal antibodies (Aves Labs, Tigard, Oregon) (44). Dopamine cell bodies were imaged with an Olympus 1x70 microscope with a Fluoview 3.3 laser scanning confocal system (Olympus America, Melville, NY). Image reconstitutions were performed with Volocity 2.0 (Improvision, Waltham, MA). 


\section{5: Reference List}

1. Collins, C. A., and DiAntonio, A. (2007) Synaptic development: insights from Drosophila, Curr. Opin. Neurobiol. 17, 35-42.

2. Prokop, A., and Meinertzhagen, I. A. (2006) Development and structure of synaptic contacts in Drosophila, Semin. Cell Dev. Biol. 17, 20-30.

3. Olsen, S. R., and Wilson, R. I. (2008) Cracking neural circuits in a tiny brain: new approaches for understanding the neural circuitry of Drosophila, Trends Neurosci. $31,512-520$.

4. Watson, M. R., Lagow, R. D., Xu, K., Zhang, B., and Bonini, N. M. (2008) A Drosophila Model for Amyotrophic Lateral Sclerosis Reveals Motor Neuron Damage by Human SOD1, J. Biol. Chem. 283, 24972-81.

5. Finelli, A., Kelkar, A., Song, H. J., Yang, H., and Konsolaki, M. (2004) A model for studying Alzheimer's A $\beta 42$-induced toxicity in Drosophila melanogaster, Mol. Cell Neurosci. 26, 365-375.

6. Feany, M. B., and Bender, W. W. (2000) A Drosophila model of Parkinson's disease, Nature 404, 394-398.

7. Rubin, G. M., Yandell, M. D., Wortman, J. R., Gabor Miklos, G. L., Nelson, C. R., Hariharan, I. K., Fortini, M. E., Li, P. W., Apweiler, R., Fleischmann, W., Cherry, J. M., Henikoff, S., Skupski, M. P., Misra, S., Ashburner, M., Birney, E., Boguski, M. S., Brody, T., Brokstein, P., Celniker, S. E., Chervitz, S. A., Coates, D., Cravchik, A., Gabrielian, A., Galle, R. F., Gelbart, W. M., George, R. A., Goldstein, L. S., Gong, F., Guan, P., Harris, N. L., Hay, B. A., Hoskins, R. A., Li, J., Li, Z., Hynes, R. O., Jones, S. J., Kuehl, P. M., Lemaitre, B., Littleton, J. T., Morrison, D. K., Mungall, C., O'Farrell, P. H., Pickeral, O. K., Shue, C., Vosshall, L. B., Zhang, J., Zhao, Q., Zheng, X. H., and Lewis, S. (2000) Comparative Genomics of the Eukaryotes, Science 287, 2204-2215.

8. Bier, E. (2005) Drosophila, the golden bug, emerges as a tool for human genetics, Nat Rev Genet 6, 9-23.

9. Neckameyer, W., Holt, B., and Paradowski, T. (2005) Biochemical Conservation of Recombinant Drosophila Tyrosine Hydroxylase with its Mammalian Cognates, Biochem. Genet. 43, 425-443.

10. Porzgen, P., Park, S. K., Hirsh, J., Sonders, M. S., and Amara, S. G. (2001) The Antidepressant-Sensitive Dopamine Transporter inDrosophila melanogaster: A Primordial Carrier for Catecholamines, Mol. Pharmacol. 59, 83-95. 
11. Hsouna, A., Lawal, H. O., Izevbaye, I., Hsu, T., and O’Donnell, J. M. (2007) Drosophila dopamine synthesis pathway genes regulate tracheal morphogenesis, Dev. Biol. 308, 30-43.

12. Budnik, V., and White, K. (1988) Catecholamine-containing neurons in Drosophila melanogaster: Distribution and development, J. Comp. Neurol. 268, 400-413.

13. Paxon, T. L., Powell, P. R., Lee, H.-G., Han, K.-A., and Ewing, A. G. (2005) Microcolumn Separation of Amine Metabolites in the Fruit Fly, Anal. Chem. 77, 5349-5355.

14. Hardie, S. L., and Hirsh, J. (2006) An improved method for the separation and detection of biogenic amines in adult Drosophila brain extracts by high performance liquid chromatography, Journal of Neuroscience Methods 153, 243 249.

15. Dasari, S., Viele, K., Turner, A. C., and Cooper, R. L. (2007) Influence of PCPA and MDMA (ecstasy) on physiology, development and behavior in Drosophila melanogaster, European Journal of Neuroscience 26, 424-438.

16. Wightman, R. M., Amatorh, C., Engstrom, R. C., Hale, P. D., Kristensen, E. W., Kuhr, W. G., and May, L. J. (1988) Real-time characterization of dopamine overflow and uptake in the rat striatum, Neuroscience 25, 513-523.

17. Robinson, D. L., Venton, B. J., Heien, M. L. A. ., and Wightman, R. M. (2003) Detecting Subsecond Dopamine Release with Fast-Scan Cyclic Voltammetry in Vivo, Clin Chem 49, 1763-1773.

18. Makos, M. A., Kim, Y.-C., Han, K.-A., Heien, M. L., and Ewing, A. G. (2009) In Vivo Electrochemical Measurements of Exogenously Applied Dopamine in Drosophila melanogaster, Anal. Chem. 81, 1848-1854.

19. Borue, X., Cooper, S., Hirsh, J., Condron, B., and Venton, B. J. (2009) Quantitative evaluation of serotonin release and clearance in Drosophila, J. Neurosci. Methods 179, 300-308.

20. McGuire, S. E., Roman, G., and Davis, R. L. (2004) Gene expression systems in Drosophila: a synthesis of time and space, Trends Genet. 20, 384-391.

21. Schroll, C., Riemensperger, T., Bucher, D., Ehmer, J., Völler, T., Erbguth, K., Gerber, B., Hendel, T., Nagel, G., Buchner, E., and Fiala, A. (2006) LightInduced Activation of Distinct Modulatory Neurons Triggers Appetitive or Aversive Learning in Drosophila Larvae, Current Biology 16, 1741-1747. 
22. Vickrey, T. L., Condron, B., and Venton, B. J. (2009) Detection of Endogenous Dopamine Changes in Drosophila melanogaster Using Fast-Scan Cyclic Voltammetry, Analytical Chemistry 81, 9306-9313.

23. Wright, T. R. (1987) The Genetics Of Biogenic Amine Metabolism, Sclerotization, And Melanization In Drosophila Melanogaster, Adv.Genet. 24, 127-222.

24. Neckameyer, W. S. (1998) Dopamine modulates female sexual receptivity in Drosophila melanogaster, Journal of Neurogenetics 12, 101-114.

25. McClung, C., and Hirsh, J. (1998) Stereotypic behavioral responses to free-base cocaine and the development of behavioral sensitization in Drosophila, Curr. Biol. 8, 109-112.

26. Torres, G., and Horowitz, J. M. (1998) Activating properties of cocaine and cocaethylene in a behavioral preparation of Drosophila melanogaster, Synapse 29, $148-61$.

27. Sykes, P. A., and Condron, B. G. (2005) Development and sensitivity to serotonin of Drosophila serotonergic varicosities in the central nervous system, Dev. Biol. 286, 207-216.

28. Jones, S. R., Garris, P. A., Kilts, C. D., and Wightman, R. M. (1995) Comparison of Dopamine Uptake in the Basolateral Amygdaloid Nucleus, Caudate-Putamen, and Nucleus Accumbens of the Rat, J. Neurochem. 64, 2581-2589.

29. Budygin, E. A., John, C. E., Mateo, Y., and Jones, S. R. (2002) Lack of cocaine effect on dopamine clearance in the core and shell of the nucleus accumbens of dopamine transporter knock-out mice, J. Neurosci. 22, RC222.

30. Garris, P. A., Ciolkowski, E. L., Pastore, P., and Wightman, R. M. (1994) Efflux of dopamine from the synaptic cleft in the nucleus accumbens of the rat brain, $\mathrm{J}$. Neurosci. 14, 6084-6093.

31. Phillips, P. E. M., Stuber, G. D., Heien, M. L. A. V., Wightman, R. M., and Carelli, R. M. (2003) Subsecond dopamine release promotes cocaine seeking, Nature 422, 614-618.

32. Cheer, J. F., Wassum, K. M., Heien, M. L., Phillips, P. E., and Wightman, R. M. (2004) Cannabinoids Enhance Subsecond Dopamine Release in the Nucleus Accumbens of Awake Rats, J. Neurosci. 24, 4393-4400. 
33. Li, X., Gutierrez, D. V., Hanson, M. G., Han, J., Mark, M. D., Chiel, H., Hegemann, P., Landmesser, L. T., and Herlitze, S. (2005) Fast noninvasive activation and inhibition of neural and network activity by vertebrate rhodopsin and green algae channelrhodopsin, Proc. Natl. Acad. Sci. U.S.A 102, 1781617821.

34. Bath, B. D., Michael, D. J., Trafton, B. J., Joseph, J. D., Runnels, P. L., and Wightman, R. M. (2000) Subsecond Adsorption and Desorption of Dopamine at Carbon-Fiber Microelectrodes, Anal. Chem. 72, 5994-6002.

35. Phillips, P. E., and Wightman, R. M. (2003) Critical guidelines for validation of the selectivity of in-vivo chemical microsensors, Trends Anal. Chem. 22, 509514.

36. Rice, M. E., Richards, C. D., Nedergaard, S., Hounsgaard, J., Nicholson, C., and Greenfield, S. A. (1994) Direct monitoring of dopamine and 5-HT release in substantia nigra and ventral tegmental area in vitro, Exp. Brain Res. 100, 395406.

37. Chang, H. Y., Grygoruk, A., Brooks, E. S., Ackerson, L. C., Maidment, N. T., Bainton, R. J., and Krantz, D. E. (2006) Overexpression of the Drosophila vesicular monoamine transporter increases motor activity and courtship but decreases the behavioral response to cocaine, Mol. Psychiatry 11, 99-113.

38. Greco, P. G., and Garris, P. A. (2003) In vivo interaction of cocaine with the dopamine transporter as measured by voltammetry, Eur. J. Pharmacol. 479, 117125.

39. Zahniser, N. R., Larson, G. A., and Gerhardt, G. A. (1999) In vivo dopamine clearance rate in rat striatum: regulation by extracellular dopamine concentration and dopamine transporter inhibitors, J. Pharmacol. Exp. Ther. 289, 266-277.

40. Jones, S. R., Garris, P. A., and Wightman, R. M. (1995) Different effects of cocaine and nomifensine on dopamine uptake in the caudate-putamen and nucleus accumbens., J. Pharmacol. Exp. Ther. 274, $396-403$.

41. Hafizi, S., Palij, P., and Stamford, J. A. (1992) Activity of two primary human metabolites of nomifensine on stimulated efflux and uptake of dopamine in the striatum: In vitro voltammetric data in slices of rat brain, Neuropharmacology 31, 817-824.

42. Venton, B. J., Seipel, A. T., Phillips, P. E., Wetsel, W. C., Gitler, D., Greengard, P., Augustine, G. J., and Wightman, R. M. (2006) Cocaine Increases Dopamine 
Release by Mobilization of a Synapsin-Dependent Reserve Pool, J. Neurosci. 26, 3206-3209.

43. Heien, M. L. A. V., Phillips, P. E. M., Stuber, G. D., Seipel, A. T., and Wightman, R. M. (2003) Overoxidation of carbon-fiber microelectrodes enhances dopamine adsorption and increases sensitivity, Analyst 128, 1413-1419.

44. Daubert, E. A., and Condron, B. G. (2007) A Behavioral Stress Assay in Drosophila Larvae, Cold Spring Harbor Protoc. db. 


\section{Chapter 3}

\section{Drosophila Dopamine2-like receptors function as autoreceptors}

"The saddest aspect of life right now is that science gathers knowledge faster than society gathers wisdom."

$\sim$ Isaac Asimov 


\title{
Chapter 3: Drosophila Dopamine2-like receptors function as autoreceptors
}

\author{
Abstract \\ Dopaminergic signaling pathways are conserved between mammals and \\ Drosophila and D2 receptors have been identified in Drosophila. However, it has not \\ been demonstrated whether Drosophila D2 receptors function as autoreceptors and \\ regulate the release of dopamine. In this chapter of my thesis, I determine if Drosophila \\ D2 receptors act as autoreceptors by probing the extent to which D2 agonists and \\ antagonists affect evoked dopamine release. Fast-scan cyclic voltammetry was used to \\ measure stimulated dopamine release at a carbon-fiber microelectrode implanted in an \\ intact, larval Drosophila nervous system. Dopamine release was evoked using 5-second \\ blue light stimulations that open Channelrhodopsin-2, a blue light activated cation \\ channel that was specifically expressed in dopaminergic neurons. In mammals, \\ administration of a D2 agonist decreases evoked dopamine release by increasing \\ autoreceptor feedback. Similarly, we found that the D2 agonists bromocriptine and \\ quinpirole decreased stimulated dopamine release in Drosophila. D2 antagonists were \\ expected to increase dopamine release and the D2 antagonists flupenthixol, butaclamol, \\ and haloperidol did increase stimulated release. However, another D2 antagonist, \\ raclopride, decreased evoked release but increased basal dopamine levels. The effect of \\ raclopride on basal levels was not as large in genetic mutants underexpressing D2 \\ receptors (th-Gal4;UAS-ds-DD2R), demonstrating that the effect is mediated by D2 \\ receptors. In addition, agonists did not significantly modulate dopamine uptake although \\ the modulatory effects of D2 drugs on release were affected by prior administration of the
}


uptake inhibitor nisoxetine. These results demonstrate that the $\mathrm{D} 2$ receptor functions as an autoreceptor in Drosophila. The similarities in dopamine regulation validate Drosophila as a model system for studying the basic neurobiology of dopaminergic signaling. 


\subsection{Introduction}

The monoamine neurotransmitter dopamine plays a major role in many human behaviors such as movement, cognition, reward, addiction, and motivation. Abnormalities in dopaminergic signaling are implicated in diseases such as schizophrenia, Parkinson's disease, and drug addiction. Dopaminergic signaling is mediated by receptors that are located either postsynaptically, where they regulate downstream signaling, or presynaptically, where they act as autoreceptors regulating release (1). D2 receptors (D2Rs) are the predominant dopamine autoreceptor and dysfunction of D2 autoreceptors is involved in disease etiology (2). Therefore, D2 receptors are important drug target sites (2). For example, patients with schizophrenia have a higher expression of D2 receptors and higher basal levels of dopamine; thus many antipsychotics target the D2 receptor (3). Other studies have shown that mice without the D2R gene have significant neurological impairments and Parkinson-like symptoms (4). Consequently, D2Rs are targets for Parkinson treatment (5). In addition to their implication in specific diseases, D2Rs have also been shown to modulate locomotion ( 6 , 7). Thus, autoreceptors are critical for regulating dopamine release and maintaining dopaminergic function.

Drosophila is a popular biological model system because of its short life span, high fecundity, and facile genetics. Genetic mutants mimicking human diseases can be constructed and studied more rapidly in Drosophila than in mammals. Cellular machinery that controls dopamine regulation, such as transporter proteins, synthesis enzymes, and vesicles are conserved between Drosophila and mammals $(8,9)$. Our lab has recently 
developed a method to directly measure dopamine release in Drosophila and has verified that release and reuptake rates are similar to mammals $(10,11)$. However, the extent to which dopamine receptors in Drosophila act as autoreceptors has not been tested.

Three mammalian isoforms of D2R, differing by up to 29 amino acids, have been isolated: the D2 short $\left(\mathrm{D} 22_{\mathrm{S}}\right)$, D2 long $\left(\mathrm{D} 2_{\mathrm{L}}\right)$, and $\mathrm{D} 2$ extra long $(12,13)$. The $\mathrm{D} 2 \mathrm{~S}$ receptor subtype is located presynaptically and functions as an autoreceptor, while the $\mathrm{D} 2_{\mathrm{L}}$ is located postsynaptically (14). Both isoforms are found in many species: human, rat, mouse, bovine, c. elegans, and xenopus (15-19). Eight isoforms of a Drosophila D2-like receptor (DD2R) have been identified. These DD2Rs are G-protein coupled receptors with a high affinity for dopamine that have homologous amino acid sequences to mammalian D2-like receptors (20). It is unclear whether these receptors are $\mathrm{D} 2 \mathrm{~L}$ or $\mathrm{D} 2 \mathrm{~S}$ and identifying the cellular locations and function of these DD2R receptors is difficult. Immunohistochemistry studies have identified DDR2 localization in larva and DD2R staining is co-localized with both dopaminergic cell bodies and projections, although the expression presynaptically or postsynaptically has not been determined (21). DD2Rs were expressed in HEK293 cells and pharmacological evaluation with mammalian D2R agonists and antagonists showed that the agonist bromocriptine and the antagonists flupenthixol and butaclamol had high affinity binding. In contrast, the agonist quinpirole and the antagonist haloperidol had little to no affinity for the DD2Rs (20). However, some drugs with poor affinity cause behavioral effects in Drosophila. For example, the agonist quinpirole increases locomotor activity in adults $(22,23)$. Another tool to study DDR2 function is an RNA interference (RNAi) Drosophila mutant with decreased 
expression of DD2R (th-GAL4;UAS-ds-DD2R) (21). These mutants exhibit decreases in locomotor response, which can be rescued by the agonist bromocriptine (21). Molecular biology, behavioral, and genetic results suggest that $\mathrm{D} 2$ autoreceptor functionality may be conserved in Drosophila. Chemical measurements of dopamine release would provide direct evidence and establish the relative effectiveness of DD2R drugs in an intact Drosophila CNS.

In this study, we used fast-scan cyclic voltammetry at carbon-fiber microelectrodes to characterize changes in evoked dopamine release in Drosophila larvae following pharmacological manipulation with different D2 agonists and antagonists. We show D2 agonists decreased stimulated dopamine release and D2 antagonists increased dopamine release. While the antagonist raclopride did not increase stimulated release, it did increase basal dopamine levels, an effect that was reduced in flies under-expressing DD2Rs (th-Gal4;UAS-ds-DD2R). These studies demonstrate that the Drosophila D2 receptor functions as an autoreceptor and regulates dopamine release, thus validating Drosophila as a model system to study dopaminergic diseases. This chapter is adapted from my published work in ACS Chemical Neuroscience (24). 


\section{2: Results and Discussion}

\subsection{1: Overview}

Fast-scan cyclic voltammetry (FSCV) at carbon-fiber microelectrodes has been used extensively to measure electrically-stimulated dopamine release in animal models in vivo, in brain slices, and at single cells (25-27). In mammals, FSCV has been used to measure the effects of D2 receptor agonists and antagonists on evoked dopamine release $(28,29)$. For example, in rat brain slices, the dopamine agonist quinpirole decreases stimulated dopamine release (29) and in anesthetized rats, the dopamine antagonist flupenthixol increases stimulated dopamine release (30). The combination of specific stimulation of the dopaminergic terminals and rapid measuring technique allow presynaptic effects of the drugs to be probed so these studies verify that D2 receptors act as autoreceptors, regulating a feedback loop that controls release (31). While electrical stimulation works well in mammalian experiments, the Drosophila ventral nerve cord is smaller than a typical stimulating electrode. Therefore, optical stimulations are used instead of electrical stimulations. Channelrhodopsin-2, a blue-light sensitive ion channel, is specifically expressed in dopaminergic neurons. Dopamine release is measured with FSCV at a carbon-fiber microelectrode implanted in an isolated Drosophila larva nerve cord after blue-light stimulation $(10,11)$. Presynaptic effects of the drugs are investigated as this experimental protocol is analogous to the mammalian protocols because dopaminergic terminals are specifically activated and release measured 
on a rapid time scale (31). The effects of D2R agonists and antagonists were tested on ChR2-mediated dopamine release to determine autoreceptor function.

\subsection{2: Dopamine agonists decrease evoked dopamine release}

In humans, bromocriptine is a potent D2R agonist used in the treatment of Parkinson's disease. Administering bromocriptine to mammals decreases striatal dopamine synthesis through activation of the D2R (32). In Drosophila, bromocriptine restores locomotion to mutants exhibiting Parkinsonian-like behavior (21). Bromocriptine had a similar affinity for Drosophila D2R (DD2R) as for human D2R and has the highest affinity binding of several common dopamine receptor agonists for DDR2 receptors transfected into HEK293 cells (33).

To test the effects of drugs in Drosophila, an electrode was implanted into a ventral nerve cord dissected from a 5-day-old larva and an initial, $5 \mathrm{~s}$ blue-light stimulation performed to assess dopamine release before drug administration. Two, subsequent $5 \mathrm{~s}$ blue-light stimulations were performed 15 and $30 \mathrm{~min}$ after drug administration. Figure 3.1 A shows that in a control experiment where buffer is added instead of drugs, the stimulations after 15 and 30 min produced the same dopamine signal as the initial stimulation. While there was a large variation in release between samples due to variance in ChR2 expression (average evoked dopamine release was $490 \pm 60 \mathrm{nM}$ for all flies, $n=42$ ), stimulated release at 15 min intervals within each sample was stable.

Figures 3.1 B and C show example cyclic voltammograms and concentration versus time profiles for evoked release in the fly before and after bromocriptine was 
administered to bring the concentration in the bath around the nerve cord to $50 \mu \mathrm{M}$. Peak concentration decreased and there was a larger effect 30 min (47\% decrease) after bromocriptine than after $15 \mathrm{~min}$ (24\% decrease). Pooled data are plotted as a percentage of the initial stimulation (Figure 3.1 D) and bromocriptine decreased evoked dopamine release. To test significance, evoked release before and after drug were compared with a paired t-test and release was significantly decreased both 15 and 30 min postdrug.

A. Control

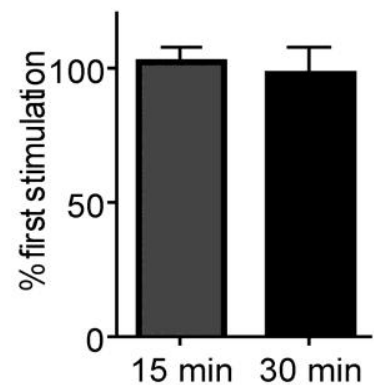

C. Bromocriptine Conc. vs time

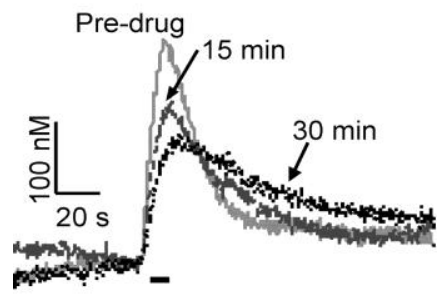

\section{E. Incubated} in Bromocriptine

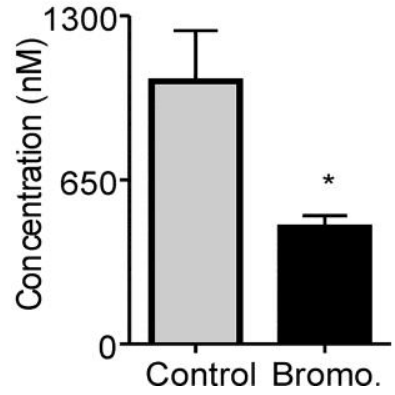

B. Bromocriptine CVs

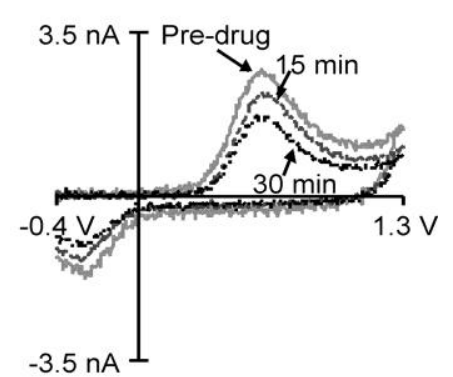

D. Bromocriptine Averages

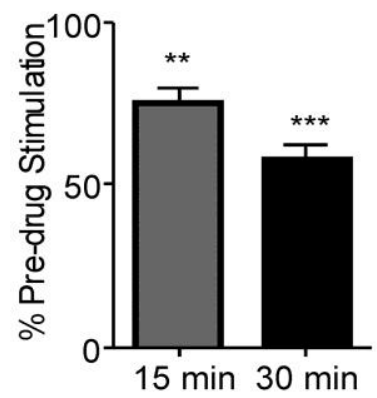

F. Bromocriptine Clearance

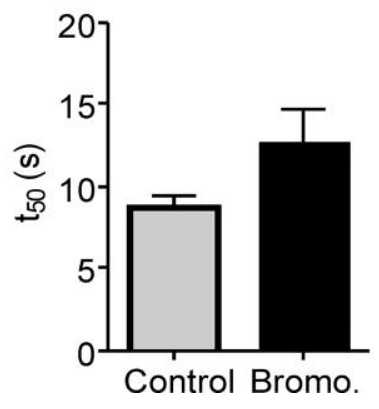

Figure 3.1: Effect of D2 agonist bromocriptine on evoked dopamine. (A) Control data show that when buffer is added instead of drug, stimulations evoked 15 and $30 \mathrm{~min}$ after the initial stimulation are stable $(n=5)$. (B) Background-subtracted cyclic voltammogram from a single nerve cord comparing evoked dopamine release before the addition of drug and 15 and $30 \mathrm{~min}$ after application of $50 \mu \mathrm{M}$ bromocriptine. (C) Concentration vs time profile showing the effect of bromocriptine on stimulated dopamine release in a single nerve cord. The bar underneath marks the duration of the blue-light stimulation. (D) Pooled data $(n=7)$ show $50 \mu \mathrm{M}$ bromocriptine decreased evoked dopamine release. Data are normalized to the initial stimulation in each animal. Statistics were determined via comparison of evoked release before and after addition of drug using paired $t$ tests. $* * p<0.01 ; * * * p<0.001$. (E) Preincubation with $50 \mu \mathrm{M}$ bromocriptine also decreased evoked dopamine release $(n=6)$. Significance is determined using an unpaired $t$ test. $* p<0.05$. (F) Dopamine clearance $(t 50)$ is not significantly different in the presence of bromocriptine (paired $t$ test; $n=6$ ). 
In order to ensure that pre-stimulation did not deplete the population of dopamineloaded vesicles or change synaptic physiology, nerve cords were also incubated with bromocriptine 15-20 minutes before any stimulations were performed. Figure 1E shows evoked dopamine release is significantly less after bromocriptine incubation than in control samples. The effect of bromocriptine is the same regardless of prior stimulation, although the decrease in release (57\%) is slightly larger. The larger decrease after incubation could be due to the experimental protocol facilitating more bromocriptine diffusion, as the optic lobes were removed in bromocriptine and more drug could have entered during the cutting when no glial barrier would have formed. However, the concentration of stimulated release after bromocriptine was about the same for both the pre-stimulated and unstimulated groups. The control data for this experiment had larger than average release so these data show why it is useful to do pre-stimulation to be able to directly compare drug to control data in the same sample.

In addition to evoked concentration, dopamine clearance kinetics can be determined from current vs. time plots. The time from the end of the stimulus until the signal decays to the half maximal concentration is reached, $\mathrm{t}_{50}$, can be used as an estimate of uptake by the dopamine transporter. The $\mathrm{t}_{50}$ increased slightly for nerve cords after bromocriptine (Figure 3.1 F), although the effect was not significant. 
The D2R agonist quinpirole

was also tested. In mammalian brain

slice experiments, quinpirole

significantly decreases the amount of

stimulated dopamine release, while in

behaving rats quinpirole modulates

locomotion in a concentration

dependent manner $(21,34)$. In

Drosophila, administration of

quinpirole to the nerve cord of

decapitated adult flies stimulates

locomotion and grooming responses

(22). However, quinpirole had no

significant affinity for DD2R in cells

transfected with Drosophila D2R

isoforms (20). Fifty $\mu \mathrm{M}$ quinpirole was

\section{A. Quinpirole conc. vs time}

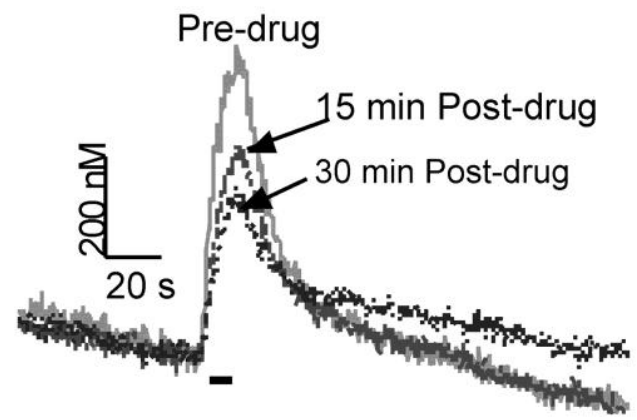

B. Release

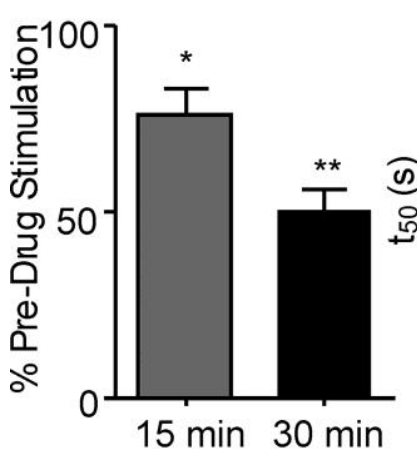

C. Clearance

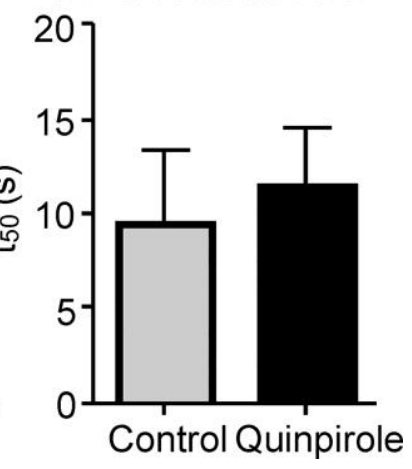

Figure 3.2: Effect of D2 agonist quinpirole. (A) Concentration-time traces show $50 \mu \mathrm{M}$ quinpriole decreased evoked dopamine release. (B) Averaged data demonstrate $(n=7)$ evoked release is significantly decreased 15 and 30 min after addition of quinpirole (paired $t$ test comparing evoked release before and after addition of drug). (C) Dopamine clearance $(t 50)$ is not significantly reduced in the presence of quinpirole (paired $t$ test; $n=6$ ). ${ }^{*} p<$ $0.05 . * * p<0.01$.

administered, the same dose for

bromocriptine and similar to that used in behavioral experiments $(22,29,35)$. Figure 3.2 shows that stimulated dopamine release decreased after quinpirole administration. On average release was 35\% and $69 \%$ less 15 and 30 min after quinpirole, respectively, which is consistent with mammalian results $(36,37)$. We observed a significant effect on evoked release with quinpirole, whereas significant binding was not seen in transfected 
cells (20). It is common for drug binding in vivo to be different than in transfected cells; thus, an in vivo method to test drugs is valuable $(38,39)$. For quinpirole, the $t_{50}$ did not significantly change (Figure $3.2 \mathrm{C}$ ), and the trend towards a higher $\mathrm{t}_{50}$ was similar to that observed with bromocriptine.

In summary, both D2R agonists bromocriptine and quinpirole significantly decreased dopamine release. This decrease in release suggests that the Drosophila D2R functions as an autoreceptor, regulating dopamine release.

\subsection{3: Dopamine antagonists increase evoked dopamine release}

If the DD2R is an autoreceptor, then D2 antagonists should increase stimulated dopamine release. Flupenthixol is a D2 antagonist used to treat schizophrenia (40). Acute flupenthixol upregulates dopamine synthesis in mammals (41) and increases stimulated dopamine release in rats (30). In HEK293 cells transfected with DD2R isoforms, flupenthixol showed the highest affinity among antagonists tested (20). Figure 3.3 A shows an example concentration versus time profile for evoked dopamine after 5 $\mu \mathrm{M}$ flupenthixol. The highest release was observed 15 min after antagonist administration, although release was still elevated after 30 min. Figure $3.3 \mathrm{~B}$ demonstrates that on average endogenous dopamine release doubled 15 minutes after flupenthixol administration, a significant increase. The increase in dopamine release after flupenthixol is similar to mammalian studies and is consistent with the DD2R acting as an autoreceptor. The $\mathrm{t}_{50}$ increased significantly for nerve cords after flupenthixol 
(Figure 3.3 B). Flupenthixol is a weak human dopamine transporter inhibitor; therefore, it is not surprising that clearance was significantly decreased (42).

A. Flupenthixol

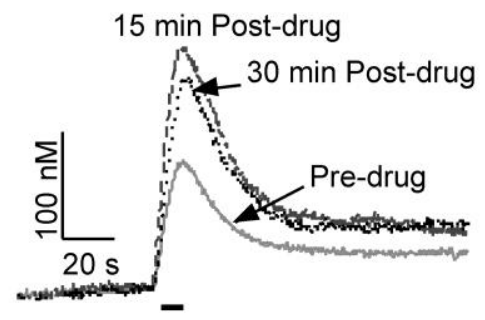

C. Butaclamol

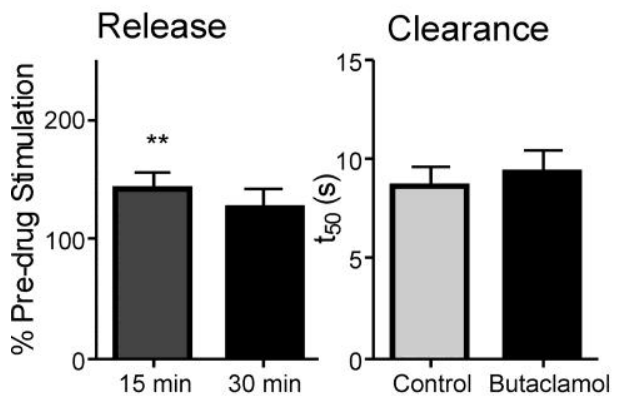

B. Flupenthixol
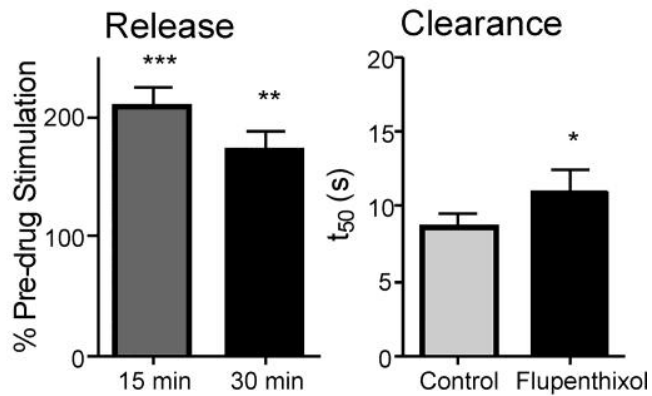

D. Haloperidol

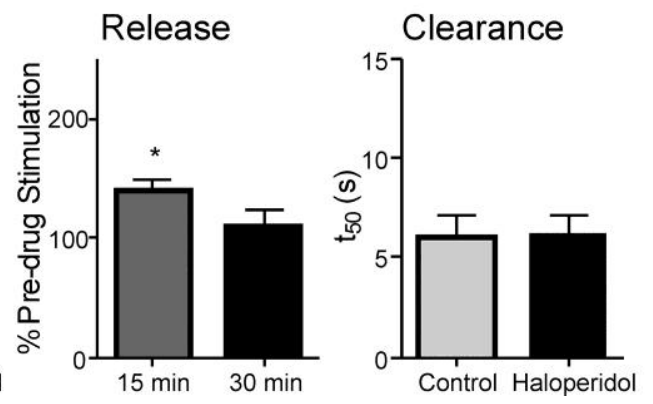

Figure 3.3: Figure 3.3: Effect of dopamine D2 antagonists. (A) An example concentration-time profile shows $5 \mu \mathrm{M}$ flupenthixol increased dopamine release. Release is higher $15 \mathrm{~min}$ than $30 \mathrm{~min}$ after addition of drug. (B) Averaged data for $5 \mu \mathrm{M}$ flupenthixol show that release was significantly increased 15 and 30 min after addition of drug and that the time for dopamine clearance significantly increases after addition of $5 \mu \mathrm{M}$ flupenthixol $(n=6)$. (C) Averaged data for $5 \mu \mathrm{M}$ butaclamol show that evoked release is significantly increased $15 \mathrm{~min}$ after addition of drug but not $30 \mathrm{~min}$, and $t_{50}$ is not significantly different $(n=5)$. (D) Averaged data for $5 \mu \mathrm{M}$ haloperidol show a significant increase in release after $15 \mathrm{~min}$ and no change in $t_{50}(n=6)$. All statistics are from paired $t$ tests of release or clearance before and after addition of drug. ${ }^{*} p<0.05$. $* * p<0.01 . * * * p<0.001$.

Butaclamol is another D2R antagonist that was developed to treat schizophrenia,

but showed a high incidence of extrapyramidal side effects and was never marketed (43).

In mammalian models, butaclamol has a high affinity for D2 receptors and in DDR2-

transfected cells, butaclamol has a significant affinity for DD2R $(20,44)$. Butaclamol

significantly increased stimulated dopamine release $15 \mathrm{~min}$ after $5 \mu \mathrm{M}$ was administered 
but not after 30 min (Figure $3.3 \mathrm{C}$ ). The $\mathrm{t}_{50}$ was not significantly different after butaclamol (Figure 3.3 C).

Haloperidol is a common antipsychotic used in humans, with significant affinity for D2R (45). Haloperidol has been used extensively in mammalian autoreceptor studies (20). For example, haloperidol increases electrically-evoked dopamine release in freelymoving animals $(46,47)$. In cells transfected with DD2R, haloperidol had a lower affinity than flupenthixol and butaclamol (20). Evoked dopamine release was significantly increased $15 \mathrm{~min}$ after $5 \mu \mathrm{M}$ haloperidol but not at $30 \mathrm{~min}$ (Figure $3.3 \mathrm{D}$ ). Figure $3.3 \mathrm{D}$ shows that $\mathrm{t}_{50}$ did not significantly change after haloperidol.

The increase in stimulated dopamine release after flupenthixol, butaclamol, or haloperidol provides further evidence that the DD2Rs act as autoreceptors and that Drosophila is a homologous model system to mammals. The similarity of the response in Drosophila and mammals suggests that while the D2 receptor isoforms may be different, basic biology is conserved. Interestingly, the effect of the D2 antagonist was greatest after $15 \mathrm{~min}$. This could be caused by D2-mediated increases in basal dopamine levels, which could deplete the pool for stimulated release. Another cause for the greater effect at 15 min could be that receptor density is increased after prolonged exposure to the antagonist, which is observed in mammals on a longer time scale $(48,49)$. Flupenthixol had the greatest effect of all antagonists tested, consistent with flupenthixol having the highest affinity for DD2R in transfected cells (20). Butaclamol and haloperidol produced very similar results, even though haloperidol had a lower affinity for DDR2 in transfected cells. Thus, electrochemical detection of dopamine release in 
Drosophila provides an easy test for pharmacological efficacy of D2 agonists and antagonists.

\subsection{4: Raclopride increases basal dopamine levels}

Raclopride is a potent D2 antagonist in mammals, and in vivo voltammetry studies in rats have shown raclopride increases the concentration of evoked dopamine (50). Raclopride also attenuates locomotor activity in rats (51). In Drosophila, raclopride administration to nerve cords of decapitated adult flies also decreased locomotor activity (22). However, raclopride exhibited little to no affinity for the Drosophila D2 receptor in transfected cells (20). Figure 3.4 A shows a concentration vs. time profile for stimulated release before and after $5 \mu \mathrm{M}$ raclopride. While the other antagonists increased stimulated dopamine, evoked dopamine after raclopride decreased. Figure 4 B shows that average release significantly decreased both 15 and 30 min after raclopride. A closer look at the raclopride concentration vs time traces reveals an interesting observation. The signal doesn't decay back to baseline after raclopride and a CV taken after stimulated dopamine is cleared has characteristics of dopamine, suggesting additional dopamine is released (Figure $3.4 \mathrm{~A}$ inset).

Previous in vivo studies in rats using differential pulse voltammetry have shown that raclopride can increase basal dopamine levels in the nucleus accumbens $190 \%$ in 30 minutes (50). Thus, to investigate changes in basal levels, we repeated our experiment by continuously recording voltammetry data before and after $5 \mu \mathrm{M}$ raclopride. Figure $3.4 \mathrm{C}$ shows a non-background subtracted current versus time profile from before the initial 


\section{A. Raclopride}

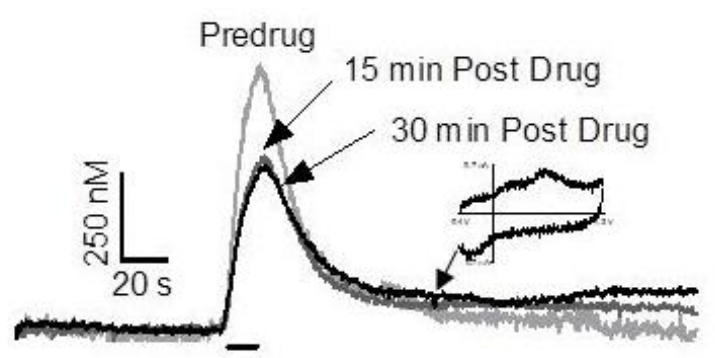

\section{Raclopride-Drosophila}

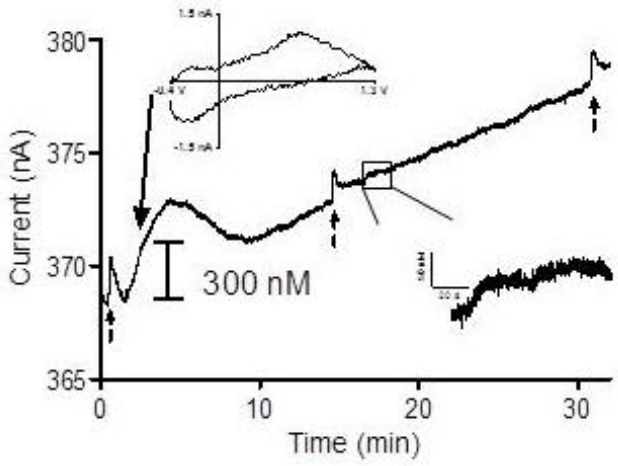

\section{E. Ralcopride - DD2R Mutants}
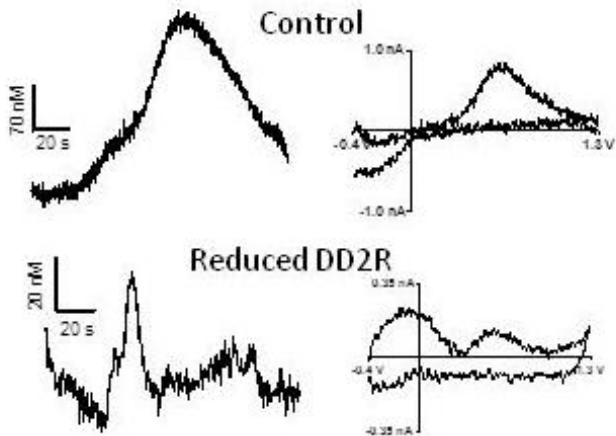

\section{B. Raclopride}

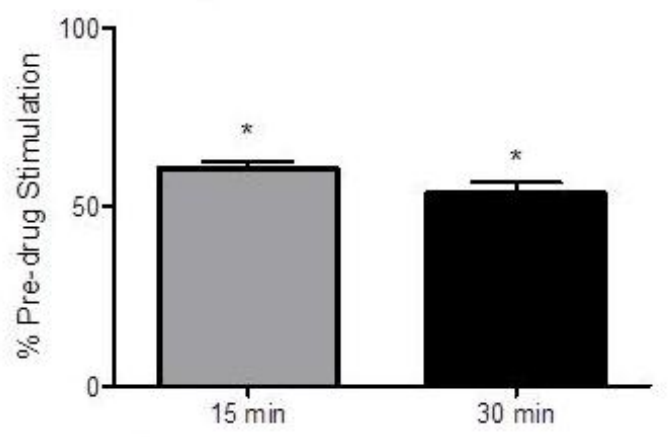

D. Raclopride-Rat

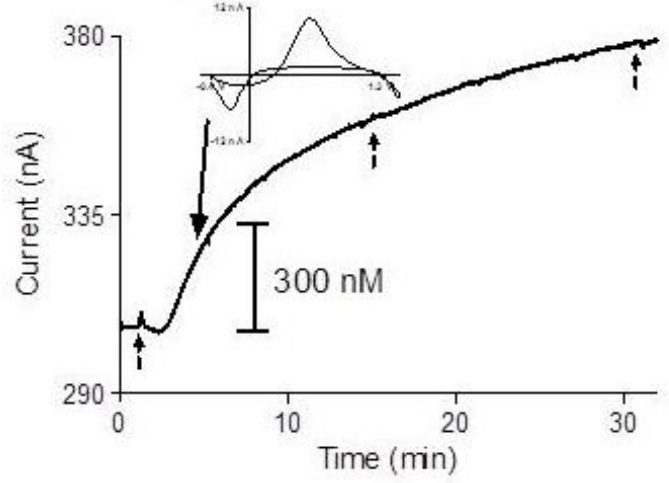

F. DA Events in $20 \mathrm{~min}$

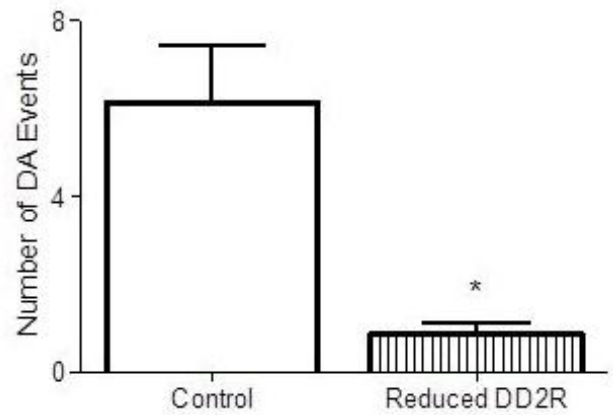

Figure 3.4: Effect of raclopride. A) Concentration vs. time profile of stimulated release before and after 5 $\mu \mathrm{M}$ raclopride administration. The basal level of dopamine increases after raclopride addition and the inset $\mathrm{CV}$, taken after stimulated dopamine has cleared, is consistent with dopamine. B) Raclopride significantly decreased evoked dopamine release (*, $\mathrm{p}<0.05, \mathrm{n}=7)$. C) Non-background subtracted current vs. time profile after the addition of raclopride showing the increase in baseline current. The inset CV is from the basal increase and is not stimulated release. The inset concentration vs. time profile shows a small, surge of spontaneous dopamine release. D) Non-background subtracted current vs. time profile from a rat brain slice after the addition of raclopride shows basline current increase similar to that found in Drosophila. The inset $\mathrm{CV}$ verifies the increase is due to dopamine. E.) Comparison of surges of basal dopamine after raclopride in flies with reduced DD2R expression and control flies. Dopamine events last longer and are of greater magnitude in controls. The $\mathrm{CV}$ for basal change in flies with underexpressing DDR2 has an additional peak at $-0.1 \mathrm{~V}$ likely due to a $\mathrm{pH}$ shift. F) Average number of dopamine surge events in 20 min after raclopride addition. Flies with reduced levels of DD2Rs have significantly fewer dopamine surges compared to control flies (Pearson chi squared test, $* \mathrm{p} \leq 0.05, \mathrm{n}=7$ ). 
blue-light stimulation to $30 \mathrm{~min}$ after raclopride addition. The CV inset in Figure $3.4 \mathrm{C}$ was taken 2 minutes post drug addition, and has a clear dopamine oxidation and reduction peak. This $\mathrm{CV}$ is not stimulated release but due to changes in basal dopamine. Long-term changes in basal levels are not typically studied with FSCV because the initial basal concentration cannot be determined and carbon-fiber microelectrodes are not stable over long periods of time (52). However, the change in basal current can be used to estimate the magnitude of a dopamine basal change. On average the baseline increases by about $4.3 \pm 1 \mathrm{nA}$, which would correspond to $370 \pm 100 \mathrm{nM}$ if the changes were due to only dopamine release. This large increase in basal dopamine might deplete internal stores leading to the lower levels of stimulated release. The increases in basal levels also confirm the action of the D2 receptor as an autoreceptor, as autoreceptors would be expected to regulate both basal and stimulated release. Although raclopride does not cause an increase in stimulated dopamine release, it does mediate dopaminergic signaling because basal increases are observed.

To investigate whether this large basal release of dopamine was an effect specific to Drosophila, similar experiments were performed in rat brain slices. Figure 3.4 D shows a non-background subtracted current versus time profile for the addition of raclopride to a rat brain slice of the caudate-putamen. A similar increase in baseline current was observed after raclopride and the CV is consistent with dopamine (inset). Therefore, raclopride can cause large increases in dopamine basal levels in mammals as well as Drosophila. The similarity between the response in Drosophila and mammals 
suggests that while the D2 receptor isoforms of the two species may be different, basic biology is conserved.

In addition to an overall increase in baseline, small surges of dopamine were observed in Drosophila after $5 \mu \mathrm{M}$ raclopride addition that were not elicited by the stimulation. The inset of Figure $3.4 \mathrm{C}$ shows an approximately $80 \mathrm{~s}$ surge with a dopamine concentration of $60 \mathrm{nM}$. Because these small changes occurred simultaneously with the large changes in baseline dopamine, the signal does not return to baseline. Figure 3.4 E displays another example dopamine surge after raclopride administration that was measured in a $t h-G A L 4$ wild-type fly that did not express $\mathrm{ChR} 2$ and was not stimulated. This surge is about $150 \mathrm{nM}$ and lasts $80 \mathrm{~s}$. The duration of dopamine surges ranged from 20 to $80 \mathrm{~s}$ and the concentration ranged from $50 \mathrm{nM}$ to $200 \mathrm{nM}$. The length of these dopamine surges is much longer than transient increases in dopamine detected in rats during behavioral experiments (ranging from 0.5 to 2 seconds) $(53,54)$ or after D2R antagonist addition ( 1 to $5 \mathrm{~s}$ ) (55); thus they may not be due to burst firing but to changes in basal firing. Raclopride has two effects on dopamine release in Drosophila, increasing overall basal levels and causing transient surges in dopamine release.

In order to demonstrate that the increase in dopamine after raclopride was due to DD2R, we investigated the effect of raclopride on dopamine release in flies with reduced levels of DD2R (th-GAL4;UAS-ds-DD2R). Large increases in baseline dopamine were not observed after raclopride; however, low concentration surges of dopamine were occasionally still observed (Figure 3.4 E). The CV from the fly with reduced DDR2 expression has a peak consistent with dopamine but also has an additional peak at $-0.1 \mathrm{~V}$, 
which has previously been associated with an acidic $\mathrm{pH}$ shift (56). The concentration of dopamine surges in control flies (th-GAL4) was on average $30 \%$ greater in control flies than in flies with reduced DDR2 expression (Figure 3.4 E). Dopamine events were also shorter in flies underexpressing DDR2, lasting only 5 to $15 \mathrm{~s}$ instead of 20 to $80 \mathrm{~s}$. Figure 3.4 F shows that the number of dopamine surges was significantly larger for control flies compared to those underexpressing DD2Rs $(n=7, p=0.05)$. These experiments demonstrate that flies underexpressing DDR2 do not have overall increases in basal dopamine levels and have fewer dopamine surges than control flies. Thus, DDR2 receptors are required for raclopride to affect dopamine levels. These experiments in the genetic mutant fly help distinguish that the effects of raclopride are mediated by DDR2 and not another autoreceptors, such as D3 receptors. Combining pharmacology and genetic mutant experiments make Drosophila a powerful model organism for neuroscience studies.

\subsection{5: Interactions of D2 receptors and DAT}

In mammals, an interaction between D2 receptors and the dopamine transporter (DAT) has been postulated because D2 agonists have been found to increase the kinetics of DAT, speeding up clearance (57). The extent of this effect is debated and varies by brain region and electrochemical detection method. Meiergerd et al. found a large effect of agonists on clearance in the striatum using rotating disk voltammetry, while Joseph et al. and Mathews et al. found no effect using FSCV in the striatum and nucleus accumbens, respectively $(34,58,59)$. Our study provides no evidence that D2 agonists 
increase DAT kinetics in Drosophila. For the agonists, bromocriptine and quinpirole non-significantly increase $t_{50}$, the opposite of the expected effect. For the antagonists, only flupenthixol slowed clearance while butaclamol and haloperidol did not, suggesting this is not a widespread effect of antagonists. An interaction between the D2 receptor and DAT may not occur or the effect may not be measured with FSCV.

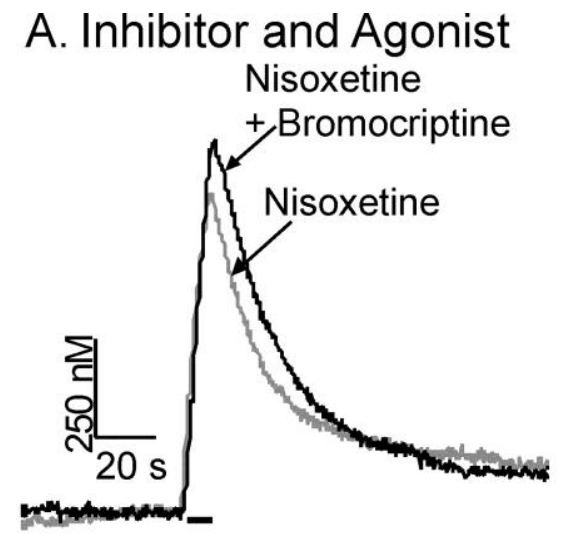

C. Inhibitor and Antagonist

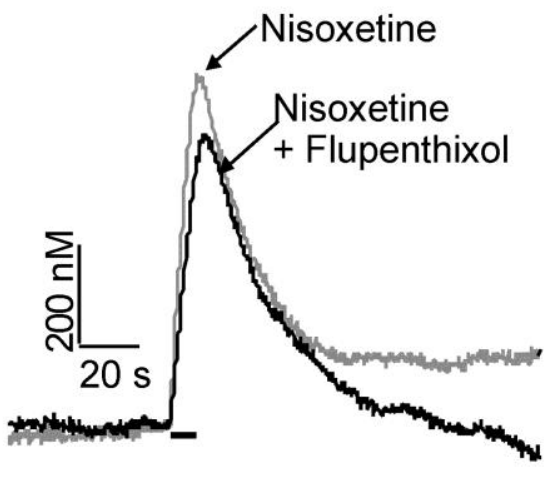

B. Nisoxetine + Bromocriptine
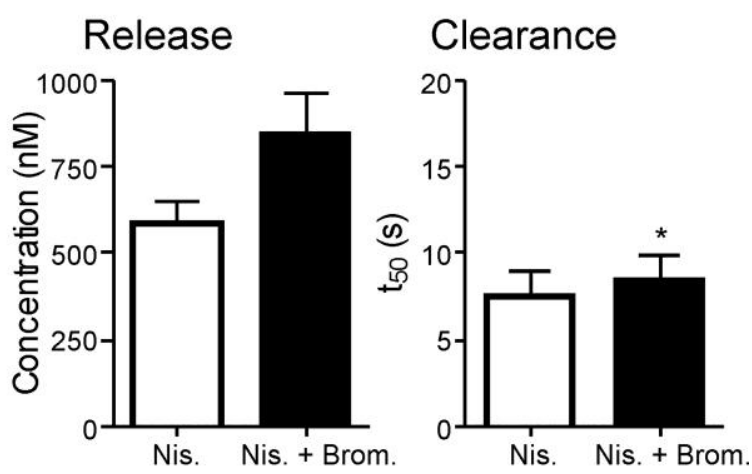

D. Nisoxetine + Flupenthixol

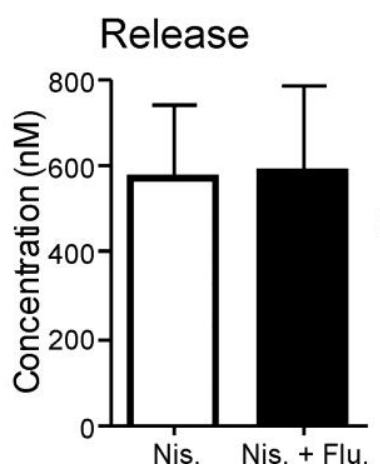

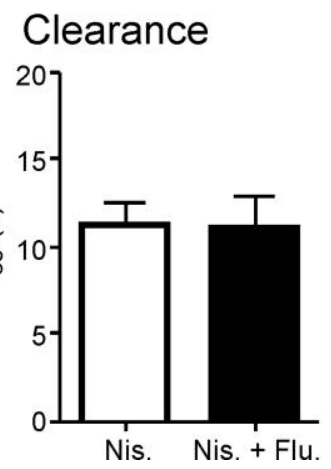

Figure 3.5: Effects of the DAT inhibitor with D2R agonists and antagonists. (A) Concentrationtime profile for a VNC incubated in $50 \mu \mathrm{M}$ nisoxetine followed by addition of $50 \mu \mathrm{M}$ bromocriptine. (B) Clearance of dopamine is significantly increased in the presence of both nisoxetine and bromocriptine $(n=6)$. The effect of $50 \mu \mathrm{M}$ bromocriptine is suppressed in the presence of $50 \mu \mathrm{M}$ nisoxetine $(n=6)$. (C) Concentration-time profile for a VNC incubated in 5 $\mu \mathrm{M}$ nisoxetine followed by addition of $5 \mu \mathrm{M}$ flupenthixol. (D) Clearance is not significantly changed in VNCs incubated in nisoxetine followed by flupenthixol. The effect of flupenthixol is suppressed in the presence of nisoxetine. All statistics are from paired $t$ tests of release or clearance before and after addition of drug. ${ }^{*} p<0.05 .{ }^{*} p<0.01 .{ }^{* * *} p<0.001$. 
To examine this possible interaction further, nisoxetine, an inhibitor of the Drosophila dopamine transporter (dDAT), was administered before a D2 agonist or antagonist (Figure 3.5 ). The $\mathrm{t}_{50}$ increases significantly after nisoxetine and bromocriptine, similar to data for bromocriptine alone, and opposite of the effect expected if the D2 agonist had a facilitatory effect on DAT. Therefore, these data do not reveal any cooperative effect of an agonist on DAT activity. After nisoxetine and bromocriptine there is a non-significant $(\mathrm{p}=0.0523)$ trend towards increased release compared to nisoxetine alone (Figure 3.5 A and B). Uptake inhibition causes the D2 agonist to have the opposite effect it had when administered alone. Increased basal dopamine levels after nisoxetine may saturate D2 receptors, and thus there is no decrease in release after D2 agonist administration.

When the same experiments were repeated with a D2 antagonist, there was no difference in either release or clearance after the addition of flupenthixol compared to nisoxetine alone. (Figure 3.5 C and D). Thus, there does not appear to be any facilitatory interaction of D2 receptors for uptake that is blocked by the antagonist. However, administration of an uptake inhibitor does inhibit the effect of a D2 antagonist on release. In mammals, D2R administration after DAT inhibition did not increase high-frequency stimulated release in rats (60) and DAT inhibitors and D2 antagonists increase basal levels of dopamine, which may lead to less DA available for stimulated release (55). Similarly, our results show that nisoxetine blocked the ability of the antagonist to increase dopamine release in Drosophila. While DAT inhibition affected the regulation of stimulated release by autoreceptors, a direct interaction of D2 receptors and DAT for 
clearance was not identified. Future experiments could further probe the complex regulation of release governed by uptake and autoreceptors in Drosophila. 


\section{3: Conclusion}

We have chemically investigated the effect of the Drosophila dopamine-2 receptor on regulating dopamine release. The decrease in stimulated dopamine release in the presence of dopamine agonists and the increase in release in the presence of antagonists is consistent with the DDR2 acting as an autoreceptor. These studies were modeled after mammalian studies, which probed autoreceptor functionality by electrically stimulating dopaminergic fibers and detecting dopamine with fast-scan cyclic voltammetry $(28,34,60,61)$. This specific stimulation protocol and the fast nature of the detection led to a probing of presynaptic effects (31). Similarly, in our study, optical stimulation of ChR2 located specifically in dopaminergic terminals would also investigate primarily presynaptic regulation. Thus, the pharmacological effects are unlikely to be due to downstream effects caused by activation of postsynaptic dopamine receptors. The effects of D2 agonists and antagonists on stimulated dopamine release in Drosophila are analogous to results in mammals; this supports the conclusion that the $\mathrm{DD} 2 \mathrm{R}$ is functioning as an autoreceptor, regulating the release of dopamine. While no interaction facilitating uptake was observed for D2 receptors and DAT, disruption of dopamine signaling with an uptake inhibitor did alter the effects of D2 drugs on dopamine release. Because autoreceptors play such an important role in human disease etiology, the conservation of autoreceptors between species makes Drosophila a useful model for studying dopaminergic diseases. 


\section{4: Experimental Methods}

\subsection{1: Chemicals}

Unless specified, all chemicals were from Sigma-Aldrich (St. Loius, MO). All solutions were made using MilliQ water (Millipore, Billerica, MA). All calibrations and dissections were made in a modified Schneider's buffer $\left(15.2 \mathrm{mM} \mathrm{MgSO}_{4}, 21 \mathrm{mM} \mathrm{KCl}\right.$, $3.3 \mathrm{KH}_{2} \mathrm{PO}_{4}, 36 \mathrm{mM} \mathrm{NaCl}, 5.8 \mathrm{mM} \mathrm{NaH}_{2} \mathrm{PO}_{4}, 5.4 \mathrm{mM} \mathrm{CaCl}_{2}, 11.1 \mathrm{mM}$ glucose, $5.3 \mathrm{mM}$ trehalose, $\mathrm{pH}$ 6.2). $10 \mathrm{mM}$ stock solutions of all agonists and antagonists were made in DMSO and were diluted with modified Schneider's buffer. The final concentration in the bath around the Drosophila CNS was $50 \mu \mathrm{M}$ bromocriptine, $50 \mu \mathrm{M}$ quinpirole, $5 \mu \mathrm{M}$ flupenthixol, $5 \mu \mathrm{M}$ butaclamol, $5 \mu \mathrm{M}$ haloperidol, or $5 \mu \mathrm{M}$ raclopride. For nisoxetine experiments, the final concentration in the bath was $50 \mu \mathrm{M}$ nisoxetine for agonist and 5 $\mu \mathrm{M}$ nisoxetine for antagonist experiments. A $10 \mathrm{mM}$ stock solution of dopamine for electrode calibration was made in $0.1 \mathrm{M}$ perchloric acid and diluted to $1 \mu \mathrm{M}$ with modified Schneider's buffer. Larvae were fed $10 \mathrm{mM}$ all trans-retinal mixed with Red Star Yeast (Milwaukee, WI) and water.

\subsection{2: Electrochemical Measurements}

Carbon-fiber microelectrodes were made by aspirating single T-650 carbon fibers (Cytec Engineering Materials, West Patterson, NJ) into $1.2 \mathrm{~mm}$ X $0.68 \mathrm{~mm}$ glass capillaries (A-M Systems, Carlsburg, WA). After pulling in a vertical pipette puller 
(Narishige PE-21, East Meadow, NY), electrodes were trimmed to 40-60 $\mu \mathrm{m}$. Electrodes were then dipped for $30 \mathrm{~s}$ into heated $\left(85^{\circ} \mathrm{C}\right.$ ) Epon Resin 828 (Miller-Stephenson, Danbury, CT) mixed with 14\% (w/w) m-phenylenediamine hardener (Fluka, Milwaukee, WI). After curing in an oven at $100^{\circ} \mathrm{C}$ for $2 \mathrm{~h}$ followed by $150{ }^{\circ} \mathrm{C}$ overnight, the electrodes were soaked in isopropanol and backfilled with $1 \mathrm{M} \mathrm{KCl}$ before use.

Tar Heel CV software (gift of Mark Wightman, University of North Carolina) was used to collect and analyze data from a Dagan Chem-Clamp potentiostat (Dagan, Minneapolis, MN). A triangular waveform was generated and data digitized by a homemade breakout box with PCI 6052 and 6711 boards (National Instruments, Austin, TX). Every $100 \mathrm{~ms}$ the electrode was scanned from -0.4 to $1.3 \mathrm{~V}$ and back at a scan rate of $400 \mathrm{~V} / \mathrm{s} . \mathrm{An} \mathrm{Ag} / \mathrm{AgCl}$ reference electrode was placed in the Petri dish near the ventral nerve cord.

Electrodes were allowed to cycle for 15 minutes prior to implantation. Peak oxidation current from the collected cyclic voltammograms was converted into concentration using a postelectrode calibration with $1 \mu \mathrm{M}$ dopamine. For all drug experiments, a second calibration was performed in the presence of drug to account for possible drug effects on the electrode sensitivity.

\subsection{3: Preparation of Ventral Nerve Cords}

Flies containing UAS-ChR2 were crossed to flies expressing th-GAL4 (a gift from Jay Hirsh, University of Virginia) to generate homozygous lines with a th-GAL4;UASChR2 genotype. Flies expressing th-GAL4 were crossed with flies containing UAS-ds- 
DD2R (a gift from Jay Hirsh, University of Virginia) to generate heterozygous lines with a th-GAL4;UAS-ds-DD2R genotype. Three-day-old, wandering $3^{\text {rd }}$ instar $t h$-GAL4;UASChR2 larvae were selected based on size and activity level, fed trans-retinal for 2 days and kept in the dark. Five-day-old larvae were selected based on size, the CNS was dissected out in modified Schneider's buffer and the optic lobes removed as previously described. $(10,11)$ Once isolated, the ventral nerve cord (VNC) was adhered to the bottom of a Petri dish (Becton Dickinson, Franklin Lakes, NJ) with $3 \mathrm{~mL}$ of buffer. Using a 40x water immersion lens, an electrode was inserted into the neuropil, a region dense with dopamine cell bodies and terminals. For control experiments, an initial $5 \mathrm{~s}$ blue light stimulation was used to evoke dopamine release followed by the addition of $1 \mathrm{~mL}$ buffer and $5 \mathrm{~s}$ stimulations were repeated at $15 \mathrm{~min}$ intervals. For drug experiments, an initial $5 \mathrm{~s}$ blue light stimulation was performed, then $1 \mathrm{~mL}$ of a solution containing the drug was added to the buffer around the nerve cord, and $5 \mathrm{~s} \mathrm{stimulations} \mathrm{repeated} \mathrm{at} 15$ min intervals.(10) For pre-incubated experiments, dissected nerve cords were incubated with drug in the dark for 15-20 min prior to blue-light stimulation.

\subsection{4: Statistical Analysis}

Data were analyzed using GraphPad Prism (GraphPad Software, San Diego, CA). Paired t-tests were used to compare release before and after drugs with the exception of pre-incubation with drug, where unpaired t-tests were used. Data were considered different at a 95\% confidence level. Error bars are standard error of the mean. Spontaneous changes in baseline dopamine were identified using CV matching software 
in TarHeel CV. A Pearson's chi squared test was used to determine if the number of dopamine release events after raclopride was significantly different in flies with reduced levels of dopamine compared to controls (SPSS, Somers, NY). Dopamine event CVs were compared to a dopamine template voltammogram generated from stimulated release in a larval ventral nerve cord expressing Channelrhodopsin2 in dopaminergic neurons.(52) A template of stimulated release CV could not be obtained in the flies with reduced DD2R expression or control flies because they lack Channelrhodopsin2. A correlation coefficient of 0.70 was used as the criteria for the change being identified as dopamine. Because the template voltammogram was obtained from a different animal, the correlation coefficients were not as high as for experiments where stimulated release is compared to spontaneous release in the same animal.(55)

\subsection{5: Rat brain slice experiment}

Adult, male Sprague-Dawley rats (250-350 g) purchased from Charles River were housed in a vivarium and given food and water ab libitum. All experiments were approved by the Animal Care and Use Committee of the University of Virginia, and were performed by Megan Pajaski.

Rat brain slice experiments were performed as previously described.(62) Briefly, rats were anesthetized with isoflurane and then beheaded. The brain was removed and placed for 2-3 minutes ice-cold artificial cerebral spinal fluid (aCSF) saturated with 95\% $\mathrm{O}_{2}: 5 \% \mathrm{CO}_{2}$ (aCSF: $126 \mathrm{mM} \mathrm{NaCl}, 2.5 \mathrm{mM} \mathrm{KCl}, 1.2 \mathrm{mM} \mathrm{NaH} \mathrm{PO}_{4}, 2.4 \mathrm{mM} \mathrm{CaCl} 2 \cdot 2 \mathrm{H}_{2} \mathrm{O}$ , $1.2 \mathrm{mM} \mathrm{MgCl} 26 \mathrm{H}_{2} \mathrm{O}, 25 \mathrm{mM} \mathrm{NaHCO} 3,11 \mathrm{mM}$ glucose and $15 \mathrm{mM}$ 
tris(hydroxymethyl) aminomethane, with the $\mathrm{pH}$ adjusted to 7.4). $400 \mu \mathrm{m}$ slices through the caudate-putamen were made using a vibratome (Leica VT1000S, Bannockburn, IL) and allowed to recover in aCSF for at least an hour before the experiment.

In the brain slice chamber, room temperature aCSF was pumped over the slice at a rate of $1 \mathrm{~mL} / \mathrm{min}$ with a multi-channel, manual-control pump (Watson-Marlo 205U, Wilmington, MA). The carbon-fiber microelectrode was inserted so that the tip was approximately $75 \mu \mathrm{m}$ below the top of the slice and the waveform was applied for 30 minutes before data was collected. The electrode position corresponded to approximately the following coordinates from bregma: $+1.2 \mathrm{~mm}$ anterior-posterior, +2.1 to $+2.3 \mathrm{~mm}$ medial-lateral and -4.2 to $-4.5 \mathrm{~mm}$ dorsal-ventral. The bipolar stimulating electrode (Plastics One, Inc., Roanoke, VA) was placed about $500 \mu \mathrm{m}$ from the working electrode, so that the prongs of the stimulating electrode and the working electrode formed a triangle. The slice was stimulated with one $300 \mu \mathrm{A}$ biphasic pulse, $4 \mathrm{~ms}$ long, using a BSI-950 Biphasic Stimulus Isolator (Dagan). 


\subsection{References}

1. Neve, K. A. (2004) Dopamine Receptors. In Encyclopedia of Biological Chemistry, pp 817-822, Elsevier, New York.

2. Creese I, Burt DR, and Snyder SH. (1976) Dopamine receptor binding predicts clinical and pharmacological potencies of antischizophrenic drugs, Science 192, 481-3.

3. Abi-Dargham A, Rodenhiser J, Printz D, Zea-Ponce Y, Gil R, Kegeles LS, Weiss R, Cooper TB, Mann JJ, Van Heertum RL, Gorman JM, and Laruelle M. (2000) Increased baseline occupancy of D2 receptors by dopamine in schizophrenia, $\mathrm{P}$. Natl. Acad. Sci. USA 97, 8104-9.

4. Calabresi P, Saiardi A, Pisani A, Baik JH, Centonze D, Mercuri NB, Bernardi G, and Borrelli E. (1997) Abnormal synaptic plasticity in the striatum of mice lacking dopamine D2 receptors, J. Neurosci. 17, 4536-44.

5. Gurevich, E. V., and Gurevich, V. V. (2010) Dopamine Receptors and the Treatment of Parkinson's Disease. In The Dopamine Receptors, pp 525-584, Humana Press.

6. Eilam, D., and Szechtman, H. (1989) Biphasic effect of D-2 agonist quinpirole on locomotion and movements, Eur. J. Pharmacol. 161, 151-157.

7. Jackson DM, Johansson C, Lindgren LM, and Bengtsson A. (1994) Dopamine receptor antagonists block amphetamine and phencyclidine-induced motor stimulation in rats, Pharmacol. Biochem. Be. 48, 465-71.

8. Porzgen, P., Park, S. K., Hirsh, J., Sonders, M. S., and Amara, S. G. (2001) The Antidepressant-Sensitive Dopamine Transporter inDrosophila melanogaster: A Primordial Carrier for Catecholamines, Mol. Pharmacol. 59, 83-95.

9. Neckameyer, W., Holt, B., and Paradowski, T. (2005) Biochemical Conservation of Recombinant Drosophila Tyrosine Hydroxylase with its Mammalian Cognates, Biochem. Genet. 43, 425-443.

10. Vickrey, T. L., Condron, B., and Venton, B. J. (2009) Detection of Endogenous Dopamine Changes in Drosophila melanogaster Using Fast-Scan Cyclic Voltammetry, Anal. Chem. 81, 9306-9313. 
11. Borue, X., Cooper, S., Hirsh, J., Condron, B., and Venton, B. J. (2009)

Quantitative evaluation of serotonin release and clearance in Drosophila, $\mathrm{J}$.

Neurosci. Methods 179, 300-308.

12. Caron MG, and Lefkowitz RJ. (1993) Catecholamine receptors: structure, function, and regulation, Recent Prog. Horm. Res. 48, 277-90.

13. Liu, I. S. C., George, S. R., and Seeman, P. (2000) The human dopamine D2Longer receptor has a high-affinity state and inhibits adenylyl cyclase, Mol. Brain Res. 77, 281-284.

14. Lindgren, N. (2003) Distinct Roles of Dopamine D2L and D2S Receptor Isoforms in the Regulation of Protein Phosphorylation at Presynaptic and Postsynaptic Sites, P. Natl. Acad. Sci. USA 100, 4305.

15. Tan, S. (2003) Dopaminergic mouse mutants: investigating the roles of the different dopamine receptor subtypes and the dopamine transporter, Int. Rev. Neurobiol. 54, 145.

16. Mayfield, R. D., and Zahniser, N. R. (2001) Dopamine D2 Receptor Regulation of the Dopamine Transporter Expressed in Xenopus laevis Oocytes Is VoltageIndependent, Mol. Pharmacol. 59, 113-121.

17. Guennoun, R., and Bloch, B. (1991) D2 dopamine receptor gene expression in the rat striatum during ontogeny: an in situ hybridization study, Dev. Brain Res. 60, 79-87.

18. Liu, Y. F. (1992) Differential Sensitivity of the Short and Long Human Dopamine D2 Receptor Subtypes to Protein Kinase C, J. Neurochem. 59, 2311-2317.

19. Suo, S., Sasagawa, N., and Ishiura, S. (2003) Cloning and characterization of a Caenorhabditis elegans D2-like dopamine receptor, J. Neurochem. 86, 869-878.

20. Hearn MG, Ren Y, McBride EW, Reveillaud I, Beinborn M, and Kopin AS. (2002) A Drosophila dopamine 2-like receptor: Molecular characterization and identification of multiple alternatively spliced variants, P. Natl. Acad. Sci. USA 99, 14554-9.

21. Draper I, Kurshan PT, McBride E, Jackson FR, and Kopin AS. (2007) Locomotor activity is regulated by D2-like receptors in Drosophila: an anatomic and functional analysis, Dev. Neurobio. 67, 378-93.

22. Yellman, C., Tao, H., He, B., and Hirsh, J. (1997) Conserved and sexually dimorphic behavioral responses to biogenic amines in decapitated Drosophila, P. Natl. Acad. Sci. USA 94, 4131-4136. 
23. Hirsh, J. (1997) Decapitated Drosophila: A Novel System for the Study of Biogenic Amines, Adv. Pharmacol. 42, 945-948.

24. Vickrey, T. L., and Venton, B. J. (2011) Drosophila Dopamine2-like Receptors Function as Autoreceptors, ACS Chem. Neurosci. 2, 723-729.

25. Robinson, D. L., Venton, B. J., Heien, M. L. A. ., and Wightman, R. M. (2003) Detecting Subsecond Dopamine Release with Fast-Scan Cyclic Voltammetry in Vivo, Clin Chem 49, 1763-1773.

26. Heien, M. L. A. V., Khan, A. S., Ariansen, J. L., Cheer, J. F., Phillips, P. E. M., Wassum, K. M., and Wightman, R. M. (2005) Real-time measurement of dopamine fluctuations after cocaine in the brain of behaving rats, P. Natl. Acad. Sci. USA 102, 10023-10028.

27. Finnegan, J. M., Pihel, K., Cahill, P. S., Huang, L., Zerby, S. E., Ewing, A. G., Kennedy, R. T., and Wightman, R. M. (1996) Vesicular Quantal Size Measured by Amperometry at Chromaffin, Mast, Pheochromocytoma, and Pancreatic $\beta$ Cells, J. Neurochem. 66, 1914-1923.

28. Kennedy, R. T., Jones, S. R., and Wightman, R. M. (1992) Dynamic Observation of Dopamine Autoreceptor Effects in Rat Striatal Slices, J. Neurochem. 59, 449455.

29. Bull, D. R., Bakhtiar, R., and Sheehan, M. J. (1991) Characterization of dopamine autoreceptors in the amygdala: A fast cyclic voltammetric study in vitro, Neurosci. Lett. 134, 41-44.

30. Stamford JA, Kruk ZL, and Millar J. (1988) Actions of dopamine antagonists on stimulated striatal and limbic dopamine release: an in vivo voltammetric study, Brit. J. Pharmacol. 94, 924-32.

31. Benoit-Marand, M., Borrelli, E., and Gonon, F. (2001) Inhibition of Dopamine Release Via Presynaptic D2 Receptors: Time Course and Functional Characteristics In Vivo, J. Neurosci. 21, $9134-9141$.

32. Tissari, A. H. (1983) Autoreceptors mediate the inhibition of dopamine synthesis by bromocriptine and lisuride in rats, Eur. J. Pharmacol. 91, 463-8.

33. Al-Fulaij, M. A., Ren, Y., Beinbom, M., and Kopin, A. S. (2007) NEUROPHARMACOLOGY - Identification of Amino Acid Determinants of Dopamine 2 Receptor Synthetic Agonist Function, J. Pharmacol. Exp. Ther. 321, 298. 
34. Maina, F. K., and Mathews, T. A. (2010) Functional Fast Scan Cyclic Voltammetry Assay to Characterize Dopamine D2 and D3 Autoreceptors in the Mouse Striatum, ACS Chem. Neuro. 1, 450-462.

35. Bogomolova, E. V., Rauschenbach, I. Y., Adonyeva, N. V., Alekseev, A. A., Faddeeva, N. V., and Gruntenko, N. E. (2010) Dopamine down-regulates activity of alkaline phosphatase in Drosophila: The role of D2-like receptors, J. Insect. Physiol. 56, 1155-1159.

36. Usiello, A., Baik, J.-H., Rouge-Pont, F., Picetti, R., Dierich, A., LeMeur, M., Piazza, P. V., and Borrelli, E. (2000) Distinct functions of the two isoforms of dopamine D2 receptors, Nature 408, 199-203.

37. Pierce, R. C., Duffy, P., and Kalivas, P. W. (1995) Sensitization to cocaine and dopamine autoreceptor subsensitivity in the nucleus accumbens, Synapse 20, 3336.

38. Hilditch A, and Drew GM. (1981) Characteristics of the dopamine receptors in the rabbit isolate splenic artery, Eur. J. Pharmacol. 72, 287-96.

39. Goldberg, L. (1979) Peripheral pre- and post-synaptic dopamine receptors: are they different from dopamine receptors in the central nervous system?, Commun. Psychopharmac. 3, 447-56.

40. Gattaz, W. F., Diehl, A., Geuppert, M. S., Hubrich, P., Schmitt, A., Linde, I., Maras, A., and Dittmann, R. W. (2004) Olanzapine versus Flupenthixol in the Treatment of Inpatients with Schizophrenia: A Randomized Double-Blind Trial, Pharmacopsychiatry 37, 279-285.

41. Hyttel, J. (1977) Changes in dopamine synthesis rate in the supersensitivity phase after treatment with a single dose of neuroleptics, Psychopharmacology 51, 205207.

42. Eshleman, A. J., Neve, R. L., Janowsky, A., and Neve, K. A. (1995) Characterization of a recombinant human dopamine transporter in multiple cell lines., J. Pharmacol. Exp. Ther. 274, 276 -283.

43. Clark, M. L., Paredes, A., Costiloe, J. P., and Wood, F. (1977) Evaluation of butaclamol in chronic schizophrenic patients, J. Clin. Pharmacol. 17, 529-536.

44. Onali, P., Olianas, M. C., and Gessa, G. L. (1985) Characterization of dopamine receptors mediating inhibition of adenylate cyclase activity in rat striatum, Mol. Pharmacol. 28, 138-145. 
45. Hartman, D. S., and Civelli, O. (1996) Molecular Attributes of Dopamine Receptors: New Potential for Antipsychotic Drug Development, Ann. Med. 28, 211-219.

46. Wu, Q., Reith, M. E. A., Walker, Q. D., Kuhn, C. M., Carroll, F. I., and Garris, P. A. (2002) Concurrent Autoreceptor-Mediated Control of Dopamine Release and Uptake during Neurotransmission: An In Vivo Voltammetric Study, J. Neurosci. 22, 6272-6281.

47. Garris, P. A., Budygin, E. A., Phillips, P. E. M., Venton, B. J., Robinson, D. L., Bergstrom, B. P., Rebec, G. V., and Wightman, R. M. (2003) A role for presynaptic mechanisms in the actions of nomifensine and haloperidol, Neuroscience 118, 819-829.

48. Braun, A. (1997) Interactions between D1 and D2 dopamine receptor family agonists and antagonists: the effects of chronic exposure on behavior and receptor binding in rats and their clinical implications., J. Neural Transm. 104, 341.

49. Hess, E., Norman, A., and Creese, I. (1988) Chronic treatment with dopamine receptor antagonists: behavioral and pharmacologic effects on D1 and D2 dopamine receptors, J. Neurosci. 8, 2361 -2370.

50. Marcus, M. M., Nomikos, G. G., and Svensson, T. H. (1996) Differential actions of typical and atypical antipsychotic drugs on dopamine release in the core and shell of the nucleus accumbens, Eur. Neuropsychopharm. 6, 29-38.

51. Hillegaart, V., and Ahlenius, S. (1987) Effects of Raclopride on Exploratory Locomotor Activity, Treadmill Locomotion, Conditioned Avoidance Behaviour and Catalepsy in Rats: Behavioural Profile Comparisons between Raclopride, Haloperidol and Preclamol, Pharmacol. Toxicol. 60, 350-354.

52. Heien, M. L. A. V., Khan, A. S., Ariansen, J. L., Cheer, J. F., Phillips, P. E. M., Wassum, K. M., Wightman, R. M., and Murray, R. W. (2005) Real-Time Measurement of Dopamine Fluctuations after Cocaine in the Brain of Behaving Rats, P. Natl. Acad. Sci. USA 102, 10023-10028.

53. Robinson, D. L., Phillips, P. E. M., Budygin, E. A., Trafton, B. J., Garris, P. A., and Wightman, R. M. (2001) Sub-second changes in accumbal dopamine during sexual behavior in male rats, Neuroreport 12, 2549-2552.

54. Robinson, D. L., Heien, M. L. A. V., and Wightman, R. M. (2002) Frequency of Dopamine Concentration Transients Increases in Dorsal and Ventral Striatum of Male Rats during Introduction of Conspecifics, J. Neurosci. 22, 10477 -10486. 
55. Venton, B. J., and Wightman, R. M. (2007) Pharmacologically induced, subsecond dopamine transients in the caudate-putamen of the anesthetized rat, Synapse 61, 37-39.

56. Takmakov, P., Zachek, M. K., Keithley, R. B., Bucher, E. S., McCarty, G. S., and Wightman, R. M. (2010) Characterization of Local pH Changes in Brain Using Fast-Scan Cyclic Voltammetry with Carbon Microelectrodes, Anal. Chem. 82, 9892-9900.

57. Bolan, E. A., Kivell, B., Jaligam, V., Oz, M., Jayanthi, L. D., Han, Y., Sen, N., Urizar, E., Gomes, I., Devi, L. A., Ramamoorthy, S., Javitch, J. A., Zapata, A., and Shippenberg, T. S. (2007) D2 Receptors Regulate Dopamine Transporter Function via an Extracellular Signal-Regulated Kinases 1 and 2-Dependent and Phosphoinositide 3 Kinase-Independent Mechanism, Mol. Pharmacol. 71, 1222 1232.

58. Meiergerd, S. M., Patterson, T. A., and Schenk, J. O. (1993) D2 Receptors May Modulate the Function of the Striatal Transporter for Dopamine: Kinetic Evidence from Studies In Vitro and In Vivo, J. Neurochem. 61, 764-767.

59. Joseph, J. D., Wang, Y.-M., Miles, P. R., Budygin, E. A., Picetti, R., Gainetdinov, R. R., Caron, M. G., and Wightman, R. M. (2002) Dopamine autoreceptor regulation of release and uptake in mouse brain slices in the absence of D3 receptors, Neuroscience 112, 39-49.

60. Wu, Q., Reith, M. E. A., Walker, Q. D., Kuhn, C. M., Carroll, F. I., and Garris, P. A. (2002) Concurrent Autoreceptor-Mediated Control of Dopamine Release and Uptake during Neurotransmission: An In Vivo Voltammetric Study, J. Neurosci. $22,6272-6281$.

61. Schmitz, Y., Schmauss, C., and Sulzer, D. (2002) Altered Dopamine Release and Uptake Kinetics in Mice Lacking D2 Receptors, The Journal of Neuroscience 22, $8002-8009$.

62. Pajski, M. L., and Venton, B. J. (2010) Adenosine Release Evoked by Short Electrical Stimulations in Striatal Brain Slices Is Primarily Activity Dependent, ACS Chem. Neuro. 1, 775-787. 


\section{Chapter 4}

\section{Voltammetric assessment of dopamine transporter activity in Drosophila}

"Education is the most powerful weapon which you can use to change the world."

Nelson Mandela 


\title{
Chapter 4: Voltammetric assessment of dopamine transporter activity in Drosophila
}

\author{
Abstract \\ Dopamine transporters regulate neurotransmission and are important in diseases \\ such as addiction and ADHD. The Drosophila dopamine transporter (dDAT) has \\ previously been shown to be highly analogous to the mammalian DAT; however, \\ characterization of dopamine transporter kinetics in intact, Drosophila larvae has not \\ been reported. In this study, we use a carbon-fiber microelectrode implanted into a larval \\ CNS to measure clearance kinetics of dopamine with fast-scan cyclic voltammetry. To \\ examine endogenous release, Channelrhodopsin 2 was expressed in dopamine neurons, \\ and different amounts of release were optically evoked to estimate the Michaelis-Menten \\ kinetics constants. However, with endogenous release it is not possible to evoke \\ concentrations close to saturation, so pressure ejection was used to apply exogenous \\ dopamine from a micropipette implanted $15-20 \mu \mathrm{m}$ away from the microelectrode. The \\ maximal rate of uptake, $V_{\max }$, was estimated to be $0.13 \pm 0.1 \mu \mathrm{M} / \mathrm{s}$ and $\mathrm{K}_{\mathrm{m}}$ was estimated \\ to be $1.7 \pm 0.8 \mu \mathrm{M}$. Clearance of exogenously-applied dopamine was significantly \\ reduced in fumin mutants, which lack a functional DAT, and kinetic constants in these \\ mutants were used to estimate and correct for diffusion of dopamine. Clearance was also \\ significantly reduced following treatment with $50 \mu \mathrm{M}$ cocaine, a DAT inhibitor. Flies \\ overexpressing DAT showed an increased rate of clearance. In addition, a line of \\ dopamine deficient flies had no significant changes in clearance. This method will be \\ useful for assessing the role of the dopamine transporter in regulating neurotransmission \\ and in understanding the underlying mechanisms of drug addiction.
}




\section{1: Introduction}

The neurotransmitter dopamine mediates critical functions such as locomotor activity, reward, cognition and motivation. Dopamine dysregulation is implicated in the pathophysiology of disorders such as attention deficit hyperactivity disorder (ADHD), schizophrenia and Parkinson's disease. The dopamine transporter (DAT) is a plasma membrane protein expressed in dopaminergic neurons. DATs act to regulate dopamine by rapidly transporting released dopamine from the extracellular space back into dopaminergic neurons, which is the main mechanism of synaptic dopamine clearance (1). Consequently, DAT is responsible for regulating the duration of dopamine signaling and the amount of dopamine available for signaling. DAT is also a target for drugs of abuse, such as cocaine and amphetamine, and drugs used to treat ADHD, which inhibit DAT activity and result in increased synaptic dopamine levels (2).

Real-time, in vivo and in situ measurements of dopamine release and dopamine clearance due to DAT activity have been studied in rodents using both pharmacological and genetic approaches (3-6). Psychostimulants such as cocaine and amphetamine inhibit DAT activity, prolong dopamine clearance and increase stereotypic behavior (4). Mice lacking functional dopamine transporters exhibit prolonged dopamine signaling, show limited responsiveness to DAT inhibitors, and are hyperlocomotive; mice overexpressing DAT show increased rates of uptake and increased locomotor response to amphetamine $(1,7,8)$. While mice with genetically altered DAT expression levels can provide a model system to study the role of DAT in dopamine homeostasis, mouse genetic models are 
more difficult and take more time (1-2 years versus 1-2 months) to produce compared to Drosophila genetic models.

Drosophila is an advantageous model organism because in comparison to mammalian models, Drosophila is easier to genetically modify, has larger numbers of progeny and shorter life spans. Genes are highly conserved between Drosophila and vertebrates including the basic components responsible for dopaminergic signaling (9). Genes encoding the Drosophila DAT (dDAT) share approximately 50\% sequence identity with mammalian DAT genes, and both transporters have similar protein motifs and substrate affinities for dopamine (9). Drosophila fumin mutants, which lack a functional dopamine transporter, show hyperactivity and disrupted sleep. These phenotypes are strikingly similar to those observed in DA knockout mice, and are likely caused by elevated dopamine persistence and release $(7,10-12)$. Development of a largescale screening method to assess DAT activity in Drosophila-DAT mutants would help elucidate the underlying homeostatic mechanisms that control dopaminergic signaling.

Few real-time measurements of dopamine release and clearance have been made in Drosophila due to the small size of its central nervous system (CNS), which is approximately $50 \mu \mathrm{m}$ x $150 \mu \mathrm{m}$. Our lab has recently developed a method to measure dopamine release and clearance in Drosophila using fast-scan cyclic voltammetry at carbon-fiber microelectrodes, which are approximately $7 \mu \mathrm{m}$ in diameter and amenable to the small size of the CNS. To evoke dopamine release, Channelrhodopsin-2 (ChR2), a blue-light activated cation channel, is expressed only in dopaminergic neurons and dopamine is released when blue-light is applied. Using ChR2-mediated dopamine release, 
we characterized dopamine signaling in the larval CNS $(13,14)$. However, we were unable to determine kinetic values such as $\mathrm{K}_{\mathrm{m}}$ and $\mathrm{V}_{\max }$ because evoked release did not result in dopamine concentrations high enough for saturation. In addition, to evaluate dopamine clearance in Drosophila DAT (dDAT) mutants using ChR2-mediated release, both the ChR2 mutation and DAT mutation would need to be co-expressed, which is difficult. To address these issues, a method that can measure clearance of larger concentrations, and can be used to quickly screen existing DAT mutants without additional genetic manipulation is needed.

Recently, the Ewing group used electrochemical techniques to measure clearance of exogenously applied dopamine in the protocerebral anterior medial region of the adult Drosophila brain $(12,15)$. They found that clearance of exogenously applied dopamine decreased following treatment of cocaine as well as decreased in fumin mutants. However, kinetic values such as $\mathrm{K}_{\mathrm{m}}$ and $\mathrm{V}_{\max }$ were not determined, and exogenous dopamine clearance was not compared to stimulated dopamine clearance. Development of a complementary method to use in larvae would allow dDAT to be studied throughout development, which could be important for understanding the relationship between DAT activity, dopamine homeostasis, and disease.

In this paper, we report two methods for the quantitative analysis of dopamine transporter activity in an intact, Drosophila larval CNS. We use fast-scan cyclic voltammetry at carbon-fiber microelectrodes to measure exogenous and endogenous dopamine clearance. We provide the first values for maximum velocity of uptake, $\mathrm{V}_{\max }$, and transporter affinity for dopamine, $\mathrm{K}_{\mathrm{m}}$, measured in intact, Drosophila tissue. We 
show that clearance is decreased in fumin mutants, and use these mutants to correct for dopamine diffusion. Clearance is also decreased in the presence of cocaine, and transporter affinity for dopamine in the presence of cocaine $\left(\mathrm{K}_{\mathrm{m}, \mathrm{obs}}\right)$ calculated. The method was used to test dDAT activity in a dopamine deficient mutant, $D T H g^{F S \ddagger}$; ple, which had normal clearance, indicating dopamine deficiency does not affect dDAT activity in Drosophila. Finally, a Drosophila mutant overexpressing dDAT, NP2785; $d D A T$, was tested and showed an overall increase in clearance. These studies demonstrate that this method can be used to screen Drosophila genetic mutants, and further validates Drosophila as a model system to study dopaminergic diseases. 


\subsection{Results and Discussion}

\subsection{1: Drosophila uptake via stimulated release}

Real-time measurement of DAT activity in Drosophila larvae has been limited by a lack of rapid detection techniques that are suitable for the small size of the Drosophila central nervous system (CNS). The CNS contains a dense network of different types of neurotransmitter terminals and synapses, called the neuropil. The lack of segmentation in the neuropil makes it difficult to initiate release from a single neuron type. Previously, we developed a method to initiate dopamine-specific release using Channelrhodopsin2 (ChR2) expressed in only dopaminergic neurons (13). In chapter 2 of this thesis, this method was used to demonstrate that release was neuronal, and to characterize dopamine release in the fly. Here, we use this same method to investigate Michaelis-Menten uptake parameters for DAT in an intact Drosophila tissue for the first time.

A carbon-fiber microelectrode was implanted into the CNS of a homozygous UAS-ChR2;th-GAL4 Drosophila larva and the duration of blue-light was varied. Figure 2.4 shows dopamine release for 3, 5, 7, 10, and $12 \mathrm{~s}$ of blue-light stimulation. Maximal dopamine concentration reaches a plateau after about $7 \mathrm{~s}$ of blue light exposure, and $7 \mathrm{~s}$ and $15 \mathrm{~s}$ stimulations are not significantly different $(\mathrm{n}=5$, paired $\mathrm{t}$-test, $\mathrm{p}=0.86)$. This plateau suggests that either maximal dopamine release has been achieved or that dopamine is cleared during the stimulation by dDAT, leading to a steady-state concentration. 
In order to estimate transporter affinity, $\mathrm{K}_{\mathrm{m}}$, and maximal rate of uptake, $\mathrm{V}_{\max }$, we must assume that all clearance is due to uptake. As described by Sabeti et al., we fit the decay portion of each peak with a single exponential decay function using a non-linear regression analysis:

$[\mathrm{DA}](t)=[\mathrm{DA}]_{\max } \times e^{-k t}$

Equation 4.1

where $[\mathrm{DA}]$ is the dopamine concentration at any given time, $t,[\mathrm{DA}]_{\max }$ is the maximum dopamine concentration and $\mathrm{k}$ is the first order rate constant (16). Figure 4.1A shows a current versus time plot with a $7 \mathrm{~s}$ blue-light stimulation (black line) that has been superimposed with a first-order exponential fit (yellow). Optimal fit $\left(r^{2} \geq 0.98\right)$ of the decay curve was achieved when the signal decay was fit from the time the stimulation ended until $80 \%$ of the signal decayed, similar to previous studies $(16,17)$. The firstorder rate constants were then used to calculate the initial velocity, $\mathrm{V}$, of dopamine clearance (Equation 4.2).

$V=k \bullet[\mathrm{DA}]_{\max }$

Equation 4.2

The initial velocity was plotted against the maximal dopamine concentration from multiple peaks obtained during variable length stimulations $(n=28$ from 8 different CNS's). A non-linear regression analysis was performed by fitting the collected data to the Michaelis-Menten equation:

$V=\frac{V \max _{\max } \bullet[\mathrm{DA}]_{\max }}{K_{m}+[\mathrm{DA}]_{\max }}$

Equation 4.3

However, the range of concentrations of dopamine released during blue-light stimulations was not large enough to accurately estimate $\mathrm{V}_{\max }$ or $\mathrm{K}_{\mathrm{m}}$ (Figure $4.1 \mathrm{C}$ ). In addition, in order to evoke dopamine release in Drosophila, UAS-ChR2;th-GAL4 must be 
homozygous. Drosophila has a total of 4 chromosomes: 3 autosomal and 1 gonosomal. The UAS-ChR2;th-GAL4 homozygote has two copies of ChR2 on the second chromosome and two copies of th on the third chromosome. Although genetic manipulations are easier in Drosophila in comparison to mammals, it is still difficult to co-express multiple mutations on the same chromosomes and have them be homozygous. A method that could measure real-time variations in dopamine clearance that does not rely on stimulated release, and that could measure clearance of higher concentrations of dopamine is desirable. Therefore, we developed an additional method using exogenously applied dopamine that could be used to quickly assess dDAT activity in existing dDAT mutants and that could reach concentrations closer to saturation.

A

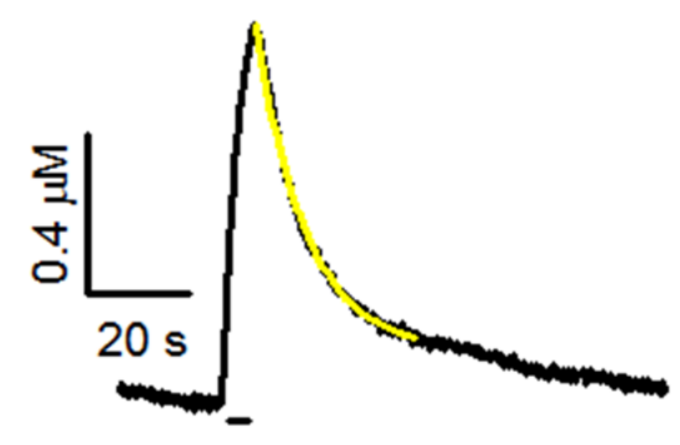

B

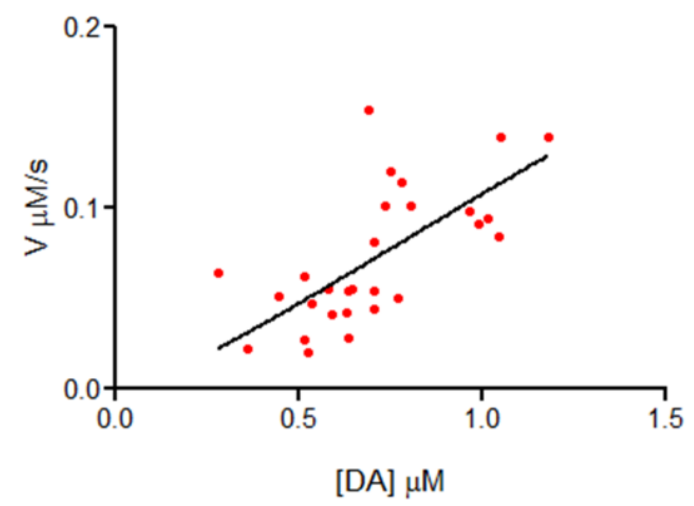

Figure 4.1: Modelling DAT with stimulated release. A) The decay portion of a 7s blue-light stimulation was fit with a first order exponential decay curve (yellow). The rate constant, $\mathrm{k}$, $\left(0.081 \mathrm{~s}^{-1}, \mathrm{r}^{2}=0.9959\right)$ is used to determine initial velocity, V. B) The initial velocity of dopamine clearance data (red circles) were fit to the Michaleis-Menten equation (line).

\subsubsection{Drosophila uptake via application of exogenous dopamine}

A carbon fiber microelectrode was implanted into the neuropil region of a homozygous UAS-ChR2;th-GAL4 Drosophila larval CNS. A capillary micropipette was 
backfilled with varying concentrations of dopamine and simultaneously implanted into the neuropil approximately $15-20 \mu \mathrm{m}$ from the electrode (Figure $4.2 \mathrm{~A}$ ). An additional horizontal cut was made on the posterior of the CNS to facilitate capillary implantation. Small volumes (pL) of dopamine were pressure-ejected into the neuropil and dopamine clearance observed. Figure 1B shows the concentration versus time profile for a $50 \mathrm{~ms}$ application of $210 \mathrm{pL}$ of $25 \mu \mathrm{M}$ dopamine pressure-ejected (arrow) into the neuropil. The cyclic voltammogram is used to confirm that the change in current is from dopamine. Due to the close distance between the electrode and pipette, dopamine is detected during the onset of pressure ejection. The difference between the amount of dopamine applied and detected is due primarily to uptake, but diffusion also plays a role. Figure $4.2 \mathrm{C}$ shows the concentration versus time profile for the pressure-ejection of varying amounts of dopamine in a single CNS. As concentration increases, so does the maximal dopamine concentration $[\mathrm{DA}]_{\max }$. The time course of signal decay from $[\mathrm{DA}]_{\max }$ can be used characterize dopamine clearance.

Picoliter volumes $(<1 \mathrm{~nL})$ of dopamine were applied and the signal decay from $68(\mathrm{n}=18)$ current vs time plots was fitted with a first-order exponential decay function (Equation 4.1), and the rate constant, $\mathrm{k}$, was calculated $\left(\mathrm{r}^{2} \geq 0.98\right)$. From this, initial velocity, $\mathrm{V}$, was determined (Equation 4.2), was plotted against $[\mathrm{DA}]_{\max }$, and fit to the Michaelis-Menten equation (Equation 4.3). Figure 4.2D shows the graph of both exogenous clearance (black squares) and stimulated release (red circles). The thatched green line indicates the fitted curve. $V_{\max }$ is estimated to be $0.35 \pm 0.04 \mu \mathrm{M} / \mathrm{s}$ and $\mathrm{K}_{\mathrm{m}}$ is estimated to be $4.5 \pm 0.4 \mu \mathrm{M}\left(\mathrm{r}^{2}=0.65\right)$. One problem with these estimates, however, is 
that they do not take into account diffusion. Although the distance between the spritzing pipette and electrode is small $(15-20 \mu \mathrm{m})$, when dopamine is applied, it can be cleared from the electrode by either uptake or diffusion. Without taking into account diffusion in intact tissue, $\mathrm{K}_{\mathrm{m}}$ values can be distorted, and affinity will appear higher (18). Therefore, to try to estimate the contribution of diffusion to these data, we tested fumin mutants, which lack a functional dDAT.

A

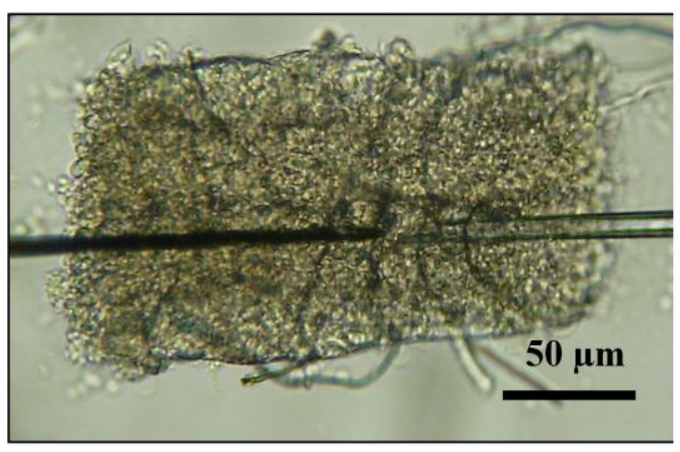

B

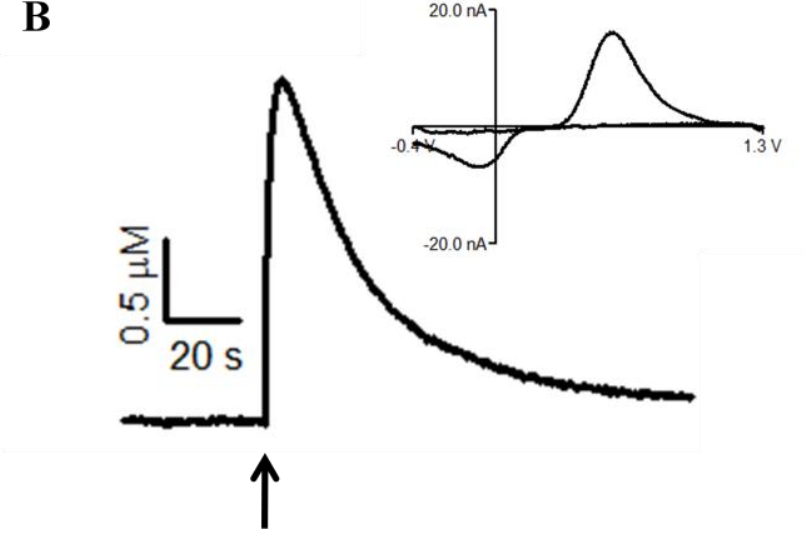

C

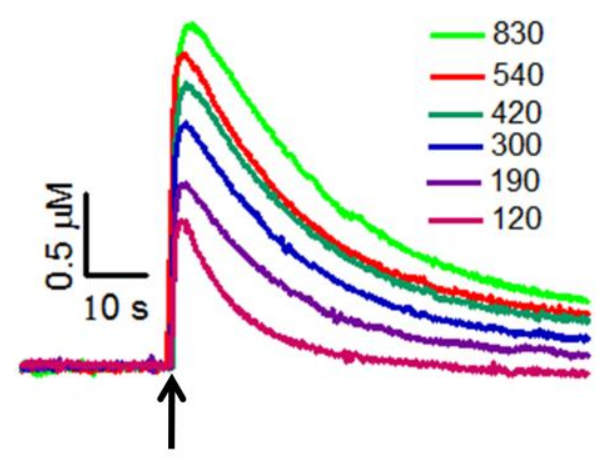

D

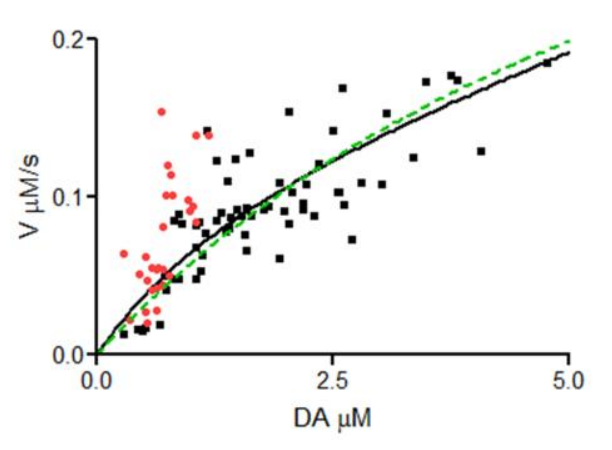

Figure 4.2: Application of exogenous dopamine in a Drosophila CNS. A) A carbon-fiber microelectrode and picospritzing capillary were simultaneously implanted approximately 15-20 $\mu \mathrm{M}$ apart in the neuropil of a Drosophila CNS. B) A picoliter (210 pL) volume of a $25 \mu \mathrm{M}$ dopamine solution was "spritzed" (arrow) into the tissue and .dopamine detected (CV inset). C) Representative trace of different volumes of exogenously applied dopamine in a single CNS. D) Initial velocity versus dopamine concentration (black squares) were fit with the Michaelis-Menten equation (green thatched line) and then corrected for diffusion (solid black line). Data from stimulated release is also shown (red circles). 


\subsubsection{Drosophila uptake in fumin mutants}

Fumin (fmn) mutants have abnormally high levels of activity and reduced rest (10). Fmn mutants have been used to determine that dopamine plays a role in temperature regulation in Drosophila (19); however, real-time assessment of dopamine clearance in fmn has been limited. Makos et al. observed that clearance of applied dopamine in fmn adults was decreased, but clearance in larvae has not yet been reported. Thus, dopamine uptake in a larval fmn mutants was investigated. Figure 4.3A shows the clearance of a similar concentration of detected dopamine in a larval fmn mutant compared to a UAS-ChR2; th-GAL4 larva. There is a clear decrease in the rate of clearance. Interestingly, when comparing amount of dopamine applied, rather than concentration detected, fmn mutants exhibit increased peak height. In Figure 4.3B, the same amount of dopamine (approx. 1000 femtomol) is applied to both fmn and UASChR2; th-GAL4, resulting in an almost 7-fold increase in peak height. The dramatic increase in peak height is likely because no dopamine is cleared by uptake. In DAT knockout mice, 5 -fold increases in extracellular concentration of dopamine have been reported (20). Increases in peak height following dopamine transporter inhibition have been reported in mammalian brain slice experiments $(4,21)$ and Drosophila larvae (13). Thus, it is not surprising that peak height is also increased in fmn.

One-phase exponential decay has been used to determine the rate constant of dopamine clearance in DAT KO mice (21). Similarly, 21 decay curves from fmn mutants $(n=6)$ were fit with a first-order decay function $\left(r^{2} \geq 0.98\right)$. Figure $4.3 C$ shows that there is a significant decrease in $\mathrm{k}$ for $f m n$ mutant flies (unpaired $\mathrm{t}$-test, $\mathrm{p}<0.0001$ ). The average 
rate constant for UAS-ChR2; th-GAL4 larvae is $0.062 \pm 0.004 \mathrm{~s}^{-1}$ whereas the average rate constant for $f m n$ larvae is $0.020 \pm 0.002 \mathrm{~s}^{-1}$. These results indicate that this method can be used to assess dDAT activity.

Data obtained from fmn mutants was used to correct $\mathrm{V}_{\max }$ and $\mathrm{K}_{\mathrm{m}}$ values for clearance not due to dDAT. The total rate of clearance depends on uptake and diffusion. Although some dopamine may be cleared by other transporters, such as the serotonin transporter (SERT), which has been shown to transport dopamine (9). Metabolism may also play a role; however, the time-scale for metabolic processes is longer, on the order of minutes (22), and monoamine oxidase, the enzyme responsible for metabolizing dopamine in mammals has not been identified in Drosophila. Because fmn lack a functional transporter, observed dopamine clearance at the electrode is assumed to be primarily due to diffusion. The average rate of decay from fmn mutants $\left(0.020 \pm 0.002 \mathrm{~s}^{-}\right.$ ${ }^{1}$ ) was used to correct the fitting parameters (Equation 4.3) used in our initial velocity versus dopamine concentration plot. Equation 4.3 now becomes: $V=\frac{V_{\text {max }} \bullet[\mathrm{DA}]_{\max }}{K_{m}+[\mathrm{DA}]_{\max }}+0.02 \mathrm{~s}^{-1} \bullet[\mathrm{DA}]_{\max }$ Equation 4.4 Correcting for diffusion, $\mathrm{V}_{\max }$ was estimated to be $0.13 \pm 0.1 \mu \mathrm{M} / \mathrm{s}$ and $\mathrm{K}_{\mathrm{m}}$ was estimated to be $1.7 \pm 0.8 \mu \mathrm{M}\left(\mathrm{r}^{2}=0.72\right)$. Figure $4.2 \mathrm{D}$ shows the curve-fit that has been corrected for diffusion (black). Although both curve-fits appear similar, the values for $\mathrm{V}_{\max }$ and $\mathrm{K}_{\mathrm{m}}$ are approximately 3-fold different. Without taking diffusion into account, DAT appears to have a lower affinity for dopamine $(4.5 \pm 0.4 \mu \mathrm{M}$ versus $1.7 \pm 0.8 \mu \mathrm{M})$. Similar distortions due to diffusion have been reported in chopped versus intact tissue (18). 
Our corrected $\mathrm{K}_{\mathrm{m}}$ is consistent with measurements of dDAT activity in transfected cells (9). Porzgen et al. reported a transporter affinity of $4.8 \pm 0.4 \mu \mathrm{M}$, which is in fair agreement with our reported value of $1.7 \pm 0.8 \mu \mathrm{M}$. They also report a $\mathrm{V}_{\max }=1.4 \mu \mathrm{M} / \mathrm{s}$, which is 10 times larger than our measurement of $0.13 \pm 0.1 \mu \mathrm{M} / \mathrm{s} . \mathrm{V}_{\max }$ is dependent upon cell densities, and there are likely to be differences in $\mathrm{V}_{\max }$ in a cell culture versus intact tissue. Therefore, this discrepancy is not surprising. Regardless of DAT density, transporter affinity should remain the same, making our overall results consistent with the only other kinetic estimates of dDAT. Furthermore, Porzgen et al. report a binding affinity of human DAT in transfected cells as $3.7 \pm 1.1 \mu \mathrm{M}$, which is in good agreement with other reported dopamine $K_{m}$ values found in mammalian studies $(9,16,23,24)$. A similar binding affinity in both hDAT and dDAT suggests that dDAT can be used to investigate human diseases involving dopamine dysregulation in a Drosophila model.
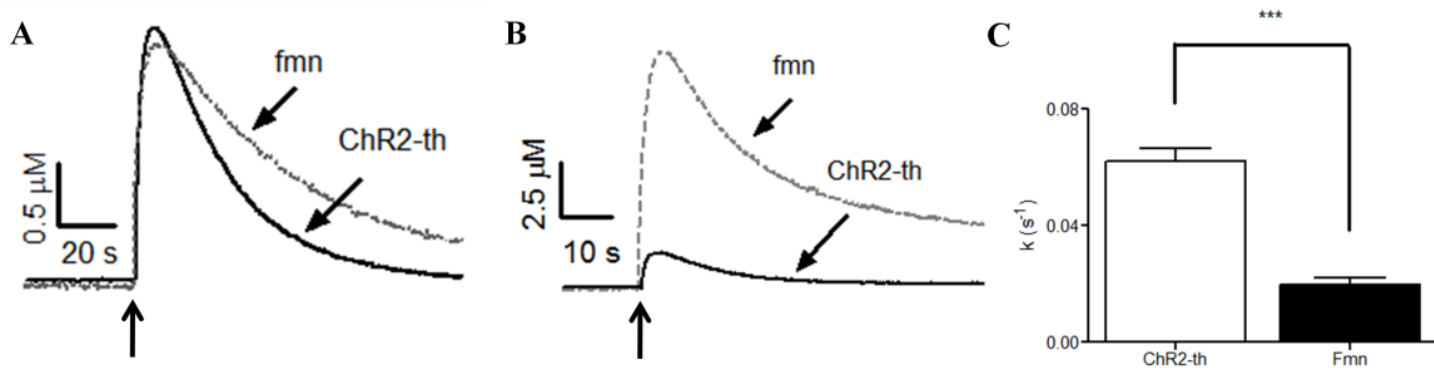

Figure 4.3: Application of exogenous dopamine in a fmn mutant. A) Concentration versus time profile for a fmn larva compared to a UAS-ChR2;th-GAL4 larva. For a similar concentration of dopamine detected, clearance in fmn is decreased. B) Peak height is larger in fmn compared to UAS-ChR2;thGAL4 when the same amount of dopamine is applied (approx. 1000 femtomoles). C) Comparison of the rate constant, $\mathrm{k}$, of UAS-ChR2;th-GAL4 $(\mathrm{n}=60)$ and fmn $(\mathrm{n}=21)$ larvae. Fmn mutants have significantly slower decay (unpaired t-test, $\mathrm{p}<0.0001$ ). 


\subsubsection{Drosophila uptake in the presence of cocaine}

We have previously shown that cocaine, a known DAT inhibitor, significantly prolongs blue-light evoked dopaminergic signaling in the CNS of Drosophila larvae (13). Similar results were found when exogenous dopamine was applied to the brain of Drosophila adults in the presence of cocaine (15). Here, we use our picospritzing method to investigate the effect of cocaine on the larval CNS, and calculate the first apparent affinity for dDAT in the presence of cocaine in intact tissue.

The CNS was incubated in $50 \mu \mathrm{M}$ cocaine for 15 minutes before exogenous dopamine was applied $(13,17)$. Figure $4.4 \mathrm{~A}$ shows example traces of dopamine after uptake inhibition. The concentration of dopamine that is detected is similar in the presence of cocaine compared to buffer, but the signal takes longer to return to baseline after cocaine. The signal decay for 9 curves $(n=3)$ was fit with a first-order exponential decay function and the average rate constant was $0.026 \mathrm{~s}^{-1}$, which is significantly slower than buffer alone (unpaired t-test, $\mathrm{p}<0.01$, Figure 4.4B).

In order to estimate the apparent affinity of DAT in the presence of cocaine, we used the rate constant to calculate initial velocity, and plotted $\mathrm{V}$ versus $[\mathrm{DA}]_{\max }$. We constrained $\mathrm{V}_{\max }$ to $0.13 \mu \mathrm{M} / \mathrm{s}$ and fit the plot to Equation 4.4 to correct for diffusion (Figure 4.4C). Cocaine is a competitive inhibitor of DAT (25). In the presence of a competitive inhibitor less dopamine is able to bind to DAT. The observed affinity, $\mathrm{K}_{\mathrm{m}, \mathrm{obs}}$, of DAT with cocaine is $8.8 \pm 3.0 \mu \mathrm{M}$, which is approximately 5 times higher than compared to buffer alone (Figure 4.4C). 
A

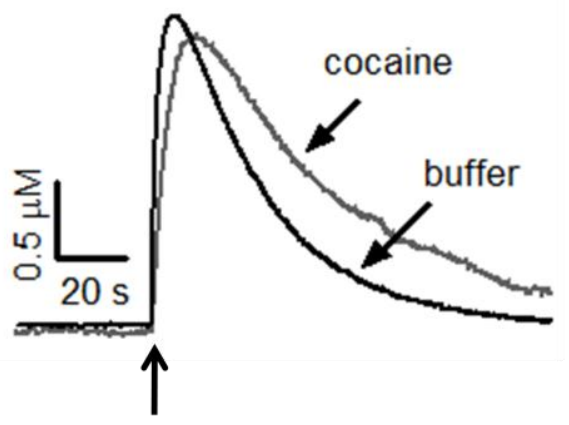

C

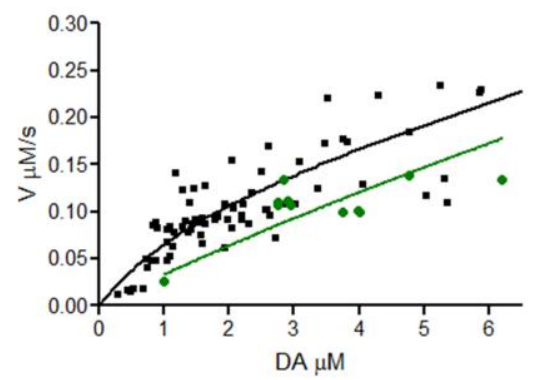

B

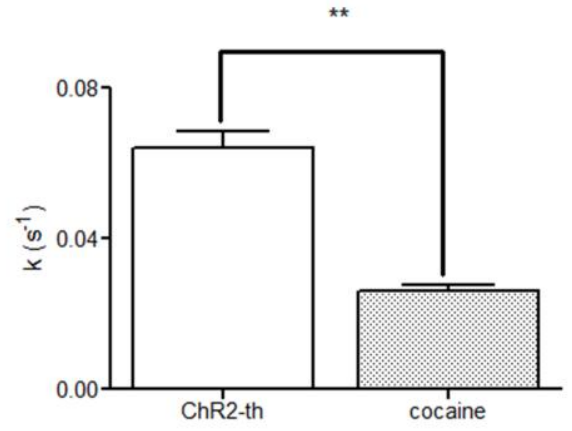

D

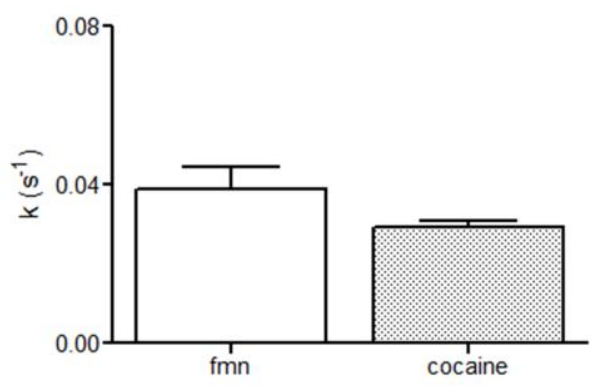

Figure 4.4: Application of exogenous dopamine in the presence of cocaine. A) Concentration versus time profile for the application of dopamine in a larva exposed to cocaine compared to buffer alone. For a similar concentration detected, clearance in the presence of cocaine is slowed. B) Comparison of the rate constant, $k$, of UAS-ChR2; th-GAL4 $(n=60)$ in buffer versus cocaine $(n=9)$. Larvae exposed to cocaine have significantly slower decay (unpaired t-test, $p<0.001$ ). C) Velocity versus concentration plot comparing uptake in the presence of cocaine (green) versus buffer (black). The observed affinity increases in the presence of a competitive inhibitor $(\mathrm{Km}, \mathrm{obs}=8.8 \pm 0.2 \mu \mathrm{M})$. D) Dopamine clearance in fmn mutants with and without $50 \mu \mathrm{M}$ cocaine. In the presence of cocaine the overall rate of decay does not change significantly $(\mathrm{n}=3$, paired t-test, $\mathrm{p}=0.2127)$.

Although cocaine has traditionally been used to study DAT in mammals, in

Drosophila cocaine has a higher affinity for the serotonin transporter (dSERT) than

dDAT (464 and 2,660 nM, respectively) (9). In addition, dSERT has been shown to have

a limited ability to transport dopamine, but has not been widely studied (9). In mammals, transport of neurotransmitters by other cell types has been reported, including dopamine by SERT $(26,27)$. To assess this effect in Drosophila, we first applied dopamine into the neuropil of a fmn mutant, which lacks a functional DAT, then incubated the CNS in 50 
$\mu \mathrm{M}$ cocaine to inhibit dSERT (17). The same volume of dopamine was applied to the neuropil in the presence of cocaine. Cocaine did not significantly alter the rate constant (Figure 4.4D, $\mathrm{n}=3$, paired t-test, $\mathrm{p}=0.2127$ ). We also compared the rate constants from UAS-ChR2;th-GAL4 with cocaine $(\mathrm{n}=9)$ to $f m n(\mathrm{n}=21)$ and found no significant difference (unpaired t-test, $\mathrm{p}=0.0869$ ). These results indicate that dSERT is not contributing significantly to dopamine uptake.

\subsubsection{Uptake in Drosophila mutants}

To assess whether our method can be used to as a screening tool for Drosophila mutants, we tested dopamine deficient and overexpressed DAT larvae. A line of dopamine deficient flies (DTHg ${ }^{F \pm} ;$ ple $)$ has recently been generated that lack tyrosine hydroxylase, the rate limiting enzyme in dopamine synthesis in neuronal cells, but still maintains tyrosine hydrozylase in non-neuronal cells (28). These flies show reduced activity, extended sleep time, locomotor deficits, and altered feeding patterns similar to those observed in dopamine deficient mice $(28,29)$. Although these flies lack dopamine, the overall morphology of the dopaminergic system remains intact. To test whether dDAT functions normally in these flies, we compared the rate of decay of exogenously applied dopamine in dopamine-deficient mutants. Figure 4.5A shows a concentration versus time profile for a similar amount of dopamine detected in dopamine deficient compared to UAS-ChR2;th-GAL4 larvae. The initial velocity of dopamine clearance is strikingly similar, although the curves deviate close to the baseline. In FSCV it is common that a complete return to baseline is not observed for every signal decay (13, 
30). Fitting the rate constant function from the time the signal ended until $80 \%$ of the signal decays helps account for baseline variance. The decay rate of dopamine-deficient larvae is $0.061 \pm 0.004 \mathrm{~s}^{-1}(\mathrm{n}=5)$, which is not significantly different than UAS-ChR2;thGAL4 (Figure 4C, unpaired t-test, $\mathrm{p}=0.8175$ ).

A

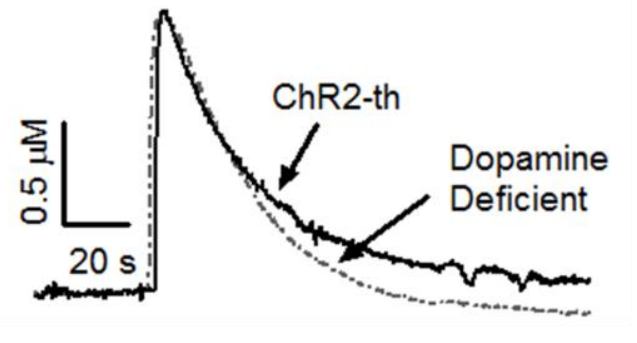

B

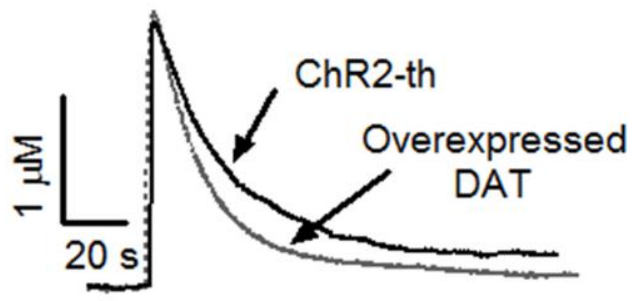

C

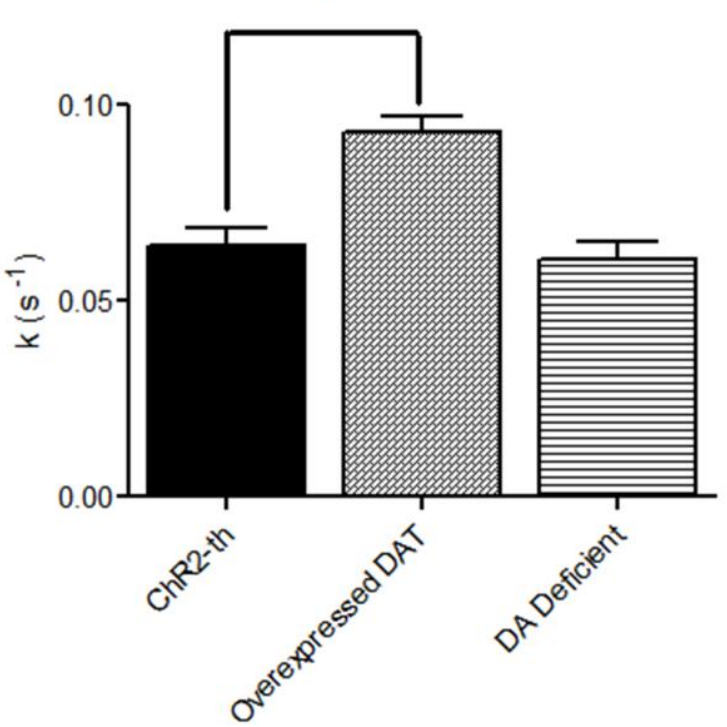

Figure 4.5: Signal decay in Drosophila mutants. A) Concentration versus time profile for the application of dopamine in a dopamine deficient larva compared to a UAS-ChR2;th-GAL4 larva. For a similar detected concentration, clearance is similar. B) Concentration versus time profile for overexpressed DAT compared to a UAS-ChR2;th-GAL4 larva. Clearance of dopamine is faster in overexpressed mutants. C) The decay rate of larvae overexpressing DAT is significantly faster than UAS-ChR2;th-GAL4 larvae (unpaired t-test, $\mathrm{p}=0.0291$ ). The decay rate of dopamine deficient larvae is not significantly different.

Our method was also tested on a newly generated Drosophila line overexpressing dDAT (NP2785;dDAT), which have dDAT overexpression in the population of neurons that become the posterior inferiorlateral protocerebrum (PPL1) cluster in adult Drosophila (31). The PPL1 neurons have projections throughout the neuropil in adults, and in the larval CNS $(31,32)$. The function of PPL1 or other distinct dopaminergic 
neuron populations in Drosophila is not well characterized, but recent evidence suggests that PPL1 neurons are involved in aversive olfactory learning and appetitively reinforced learning $(32,33)$. However, the effect of overexpression of DAT in Drosophila has not been studied behaviorally or through real-time measurements of dopamine.

Figure 4.5B shows a concentration versus time plot of a similar amount of dopamine detected for overexpressed dDAT and UAS-ChR2;th-GAL4 larvae. The decay of the overexpressed dDAT mutant is faster, indicating faster clearance of dopamine. For pooled results, the overexpressed dDAT mutant has a decay rate of $0.093 \pm 0.004 \mathrm{~s}^{-1}(\mathrm{n}=$ 7), which is significantly faster than UAS-ChR2; th-GAL4 larvae (Figure 4.5C, unpaired ttest, $\mathrm{p}=0.0291$ ). Overexpression of DAT in mammals results in increased sensitivity to psychostimulants and increased impulsive behavior $(8,34)$. FSCV measurements in mice overexpressing DAT have established that dopamine uptake is faster in the striatum (35). Understanding how DAT overexpression affects dopamine homeostasis is important because increased levels of DAT are implicated in diseases such as ADHD. By characterizing overexpression of DAT in distinct neuron populations in Drosophila, we hope to better understand the function of these neurons, and the role they play in dopamine regulation. 


\subsection{Conclusion}

We have shown that we can use fast-scan cyclic voltammetry to measure clearance of exogenously applied dopamine by the Drosophila dopamine transporter, and have provided the first $\mathrm{V}_{\max }$ and $\mathrm{K}_{\mathrm{m}}$ estimates for the dopamine transporter in an intact Drosophila CNS. Our reported transporter affinity is consistent with previously reported values of dDAT in transfected cells, and is homologous to human DAT affinity. The similarity of real-time dopaminergic signaling in Drosophila and mammals not only validates Drosophila as a model system for studying DAT, but also could reduce the use of higher order animals. Modeling clearance of exogenously applied dopamine allows a rapid method to screen transporter activity in Drosophila genetic mutants as well as studying disease models in Drosophila. Rapid screening of genetic mutants could help identify the genetic elements that are critical for DAT regulation of dopamine, and will lead to an increased understanding of dopamine homeostasis. 


\subsection{Experimental Methods}

\subsubsection{Chemicals}

Chemicals were purchased by Sigma-Aldrich (St. Louis, MO) and used as received unless otherwise specified. Solutions were made using Milli-Q water (Millipore, Billerica, MA). All electrode calibrations, drug solutions and Drosophila preparations were made using a modified Schneider's buffer $\left(15.2 \mathrm{mM} \mathrm{MgSO}_{4}, 21 \mathrm{mM} \mathrm{KCl}, 3.3\right.$ $\mathrm{KH}_{2} \mathrm{PO}_{4}, 36 \mathrm{mM} \mathrm{NaCl}, 5.8 \mathrm{mM} \mathrm{NaH}_{2} \mathrm{PO}_{4}, 5.4 \mathrm{mM} \mathrm{CaCl}_{2}, 11.1 \mathrm{mM}$ glucose, $5.3 \mathrm{mM}$ trehalose, $\mathrm{pH}$ 6.2). Larval food for stimulated release experiments was prepared with 10 $\mathrm{mM}$ all trans-retinal mixed with yeast and water.

\subsection{2: Electrochemical Measurements}

Carbon-fiber microelectrodes were constructed by aspirating individual T-650 carbon fibers (Cytec Engineering Materials, West Patterson, NJ) into a $1.2 \mathrm{~mm} \mathrm{X} 0.68$ mm glass capillary (A-M Systems, Carlsburg, WA). Electrodes were formed by pulling the capillary with a vertical pipette puller (Narishige PE-21, East Meadow, NY). The length of the carbon fiber was trimmed to $40-60 \mu \mathrm{m}$ with a scapel. To seal the fiber-glass interface, electrodes were expoxied with Epon Resin 828 (Miller-Stephenson, Danbury, CT) mixed with 14\% (w/w) m-phenylenediamine hardener (Fluka, Milwaukee, WI). After curing in an oven at $100{ }^{\circ} \mathrm{C}$ for $2 \mathrm{~h}$ followed by $150{ }^{\circ} \mathrm{C}$ overnight, the electrodes were soaked in isopropanol and backfilled with $1 \mathrm{M} \mathrm{KCl}$ before use. 
A Dagan Chem-Clamp potentiostat (Dagan, Minneapolis, MN; custom modified) and Tar Heel CV software (gift of Mark Wightman, University of North Carolina) were used to collect data. The cyclic voltammogram waveform was generated and applied by a homemade breakout box with PCI 6052 and 6711 boards (National Instruments, Austin, TX). The electrode was scanned from -0.4 to $1.3 \mathrm{~V}$ and back at a scan rate of $400 \mathrm{~V} / \mathrm{s}$. The repetition rate for all data collected was $10 \mathrm{~Hz}$ with the exception of $\mathrm{DTHg}^{\mathrm{FS}}$; ple larvae and calibrations, which were collected at $60 \mathrm{~Hz}$. $\mathrm{An} \mathrm{Ag} / \mathrm{AgCl}$ reference electrode was placed in the Petri dish near the ventral nerve cord. Electrodes were allowed to cycle in buffer for 15 min prior to implantation and calibrated with a $1.0 \mu \mathrm{M}$ dopamine solution before and after use in situ. For cocaine experiments, a second calibration was performed in the presence of drug to account for possible drug effects on the electrode sensitivity.

Picospritzing capillaries were made by pulling a $1.2 \mathrm{~mm}$ X $0.68 \mathrm{~mm}$ glass capillary (A-M Systems, Carlsburg, WA) with a vertical pipette puller (Narishige PE-21, East Meadow, NY). The capillary surface was polished on a beveling wheel (K.T. Brown Type; Sutter Instrument Co. Model BV-10, Novoto, CA) at an angle of $30^{\circ}$. Capillaries were back filled with dopamine solutions ranging from 10-100 $\mu \mathrm{M}$. Dopamine solution was pressure ejected using a Picospritzer III (Parker Hannifin, Fairfield, NJ). Each pipette was calibrated by ejecting dopamine solution into vegetable oil, measuring the radius of the ejected droplet with a reticle, and calculating the volume $\left(\right.$ volume $\left.=4 / 3 \pi r^{3}\right)$.

\subsubsection{Preparation of the Drosophila CNS}


Flies containing UAS-ChR2 (Bloomington Stock Center, Bloomington, IN) were crossed to flies expressing th-GAL4 (a gift from Jay Hirsh, University of Virginia) to generate homozygous lines with a th-GAL4;UAS-ChR2 genotype. $F m n, \mathrm{DTHg}^{\mathrm{FS}}$ GAL4;UAS- ple, and NP2785-GAL4;UAS-dDAT flies were a gift from Jay Hirsh, University of Virginia. For stimulated release experiments, 3-day-old L3W larvae were allowed to feed on a mixture of all-trans retinal mixture in the dark for two days prior to experimentation. For all experiments, 5-day-old L3W larvae were dissected in a modified Schneider's buffer. The CNS was dissected-out and optic lobes removed by making a horizontal cut in the anterior-most portion of the ventral nerve cord. For picospritzing experiments, an additional horizontal cut was made at the posterior-most portion of the ventral nerve cord. Isolated ventral nerve cords were adhered neuropil side down in a Petri dish (Becton Dickinson, Franklin Lakes, NJ) with $3 \mathrm{~mL}$ of buffer. For all experiments an electrode was inserted with a micromanipulator (Siskiyou, Grants Pass, OR) into the neuropil using the 40x water immersion lens on a Axio Examiner microscope (Carl Zeiss, Thornwood, New York). The electrode was inserted 4-6 segments away from the cut edge. For picospritzing experiments, the picospritzing capillary was inserted $15-20 \mu \mathrm{m}$ away from the electrode. The electrode and capillary were allowed to equilibrate after implantation for $5 \mathrm{~min}$ prior to data collection.

\subsection{4: Data Analysis}

Data were analyzed using GraphPad Prism (GraphPad Software, San Diego, CA). Unpaired t-tests were used to compare rates of decay for pharmacological and mutant 
experiments, while paired t-tests were used to compare decay rate or stimulated release within the same animal. Data were considered different at a 95\% confidence level. Error bars are standard error of the mean. All single exponential decay functions were fit with Graphpad and had an $r^{2} \geq 0.98$. Non-linear regression analysis was also done with Graphpad. 


\subsection{References:}

1. Giros, B., Jaber, M., Jones, S. R., Wightman, R. M., and Caron, M. G. (1996) Hyperlocomotion and indifference to cocaine and amphetamine in mice lacking the dopamine transporter, Nature 379, 606-612.

2. Kuhar, M. J., Ritz, M. C., and Boja, J. W. (1991) The dopamine hypothesis of the reinforcing properties of cocaine, Trends in Neurosciences 14, 299-302.

3. John, C. E., and Jones, S. R. (2007) Voltammetric characterization of the effect of monoamine uptake inhibitors and releasers on dopamine and serotonin uptake in mouse caudate-putamen and substantia nigra slices, Neuropharmacology 52, 1596-1605.

4. Jones, S. R., Garris, P. A., and Wightman, R. M. (1995) Different effects of cocaine and nomifensine on dopamine uptake in the caudate-putamen and nucleus accumbens., J. Pharmacol. Exp. Ther. 274, $396-403$.

5. Cass, W. A., Zahniser, N. R., Flach, K. A., and Gerhardt, G. A. (1993) Clearance of Exogenous Dopamine in Rat Dorsal Striatum and Nucleus Accumbens: Role of Metabolism and Effects of Locally Applied Uptake Inhibitors, Journal of Neurochemistry 61, 2269-2278.

6. Cragg, S. J., Nicholson, C., Kume-Kick, J., Tao, L., and Rice, M. E. (2001) Dopamine-Mediated Volume Transmission in Midbrain Is Regulated by Distinct Extracellular Geometry and Uptake, Journal of Neurophysiology 85, $1761-1771$.

7. Spielewoy, C., Roubert, C., Hamon, M., Nosten-Bertrand, M., Betancur, C., and Giros, B. (2000) Behavioural disturbances associated with hyperdopaminergia in dopamine-transporter knockout mice., Behavioural Pharmacology 11, 279-290.

8. Salahpour, A., Ramsey, A. J., Medvedev, I. O., Kile, B., Sotnikova, T. D., Holmstrand, E., Ghisi, V., Nicholls, P. J., Wong, L., Murphy, K., Sesack, S. R., Wightman, R. M., Gainetdinov, R. R., and Caron, M. G. (2008) Increased amphetamine-induced hyperactivity and reward in mice overexpressing the dopamine transporter, P. Natl. Acad. Sci. USA 105, $4405-4410$.

9. Porzgen, P., Park, S. K., Hirsh, J., Sonders, M. S., and Amara, S. G. (2001) The Antidepressant-Sensitive Dopamine Transporter inDrosophila melanogaster: A Primordial Carrier for Catecholamines, Mol. Pharmacol. 59, 83-95.

10. Kume, K., Kume, S., Park, S. K., Hirsh, J., and Jackson, F. R. (2005) Dopamine Is a Regulator of Arousal in the Fruit Fly, The Journal of Neuroscience 25, 7377 7384. 
11. Wisor, J. P., Nishino, S., Sora, I., Uhl, G. H., Mignot, E., and Edgar, D. M. (2001) Dopaminergic Role in Stimulant-Induced Wakefulness, The Journal of Neuroscience 21, $1787-1794$.

12. Makos, M. A., Kim, Y.-C., Han, K.-A., Heien, M. L., and Ewing, A. G. (2009) In Vivo Electrochemical Measurements of Exogenously Applied Dopamine in Drosophila melanogaster, Anal. Chem. 81, 1848-1854.

13. Vickrey, T. L., Condron, B., and Venton, B. J. (2009) Detection of Endogenous Dopamine Changes in Drosophila melanogaster Using Fast-Scan Cyclic Voltammetry, Anal. Chem. 81, 9306-9313.

14. Vickrey, T. L., and Venton, B. J. (2011) Drosophila Dopamine2-like Receptors Function as Autoreceptors, ACS Chem. Neurosci. 2, 723-729.

15. Makos, M. A., Han, K.-A., Heien, M. L., and Ewing, A. G. (2009) Using in Vivo Electrochemistry To Study the Physiological Effects of Cocaine and Other Stimulants on the Drosophila melanogaster Dopamine Transporter, ACS Chem. Neurosci. 1, 74-83.

16. Sabeti, J., Adams, C. E., Burmeister, J., Gerhardt, G. A., and Zahniser, N. R. (2002) Kinetic analysis of striatal clearance of exogenous dopamine recorded by chronoamperometry in freely-moving rats, J. Neurosci. Methods 121, 41-52.

17. Borue, X., Cooper, S., Hirsh, J., Condron, B., and Venton, B. J. (2009) Quantitative evaluation of serotonin release and clearance in Drosophila, J. Neurosci. Methods 179, 300-308.

18. Near, J. A., Bigelow, J. C., and Wightman, R. M. (1988) Comparison of uptake of dopamine in rat striatal chopped tissue and synaptosomes., Journal of Pharmacology and Experimental Therapeutics 245, 921 -927.

19. Ueno, T., Tomita, J., Kume, S., and Kume, K. (2012) Dopamine Modulates Metabolic Rate and Temperature Sensitivity in Drosophila melanogaster, PLoS ONE 7, e31513.

20. Jones, S. R., Gainetdinov, R. R., Jaber, M., Giros, B., Wightman, R. M., and Caron, M. G. (1998) Profound neuronal plasticity in response to inactivation of the dopamine transporter, Proceedings of the National Academy of Sciences 95, $4029-4034$.

21. John, C. E., Budygin, E. A., Mateo, Y., and Jones, S. R. (2006) Neurochemical characterization of the release and uptake of dopamine in ventral tegmental area 
and serotonin in substantia nigra of the mouse, Journal of Neurochemistry 96, 267-282.

22. O’Neill, R. D. (1994) Microvoltammetric techniques and sensors for monitoring neurochemical dynamics in vivo. A review, Analyst 119, 767-779.

23. Hebert, M. A., and Gerhardt, G. A. (1999) Age-Related Changes in the Capacity, Rate, and Modulation of Dopamine Uptake within the Striatum and Nucleus Accumbens of Fischer 344 Rats: An In Vivo Electrochemical Study, Journal of Pharmacology and Experimental Therapeutics 288, 879 -887.

24. Zahniser, N. R., Larson, G. A., and Gerhardt, G. A. (1999) In vivo dopamine clearance rate in rat striatum: regulation by extracellular dopamine concentration and dopamine transporter inhibitors, J. Pharmacol. Exp. Ther. 289, 266-277.

25. Wu, Q., Reith, M. E. A., Kuhar, M. J., Carroll, F. I., and Garris, P. A. (2001) Preferential Increases in Nucleus Accumbens Dopamine after Systemic Cocaine Administration Are Caused by Unique Characteristics of Dopamine Neurotransmission, The Journal of Neuroscience 21, 6338 -6347.

26. Larsen, M. B., Sonders, M. S., Mortensen, O. V., Larson, G. A., Zahniser, N. R., and Amara, S. G. (2011) Dopamine Transport by the Serotonin Transporter: A Mechanistically Distinct Mode of Substrate Translocation, The Journal of Neuroscience 31, $6605-6615$.

27. Bunin, M. A., and Wightman, R. M. (1999) Paracrine neurotransmission in the CNS: involvement of 5-HT, Trends in Neurosciences 22, 377-382.

28. Riemensperger, T., Isabel, G., Coulom, H., Neuser, K., Seugnet, L., Kume, K., Iché-Torres, M., Cassar, M., Strauss, R., Preat, T., Hirsh, J., and Birman, S. (2011) Behavioral consequences of dopamine deficiency in the Drosophila central nervous system, Proceedings of the National Academy of Sciences 108, 834 -839.

29. Zhou, Q.-Y., and Palmiter, R. D. (1995) Dopamine-deficient mice are severely hypoactive, adipsic, and aphagic, Cell 83, 1197-1209.

30. Bath, B. D., Michael, D. J., Trafton, B. J., Joseph, J. D., Runnels, P. L., and Wightman, R. M. (2000) Subsecond Adsorption and Desorption of Dopamine at Carbon-Fiber Microelectrodes, Anal. Chem. 72, 5994-6002.

31. Hirsh, J. (2012) Unpublished data, University of Virginia. 
32. Mao, Z, and Davis, R. L. (2009) Eight Different Types of Dopaminergic Neurons Innervate the Drosophila Mushroom Body Neuropil: Anatomical and Physiological Heterogeneity, Frontiers in Neural Circuits 3.

33. Busto, G. U., Cervantes-Sandoval, I., and Davis, R. L. (2010) Olfactory Learning in Drosophila, Physiology 25, $338-346$.

34. Adriani, W., Boyer, F., Gioiosa, L., Macrì, S., Dreyer, J.-L., and Laviola, G. (2009) Increased impulsive behavior and risk proneness following lentivirusmediated dopamine transporter over-expression in rats' nucleus accumbens, Neuroscience 159, 47-58.

35. Kile, B. M., Walsh, P. L., McElligott, Z. A., Bucher, E. S., Guillot, T. S., Salahpour, A., Caron, M. G., and Wightman, R. M. (2012) Optimizing the Temporal Resolution of Fast-Scan Cyclic Voltammetry, ACS Chem. Neurosci. 3, 285-292. 


\section{Chapter 5}

\section{Conclusions and future directions}




\section{Chapter 5: Conclusions and Future Directions}

In this thesis, I have described methods using fast-scan cyclic voltammetry at carbon-fiber microelectrodes to measure dopaminergic signaling in Drosophila. This chapter summarizes the main conclusions and looks ahead to future studies in Drosophila that could be used to improve understanding of dopamine homeostasis and the role that dopamine plays in human diseases.

\section{1: Investigating real-time dopamine in Drosophila adults and in disease models}

In chapters 2 and 3 of this dissertation, I described a novel method to characterize real-time dopamine release in Drosophila larvae using optogenetics. This method provided the first real-time measurements of dopaminergic signaling in the fruit fly. I showed that dopamine release was vesicular in nature, and that the response to pharmacological agents, such as synthesis disrupters or DAT inhibitors, is consistent with mammalian studies. I was also able to prove that Drosophila D2 receptors function as autoreceptors, and have a similar response to dopamine receptor agonists and antagonists as mammalian receptors - a result that could not be achieved without real-time measurements. Previous investigations in Drosophila, such as genetic, behavioral and immunohistochemical studies, suggest that dopamine signaling is homologous to that of mammals, and my work was able to confirm this.

A logical next step in this research is to develop a method to characterize release in adults, so that dopaminerigic signaling across multiple stages of development can be 
studied. This would be useful particularly for understanding dopamine signaling across the life span in neurodegenerative disease models, such as Parkinson's. Parkinson's disease afflicts over 1.5 million people in the US alone, and typically is not diagnosed until approximately $80 \%$ of striatal dopamine (DA) and $30 \%$ of substantia nigra neurons have degenerated $(1,2)$. Due to dramatic neuron loss before diagnosis, treatment regimes are often ineffective and short-lasting and early therapeutic intervention is uncommon. Therefore, detecting early abnormalities in dopaminergic signaling could improve our understanding of the changes that may precede clinically diagnosable Parkinson's disease, and lead to the development of screens for pre-Parkinson's disease symptoms. I have completed some preliminary work on optical stimulation of dopamine in adults, which focused on immobilization of awake adults and microdissection of the skull cap. Figure 5.1A shows an awake adult immobilized in dental wax and skull cap removed. An electrode has been implanted into the brain (Figure 5.1B); however, a measurable dopamine signal was not produced (data not shown). Future work should focus on visualizing dopaminergic cell bodies in the adult brain with GFP before implantation to insure that the electrode is implanted into the correct area. In addition, in Figure 5.1B the brain tissue appears "pinched", which could be alleviated by relaxing the extracellular matrix in the brain with collagenase (3). 
A

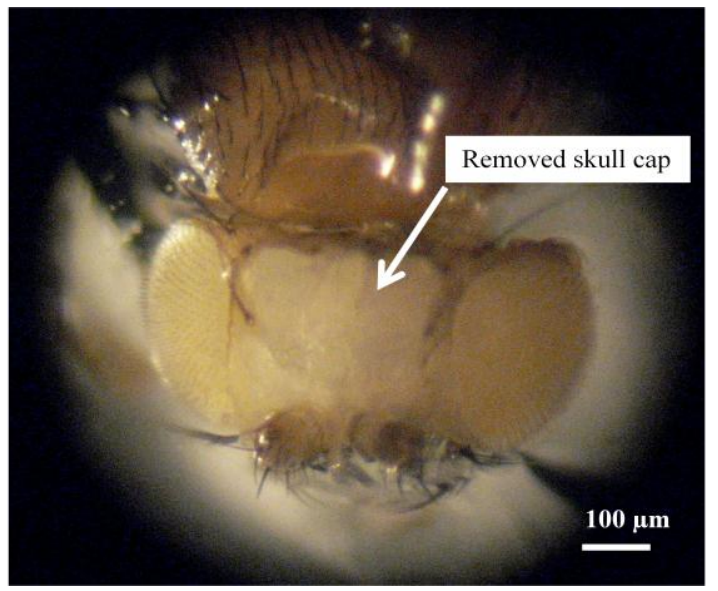

B

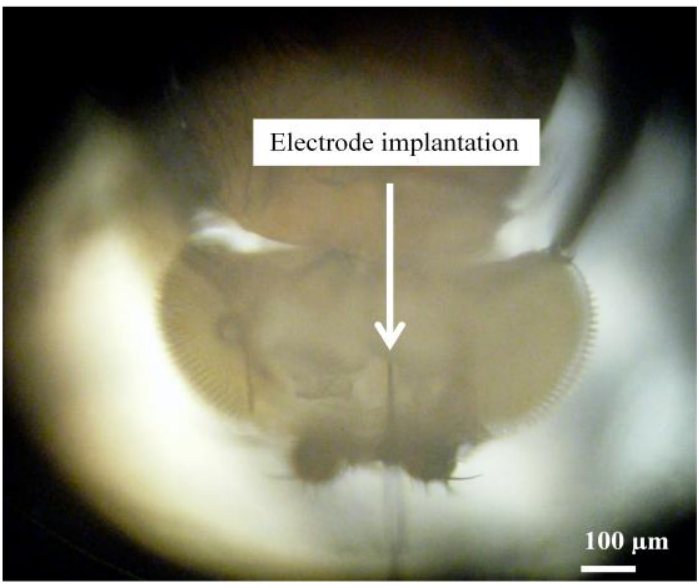

Figure 5.1: Immobilization (A) and electrode implantation (B) in adult Drosophila.

Once dopaminergic signaling is fully characterized in th-GAL4;UAS-ChR2 lines, additional flies could be bred that express both ChR2 and Parkinson's-like mutations in dopaminergic neurons using the Gal4/UAS system. The phenotypes of the currently known Parkinson's disease-associated genes have been well-characterized in Drosophila, including $\alpha$-synuclein, PINK1, and parkin, which are associated with familial Parkinson's disease (4). Alpha-synuclein is of interest because it is an abundant neuronal protein implicated in Parkinson's disease, but its function in disease etiology is unclear; in Drosophila it produces locomotor defects and adult-onset dopaminergic cell loss $(5,6)$. Parkin and PINK1 are of interest because they both are involved in mitochondrial recycling pathways; in Drosophila defects of these mitochondria-associated proteins usually lead to locomotor dysfunction, degenerated wing muscles, shortened life-span, and eventual dopaminergic cell death (7). Coupling the extensive behavioral and genetic results in these disease models with real-time dopaminergic signaling, would help elucidate early changes in signaling occurring before onset of classical Parkinson's 
disease symptoms and may provide a useful, simple model for studying preparkinsonism.

\section{2: Investigation of transporters and large scale screening methods}

In chapter 4 of this dissertation, I report a method to model clearance of exogenously applied dopamine, providing the first estimates of DAT kinetics in an intact CNS. To prove the utility of the method for screening mutant flies, dopamine clearance was compared in dopamine deficient flies and flies overexpressing DAT. Dopamine deficient flies have no significant changes in uptake, while flies overexpressing DAT have increased uptake. The ability to screen transporter mutants will help identify the genetic elements that are critical for DAT regulation of dopamine, and will lead to an increased understanding of dopamine homeostasis.

The next step of this research would be to complete a large scale screening of dDAT mutants. A variety of GAL4 drivers for distinct dopaminergic populations of neurons have been developed (8). Once Drosophila lines with DAT mutations in each of these populations are made (our collaborator, Jay Hirsh, has several of these mutant lines ready to test), our method can be used to assess differences in DAT activity. Not only will screening assist with understanding the functional nature of the dopaminergic populations, but also by testing flies with specific mutations of dDAT, the domains of the protein crucial for uptake to function will be indentified.

Another important extension of this method will be to investigate additional neurotransmitter transporters such as the serotonin transporter (SERT). SERT is of 
particular interest because polymorphisms in SERT in humans are linked to behavioral traits such as anxiety and depression as well as autism, eating disorders, and substance abuse (9). In addition, SERT is the target for the class of anti-depressants called SSRIs. The efficacy of SSRIs appears to be highly dependent on SERT polymorphisms (10-12). Therefore, a characterization of uptake mechanisms in Drosophila SERT mutants would allow us to determine the genetic components critical for SERT regulation of serotonin. Additional studies could be performed in the presence of SSRIs, and drug effects on serotonin homeostasis could be determined.

\section{3: Final Remarks}

Overall, this dissertation provides two useful methods that can be used to detect real-time dopamine signaling in Drosophila. These methods have the potential to provide an increased understanding of the basic neurobiology of dopamine regulation as well as human diseases. 


\section{4: Reference List}

1. Cleveland Clinic. Parkinson's Disease: Incidence.[Accessed online Feb 2011]

2. Feany, M. B., and Bender, W. W. (2000) A Drosophila model of Parkinson's disease, Nature 404, 394-398.

3. Makos, M. A., Kim, Y.-C., Han, K.-A., Heien, M. L., and Ewing, A. G. (2009) In Vivo Electrochemical Measurements of Exogenously Applied Dopamine in Drosophila melanogaster, Anal. Chem. 81, 1848-1854.

4. Guo, M. (2010) Chapter 1 - What have we learned from Drosophila models of Parkinson's disease? In Recent Advances in Parkinson'S Disease Translational and Clinical Research, pp 2-16, Elsevier.

5. Feany, M. B., and Bender, W. W. (2000) A Drosophila model of Parkinson's disease, Nature 404, 394-398.

6. Perez, R. G., and Hastings, T. G. (2004) Could a loss of $\alpha$-synuclein function put dopaminergic neurons at risk?, Journal of Neurochemistry 89, 1318-1324.

7. Yang, Y., Gehrke, S., Imai, Y., Huang, Z., Ouyang, Y., Wang, J.-W., Yang, L., Beal, M. F., Vogel, H., and Lu, B. (2006) Mitochondrial pathology and muscle and dopaminergic neuron degeneration caused by inactivation of Drosophila Pink1 is rescued by Parkin, Proceedings of the National Academy of Sciences 103, $10793-10798$.

8. Aso, Y., Siwanowicz, I., Bräcker, L., Ito, K., Kitamoto, T., and Tanimoto, H. (2010) Specific Dopaminergic Neurons for the Formation of Labile Aversive Memory, Current Biology 20, 1445-1451.

9. $\quad$ Lesch, K.-P., Bengel, D., Heils, A., Sabol, S. Z., Greenberg, B. D., Petri, S., Benjamin, J., Müller, C. R., Hamer, D. H., and Murphy, D. L. (1996) Association of Anxiety-Related Traits with a Polymorphism in the Serotonin Transporter Gene Regulatory Region, Science 274, $1527-1531$.

10. Cunningham, K. A., Bradberry, C. W., Chang, A. S., and Reith, M. E. A. (1995) The role of serotonin in the actions of psychostimulants: molecular and pharmacological analyses, Behavioural Brain Research 73, 93-102.

11. Ng, C. H., Easteal, S., Tan, S., Schweitzer, I., Ho, B. K. W., and Aziz, S. (2006) Serotonin transporter polymorphisms and clinical response to sertraline across ethnicities, Progress in Neuro-Psychopharmacology and Biological Psychiatry 30, 953-957. 
12. Serretti, A., Benedetti, F., Zanardi, R., and Smeraldi, E. (2005) The influence of Serotonin Transporter Promoter Polymorphism (SERTPR) and other polymorphisms of the serotonin pathway on the efficacy of antidepressant treatments, Progress in Neuro-Psychopharmacology and Biological Psychiatry 29, 1074-1084.

13. Kuhar, M. J., Ritz, M. C., and Boja, J. W. (1991) The dopamine hypothesis of the reinforcing properties of cocaine, Trends in Neurosciences 14, 299-302. 